\title{
Twisted differential cohomology
}

\author{
Ulrich Bunke*and Thomas Nikolaus ${ }^{\dagger}$
}

June 13, 2014

\section{Contents}

1 Introduction 2

1.1 On the contents of the paper . . . . . . . . . . . . 2

1.2 The case of complex $K$-theory $\ldots \ldots \ldots \ldots \ldots$

I Twisted cohomology via graded ring spectra 8

2 Graded ring spectra 8

3 Twisted cohomology 10

4 Twisted differential cohomology 16

5 Properties of twisted differential cohomology 22

II De Rham models 25

6 Twisted de Rham complexes 25

7 Uniqueness of differential refinements of twists 30

8 Existence of differential refinements of twists 33

III Examples 40

*NWF I - Mathematik, Universität Regensburg 93040 Regensburg, Germany, ulrich.bunke@mathematik.uni-regensburg.de

${ }^{\dagger}$ NWF I - Mathematik, Universität Regensburg 93040 Regensburg, Germany, thomas.nikolaus@mathematik.uni-regensburg.de 
10 From differential $\mathrm{bgl}_{1}$ to twists

11 Examples of twists

12 The twisted Atiyah-Hirzebruch spectral sequence 53

IV Appendix $\quad 57$

A Technical facts about graded ring spectra 57

B Locally constant sheaves $\quad 64$

\section{Introduction}

\subsection{On the contents of the paper}

The main goal of the present paper is the construction of twisted generalized differential cohomology theories and the comprehensive statement of its basic functorial properties. Technically it combines the homotopy theoretic approach to (untwisted) generalized differential cohomology developed in [HS05], BG13, Bun] with the $\infty$-categorical treatement of twisted cohomology in ABG11. One of the main motivations for twisted differential cohomology is the theory of integration (resp. pushforward) in non orientable situations. Our approach leads to such a theory, which will be discussed elsewhere.

Following an idea of the second author a neat way to state all the properties of a twisted generalized differential cohomology is to say that it forms a sheaf of graded ring spectra on the category of smooth manifolds where the underlying sheaf of gradings is the Picard stack of differential twists. This formulation incorporates the multiplicative structure of twisted cohomology (which adds twists), automorphisms of twists, Mayer-Vietoris sequences as well as the functoriality in the underlying manifold.

Given the approach of BG13] the construction of twisted generalized differential cohomology is actually easy once the differential twists are understood. So the main emphasis of the present paper is on the investigation of the Picard groupoid of differential twists. Our main results concern the existence of differential refinements of topological twists and the classification of such refinements. As an interesting aspect of the theory we show that for a commutative ring spectrum $R$ there is a map (anticipated e.g. in [DFM11]) from a version of differential $\operatorname{bgl}_{1}(R)$-cohomology to differential $R$-twists.

We now explain the structure of the paper in detail. It consists of three parts and a technical appendix. In the first part we develop twisted generalized differential cohomology in general. In the second part we consider in detail the differential form part of differential 
twists and discuss the existence and uniqueness of differential refinements of topological twists. The third part contains more concrete material and examples.

In the first part we develop the general theory of twisted generalized differential cohomology starting with the notion of a graded ring spectrum in Section 2. In Section 3 we review the homotopy theoretic version of twisted cohomology using the language of graded ring spectra. In Section 4 we introduce the Picard- $\infty$ stack of differential twists and construct the sheaf of graded ring spectra representing twisted generalized cohomology. The first part is concluded with Section 5 where we list explicitly some of the functorial structures of twisted differential cohomology.

In the second part we investigate differential twists in detail. While the definition of differential twists given in Section 4 is straightforward it is not at all obvious whether a given topological twist has a differential refinement at all, and how many of such refinements exist. In the preparatory Section 6 we introduce a particular class of Rham models which can conveniently be used in the construction of differential twists. In Section 7 we show under mild assumptions that the extension of a topological twist to a differential twist is unique up to non-canonical equivalence. The existence of differential twists is studied in Section 8 .

The third part is devoted to special cases and examples. While the general theory works for arbitrary commutative ring spectra all examples of interest satisfy a set of particular assumptions termed differentially simple in Section 9. This assumption simplifies the choice of suitable de Rham models considerably. In Section 10 we show that cycles for differential $\operatorname{bgl}_{1}(R)$-cohomology determine differential $R$-twists. This provides one explicit way to construct differential twists. In this framework we discuss, in particular, the construction of differential twists from higher geometric gerbes. In Section 12 we show how one can determine the de Rham part of the differential twist refining a topological twist $E$ from the knowledge of differentials of the Atiyah-Hirzebruch spectral sequence associated to $E$.

In the second subsection of this introduction we explain some new contributions of this paper in the example of complex $K$-theory.

\subsection{The case of complex $K$-theory}

The study of field theories involving higher-degree differential forms on manifolds with $B$ field backgrounds lead to quantization conditions which could conveniently be explained by the requirement, that the field strength represents the Chern character of a twisted $K$-theory class. Subsequently various models of twisted $K$-theory have been constructed. It was clear from the beginning, that twisted $K$-theory fixes the topological background, and the actual fields are classified by twisted differential $K$-theory classes. It turned out to be important to understand the twists themselves in the framework of differential cohomology. For a detailed description of the phyiscal motivation we refer to [DFM11]. Twisted complex $K$-theory and its differential version have been investigated to some extent [DK70], [TXLG04], [AS06], [AS04], [FHT13], [FHT11a], [FHT11b], [BV13], [BCM ${ }^{+} 02$ ], 
Gom13], Har12, [MP07, [HM12] (we refrain from listing paper dealing with non-twisted differential $K$-theory).

In most of the literature and applications one restricts to the subset of topological twists which are classified by third cohomology. In the present paper we consider the degree as part of the twist. Therefore given a manifold $M$, an integer $n \in \mathbb{Z}$, and a cohomology class $z \in H^{3}(M ; \mathbb{Z})$ one has a twisted $K$-theory group $\mathrm{K}^{n+z}(M)$. A classical calculation of twisted $K$-theory is (see Example 3.14 )

$$
\mathrm{K}^{1+z}\left(S^{3}\right) \cong \mathbb{Z} / k \mathbb{Z}, \quad \mathrm{K}^{0+z}\left(S^{3}\right)=0,
$$

if $z \in H^{3}\left(S^{3} ; \mathbb{Z}\right)$ is the $k$ th multiple of the canonical generator and $k \neq 0$. Twisted $K$-theory has a product (which is trivial in the $S^{3}$-example)

$$
\cup: \mathrm{K}^{n+z}(M) \otimes \mathrm{K}^{n^{\prime}+z^{\prime}}(M) \rightarrow \mathrm{K}^{n+n^{\prime}+z+z^{\prime}}(M) .
$$

So far this picture of twisted $K$-theory has the draw-back that the group $\mathrm{K}^{n+z}(M)$ is only defined up to non-canonical isomorphism. In order to fix this group itself one must choose a cycle for the cohomology class $z$. In general, the automorphisms of cycles act nontrivially on the twisted $K$-theory groups. A detailed picture of this phenomenom has been explained in an axiomatic way in [BS05, 3.1].

The precise meaning of a cycle for a twist depends on the particular construction of twisted $K$-theory. In the literature cycles are taken as $U(1)$-gerbes, principal $P U$-bundle, bundles of Fredholm operators or maps to a model of the Eilenberg-MacLane space $K(\mathbb{Z}, 3)$. In the language of the present paper such a map would be considered as an object of the Picard- $\infty$ groupoid $K(\mathbb{Z}, 3)(M)$.

In the present paper we work with the Picard- $\infty$ groupoid of all topological twists of complex $K$-theory on $M$ which is denoted by $\operatorname{Pic}_{K}^{\text {loc }}(M)$. The specialization to third cohomology is obtained via a map of Picard- $\infty$ stacks $\underline{K(\mathbb{Z}, 3)} \rightarrow$ Pic $_{\underline{K}}^{\text {loc }}$ which is essentially unique by [AGG11].

Our way to phrase the dependence of twisted $K$-theory on the cycle, the action of the automorphism groups of cycles, and the multiplicative structure is to say that the association

$$
\mathrm{K}^{(\cdots)}(M): \quad \mathcal{H o P i c}_{\underline{K}}^{\text {loc }}(M) \rightarrow \mathrm{Ab}
$$

is a lax symmetric monoidal functor. Here $\mathcal{H o P i c} \mathrm{C}_{K}^{\text {loc }}(M)$ is the homotopy category of twists on $M$ and $\mathrm{Ab}$ denotes the category of abelian groups. After specialization to $K(\mathbb{Z}, 3)$ this realizes the axioms listed in [BS05, 3.1] and includes in addition the multiplicative structure. In the language of the present paper, the functor $\mathrm{K}^{(\cdots)}(M)$ is a graded ring, graded by the homotopy category $\mathcal{H}$ oPic $\mathrm{K}_{\mathrm{K}}^{\text {loc }}(M)$ of topological twists of $\mathrm{K}$ on $M$.

The real model for the untwisted complex $K$-theory of a manifold $M$ is the 2-periodic de Rham complex $\Omega^{*}(M)\left[b, b^{-1}\right]$, where $\operatorname{deg}(b)=-2$. It receives a multiplicative Chern character transformation

$$
\mathbf{c h}: \mathrm{K}^{*}(M) \rightarrow H^{*}\left(\Omega^{*}(M)\left[b, b^{-1}\right]\right) .
$$


which becomes an isomorphism after tensoring the domain with $\mathbb{R}$ provided $M$ is compact. If $V \rightarrow M$ is a complex vector bundle and $\nabla$ is a connection on $V$, then $\operatorname{ch}([V])$ is represented by the closed form

$$
\operatorname{Tr} \exp \left(\frac{b}{2 \pi i} R^{\nabla}\right) \in Z^{0}\left(\Omega^{*}(M)\left[b, b^{-1}\right]\right) .
$$

We now come back to the twisted case and consider an equivalence class of twists coming from a class $z \in H^{3}(M ; \mathbb{Z})$. Then one considers a closed three form $\omega \in Z^{3}\left(\Omega^{*}(M)\right)$ representing the image of the class $z$ in de Rham cohomology and forms the twisted de Rham complex $\left(\Omega^{*}(M)\left[b, b^{-1}\right], d+b \omega\right)$ by perturbing the de Rham differential with $b \omega$. The twisted de Rham complex is the correct real model of $z$-twisted $K$-theory. This is justified by the existence of a twisted version of the Chern character

$$
\mathbf{c h}: \mathrm{K}^{*+z}(M) \rightarrow H^{*}\left(\Omega^{*}(M)\left[b, b^{-1}\right], d+b \omega\right)
$$

which again becomes an isomorphism after tensoring the domain with $\mathbb{R}$ for compact $M$. This twisted Chern character has been constructed in various models [MS03], MS06a], [TX06], GT10] and is in general (with the exception of [GT10]) based on an infinitedimensional generalization of Chern-Weyl theory and the formula (1). One consequence of the present paper is a homotopy theoretic construction of the twisted Chern character.

Since the twisted $K$-group is determined by the class $z$ only up to non-canonical isomorphism it is impossible to say that the twisted Chern character is natural in the twist or compatible with products etc. Improvements in this direction have been adressed in the literature. The present paper provides a solution of the problem as a consequence of the construction of a differential twisted complex $K$-theory.

As a start we fix the commutative differential graded algebra $\mathbb{R}\left[b, b^{-1}\right]$ (with trivial differential and $\operatorname{deg}(b)=-2)$ as a real model of complex $K$-theory. In fact, there exists a map of commutative ring spectra

$$
c: \mathrm{K} \rightarrow H \mathbb{R}\left[b, b^{-1}\right]
$$

(where $H$ is the Eilenberg-MacLane equivalence between chain complexes and $H \mathbb{Z}$-module spectra) which becomes an equivalence after forming the product of $\mathrm{K}$ with the Moore spectrum $M \mathbb{R} \simeq H \mathbb{R}$. The fact that complex $K$-theory admits such a simple real model is a consequence of the property of being differentially simple (Definition 9.1). We call the triple $\widehat{\mathrm{K}}:=\left(\mathrm{K}, \mathbb{R}\left[b, b^{-1}\right], c\right)$ a differential refinement of $\mathrm{K}$.

In [BG13], Bun] the choice of such a differential refinement was the starting point for the construction of a multiplicative (untwisted) differential complex $K$-theory. In the present paper we show that this choice also suffices to construct twisted differential complex $K$-theory.

In Definition 4.10 we define, functorially in the manifold $M$, a Picard- $\infty$ groupoid $\mathcal{T}_{\mathrm{w}_{\widehat{\mathrm{K}}}}(M)$ of differential twists of $\mathrm{K}$ on $M$. A differential twist of $\mathrm{K}$ on $M$ is essentially a triple $(E, \mathcal{M}, d)$ consisting of 
- a topological K-twist $E \in \operatorname{Pic}_{\underline{K}}^{\text {loc }}(M)$,

- a sheaf of real chain complexes $\mathcal{M}$ over $M$ which is additionally a $D G$-module over the sheaf $\Omega \mathbb{R}\left[b, b^{-1}\right]$ (given by $U \mapsto \Omega^{*}(U)\left[b, b^{-1}\right]$ ),

- and an equivalence $d$ which identifies $\mathcal{M}$ with a real version $E_{\mathbb{R}}$ of $E$.

By Example 11.7 the groupoid $\mathcal{T}_{\mathrm{w}_{\widehat{\mathrm{K}}}}(M)$ in particular contains twists of the form

$$
\hat{E}:=\left(E,\left(\Omega \mathbb{R}\left[b, b^{-1}\right], d+b \omega\right), d\right),
$$

where $E$ is a topological K-twist classified by a class $z \in H^{3}(M ; \mathbb{Z})$ and $\omega \in Z^{3}\left(\Omega^{*}(M)\right)$ represents the de Rham class of $z$.

Twisted differential complex $K$-theory on $M$ is by Corollary 4.14 a lax symmetric monoidal functor (i.e. a graded ring)

$$
\widehat{\mathrm{K}}^{(\cdots)}(M): \mathcal{H o}_{\mathrm{O}} \mathcal{T}_{\widehat{\mathrm{K}}}(M) \rightarrow \mathrm{Ab}
$$

An important piece of the structure of twisted differential cohomology is the differential form contribution. More precisely the construction of twisted differential $K$-theory provides a morphism of abelian groups

$$
\mathcal{C} \text { urv }: \widehat{\mathrm{K}}^{(E, \mathcal{M}, d)}(M) \rightarrow Z^{0} \mathcal{M}(M)
$$

where $Z^{0} \mathcal{M}(M)$ are the zero-cycles in the $D G-\Omega^{*}(M)\left[b, b^{-1}\right]$-module $\mathcal{M}(M)$. The morphism $\mathcal{C}$ urv maps a differential cohomology class to its underlying 'curvature form'. This transformation can be considered as a multiplicative differential form level refinement of the Chern character (2) which is natural in the twist.

Let us make this transparent for the twist (3). In this case we get a map

$$
\mathcal{C} u r v: \widehat{\mathrm{K}}^{\hat{E}}(M) \rightarrow Z^{0}\left(\Omega^{*}(M)\left[b, b^{-1}\right], d+b \omega\right) \subset \Omega^{*}(M)\left[b, b^{-1}\right] .
$$

For $x \in \widehat{\mathrm{K}}^{\hat{E}}(M)$ the form $\mathcal{C} \operatorname{urv}(x) \in \Omega^{*}(M)\left[b, b^{-1}\right]$ satisfies the twisted cycle condition $(d+b \omega) \mathcal{C} \operatorname{urv}(x)=0$, and its cohomology class represents $\operatorname{ch}(I(x))$ in twisted de Rham cohomology, where $I(x) \in \mathrm{K}^{E}(M)$ is the underlying twisted $K$-theory class of $x$ and $\mathbf{c h}$ is the twisted Chern character map (2).

We continue this example and investigate the action of automorphisms of twists. As an application of the differential cohomology interpretation of differential twists explained in

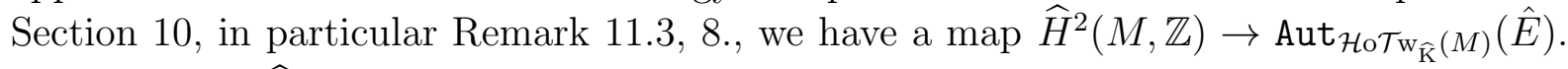
If a class $\phi \in \widehat{H}^{2}(M, \mathbb{Z})$ in second ordinary differential cohomology gives rise to such an automorphism of $\hat{E}$, then it in particular acts on the complex $\Omega^{*}\left(M, \mathbb{R}\left[b, b^{-1}\right]\right)$. In this 
case the action is given by multiplication by $\exp (-b \mathcal{C} \operatorname{urv}(\phi))$ with $\mathcal{C} \operatorname{urv}(\phi) \in \Omega^{2}(M)$. Moreover the diagram

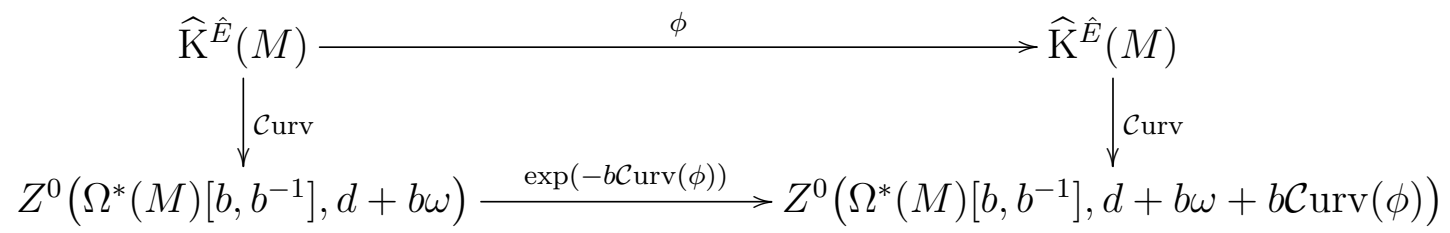

commutes. This can be interpreted as part of the statement of naturality of the twisted Chern character forms.

Given a second differential twist $\hat{E}^{\prime}$ of the form (3) we can form the product of differential twists

$$
\hat{E}+\hat{E}^{\prime}=\left(E+E^{\prime},\left(\Omega \mathbb{R}\left[b, b^{-1}\right], d+b\left(\omega+\omega^{\prime}\right)\right), d+d^{\prime}\right) .
$$

The fact that $\mathcal{C}$ urv is a symmetric monoidal transformation in particular says that the diagram

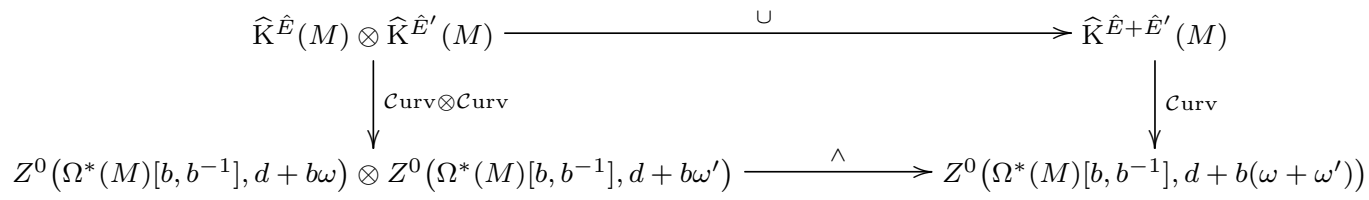

commutes. This is a differential form level statement of the fact that the twisted Chern character is compatible with products.

So far we have discussed consequences of the construction of twisted differential $K$-theory for the Chern character. A major mathematical motivation to consider differential cohomology (and its twisted versions) is the fact that it receives secondary invariants. The application (see the examples discussed in [Bun, Sec. 4]) of differential cohomology to the construction of secondary invariants is usually related with the exact sequences stated in general in Proposition 5.1. In the literature, a major part of the effort is invested in the construction of these sequences. In fact, their verification is important in order to state that the respective construction really produces a version of (twisted) differential cohomology. In our approach, similar as in [HS05], these sequences are immediate consequences of the construction.

Observe that the theory developed in the present paper does not completely provide the mathematical background for the constructions envisaged in [DFM11]. For this it would be necessary to include group actions, orbifolds and equivariant twists.

While the advantage of our homotopy theoretic construction of twisted differential $K$ theory is that it is easy to verify functorial properties and construct maps out of twisted differential $K$-theory, a shortcome is the difficulty to construct maps into differential twisted $K$-theory from differential geometry or analytic objects (see [BG13] for a more detailed discussion). 
Acknowledgements. We would like to thank David Gepner and Michael Völkl for helpful discussions. The authors are supported by the Collaborative Research Centre 1085 "Higher Invariants".

\section{Part I}

\section{Twisted cohomology via graded ring spectra}

\section{Graded ring spectra}

In this section we will, generalizing commutative (more precisely $E_{\infty}$ ) ring spectra, define the notion of a commutative graded ring spectrum. As motivation we first explain the simpler case of the generalization from commutative rings to commutative graded rings.

If $A$ is an abelian monoid, then a commutative $A$-graded ring is given by the datum of an abelian group $R_{a}$ for every $a \in A$ together with morphisms $R_{a} \otimes R_{b} \rightarrow R_{a b}$ which must satisfy associativity and commutative conditions. We let $\underline{A}$ be the discrete category on the underlying set of $A$, i.e. the objects of $\underline{A}$ are the elements of $A$ and there are only identity morphisms. The monoid structure of $A$ turns $\underline{A}$ into a symmetric monoidal category. With this in mind, a commutative $A$-graded ring can be considered as the datum of a lax symmetric monoidal functor from $\underline{A} \rightarrow \mathrm{Ab}$, where Ab denotes the symmetric monoidal category of abelian groups.

It makes sense to replace $\underline{A}$ by an arbitrary symmetric monoidal category $\mathcal{C}$ and define the category of commutative $\mathcal{C}$-graded rings to be the category of lax symmetric monoidal functors from $\mathcal{C}$ to abelian groups:

$$
\operatorname{GrRing}_{\mathcal{C}}:=\operatorname{Fun}_{\text {lax }}^{\otimes}(\mathcal{C}, \mathrm{Ab}) .
$$

Lax symmetric monoidal here means that the monoidal coherence morphisms are possibly non-invertible. The direction is such that a lax monoidal functor sends algebra objects in $\mathcal{C}$ to algebra objects in $\mathrm{Ab}$. The assignment

$$
\mathcal{S}_{y m \mathcal{M}} \rightarrow \mathcal{C}^{\text {at }}{ }^{\text {op }} \quad, \quad \mathcal{C} \mapsto \operatorname{GrRing}_{\mathcal{C}}
$$

defines a contravariant functor from the 2-category of symmetric monoidal categories and lax symmetric monoidal functors $\mathcal{S} y m \mathcal{M}$ on to the 2-category of categories.

Definition 2.1. The 2-category GrRing is defined as the Grothendieck construction of the functor (4).

Thus objects in GrRing are pairs $\left(\mathcal{C}, R_{\bullet}\right)$, where $\mathcal{C}$ is a symmetric monoidal category and $R_{\bullet}$ is a $\mathcal{C}$-graded ring. Moreover there is a functor $U:$ GrRing $\rightarrow \mathcal{S}$ ym $\mathcal{M}$ on given by $\left(\mathcal{C}, R_{\bullet}\right) \mapsto \mathcal{C}$ 
We now want to generalize the notion of graded rings to graded ring spectra. Since spectra form an $\infty$-category it is most natural to allow indexing categories which are themselves symmetric monoidal $\infty$-categories instead of ordinary symmetric monoidal categories. In the present paper we consider the $\infty$-category of symmetric monoidal $\infty$-catgeories and lax monoidal functors $\mathcal{S}_{\mathrm{ym}} \mathcal{M}_{\mathrm{n}_{\infty}}{ }^{1}$ as the home of the following web of full reflective $\infty$-subcategories

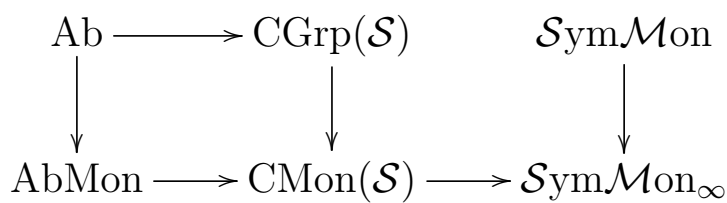

of commutative groups and monoids in the category of sets and the $\infty$-category $\mathcal{S}$ of spaces. We will not write these inclusions explicitly and hope that this abuse will not lead to confusion. Commutative groups in spaces are also called Picard- $\infty$ groupoids. Thus a Picard- $\infty$-groupoid is a symmetric monoidal $\infty$-category such that the underlying $\infty$-category is an $\infty$-groupoid with the property that every object has a tensor inverse. Note that the $\infty$-loop space functor $\Omega^{\infty}$ provides an equivalence of the $\infty$-category of connective spectra $\mathcal{S}_{\mathrm{p}}$ o with the $\infty$-category of Picard- $\infty$ groupoids.

Recall that the $\infty$-category $\mathcal{S}$ p of spectra is a symmetric monoidal $\infty$-category with respect to the smash product (see [Lur11, Section 6.3.2]).

Definition 2.2. Let $\mathcal{C}$ be a symmetric monoidal $\infty$-category. A commutative $\mathcal{C}$-graded ring spectrum is a lax symmetric monoidal functor $\mathcal{C} \rightarrow \mathcal{S}$.

In the following we illustrate this definition by relating some special cases with more classical notions.

Example 2.3. 1. Let $\mathcal{C}$ be the trivial symmetric monoidal $\infty$-category, i.e. a point considered as a group-object in spaces. Then a $\mathcal{C}$-graded ring spectrum is just a commutative ring spectrum.

2. Let $R^{\bullet}$ be an ordinary commutative $\mathcal{C}$-graded ring as in Definition 2.1, where $\mathcal{C}$ is an ordinary symmetric monoidal category. Then the collection of Eilenberg-Mac Lane spectra $H R^{\bullet}$ has the structure of a $\mathcal{C}$-graded-ring spectrum ${ }^{2}$.

3. If $\mathcal{C}$ is an ordinary abelian monoid considered as a discrete symmetric monoidal $\infty$-category, then a $\mathcal{C}$-graded ring spectrum is given by the following set of data:

(a) for every element $a \in \mathcal{C}$ a spectrum $R_{a}$,

(b) for every pair of elements $a, b \in \mathcal{C}$ a morphism $R_{a} \wedge R_{b} \rightarrow R_{a b}$,

\footnotetext{
${ }^{1}$ This is defined a the full subcategory of the $\infty$-category of $\infty$-operads spanned by symmetric monoidal $\infty$-categories, see [Lur11].

${ }^{2}$ Here we should strictly speaking write $N \mathcal{C}$ for the symmetric monoidal $\infty$-category associated to $\mathcal{C}$, but we suppress this by our general language abuse.
} 
(c) for triples $a, b, c \in \mathcal{C}$ coherence cells filling the obvious diagram.

(d) There is moreover an infinite list of higher coherence cells which is made precise by the above definition.

4. Let us consider a $\mathcal{C}$-graded ring spectrum $R$. If we apply the functor $\pi_{0}: \mathcal{S p} \rightarrow \mathrm{Ab}$, then get a lax monoidal functor $\mathcal{C} \rightarrow \mathrm{Ab}$. It has a canonical factorization over a lax monoidal functor $\mathcal{H o C} \rightarrow \mathrm{Ab}$, where $\mathcal{H o C}$ is the homotopy category of $\mathcal{C}$. Thus the homotopy of a $\mathcal{C}$-graded ring spectrum $R$ is a $\mathcal{H}$ oC-graded ring $\pi_{0}(R)$.

In the following we define an $\infty$-category of commutative graded spectra. We let $\mathcal{C}$ at ${ }_{\infty}$ denote the $\infty$-category of $\infty$-categories. We consider the functor

$$
\mathcal{S} \text { ym } \mathcal{M o n}_{\infty}^{o p} \rightarrow \mathcal{C a t}_{\infty}, \quad \mathcal{C} \mapsto \operatorname{Fun}_{\mathrm{lax}}^{\otimes}(\mathcal{C}, \mathcal{S p})
$$

For the Grothendieck construction in $\infty$-categories see [Lur09, Chapter 3].

Definition 2.4. The $\infty$-category of graded ring spectra $\operatorname{Gr} \mathcal{R} \operatorname{ing} \mathcal{S} \mathrm{p}$ is defined to be the Grothendieck construction of the functor (6).

Thus roughly speaking an object in $\operatorname{Gr} \mathcal{R} \operatorname{ing} \mathcal{S}$ p is a pair $(\mathcal{C}, R)$ of a symmetric monoidal $\infty$ category $\mathcal{C}$ and a $\mathcal{C}$-graded ring spectrum $R$. A morphism $(u, f):(\mathcal{C}, R) \rightarrow\left(\mathcal{C}^{\prime}, R^{\prime}\right)$ consists of a lax symmetric monoidal functor $f: \mathcal{C} \rightarrow \mathcal{C}^{\prime}$ and a morphisms of graded ring spectra $u: R \rightarrow f^{*} R^{\prime}$. Moreover, we have a canonical forgetful functor $\operatorname{Gr} \mathcal{R}$ ing $\mathcal{S} \mathrm{p} \rightarrow \mathcal{S}_{\mathrm{ym}} \mathcal{M}_{\infty}$.

The following lemma is an immediate consequence of the definitions.

Lemma 2.5. There is a functor

$$
\operatorname{Gr} \mathcal{R i n g} \mathcal{S} \mathrm{p} \rightarrow \operatorname{Gr} \mathcal{R i n g} \quad(\mathcal{C}, R) \mapsto\left(\mathcal{H} \circ \mathcal{C}, \pi_{0}(R)\right)
$$

We have collected some technical facts about graded ring spectra in the Appendix A.

\section{Twisted cohomology}

A classical instance of twisted cohomology is the cohomology of a space with coefficients in a locally constant sheaf, for example the orientation sheaf. Twisted $K$-theory has been introduced in [DK70]. Much later, motivated by applications in physics it became an intensively studied object, see Section 1.2 of the introduction. General approaches to twisted cohomology are given in [MS06b] and [ABG10, ABG11. This last reference can be considered as a starting point for the approach taken in the present paper.

In this section we review twisted cohomology and phrase it in the framework of graded rings and graded ring spectra. The construction serves as a model for the slightly more involved case of twisted differential cohomology.

We first need some sheaf-theoretic notation. In the present paper we consider sheaves with values in a presentable $\infty$-category $\mathcal{C}$. An ordinary category is considered as an 
$\infty$-category via its nerve. By $\mathcal{M f}$ we denote the site of smooth manifolds with the pretopology of open coverings. By $\mathrm{Sh}_{\mathcal{C}}$ we denote the $\infty$-category of sheaves on the site $\mathcal{M f}$ with target $\mathcal{C}$. We use the symbol $\operatorname{Sh}_{\mathcal{C}}(M)$ in order to denote the category of sheaves on the restricted site $\mathcal{M f} / M$. For a sheaf $A \in \operatorname{Sh}_{\mathcal{C}}$ we let $A_{\mid M} \in \operatorname{Sh}_{\mathcal{C}}(M)$ denote the restriction of $A$ to $\mathcal{M f} / M$. For presheaves we will use similar conventions and the symbol PSh. A reference for sheaves with values in $\infty$-categories is [Lur09]. See also the note BNV13] for some sheaf theory in this setting.

The diagram of reflective subcategories (5) induces a corresponding diagram of reflective subcategories of sheaves on $\mathcal{M f}$ or any other site with values in the respective $\infty$ categories. We will use these inclusions without further notice. Sheaves of groupoids will also be referred to as stacks. In particular, a sheaf of Picard- $\infty$ groupoids is called a Picard- $\infty$ stack.

An object $X \in \mathcal{C}$ gives rise to a constant presheaf $C_{X} \in \operatorname{PSh}_{\mathcal{C}}$ defined by $C_{X}(N):=X$ for all objects $N \in \mathcal{M f}$. By $\hat{C}_{X} \in \mathrm{Sh}_{\mathcal{C}}$ we denote its sheafification.

Definition 3.1. Let $M$ be a smooth manifold. A sheaf $\mathcal{F} \in \operatorname{Sh}_{\mathcal{C}}(M)$ is called constant if there exists an object $X \in \mathcal{C}$ and an equivalence $\left(\hat{C}_{X}\right)_{\mid M} \cong \mathcal{F}$.

A sheaf $\mathcal{F} \in \operatorname{Sh}_{\mathcal{C}}(M)$ is called locally constant if every point $m \in M$ has a neighbourhood $U \subseteq M$ such that $\mathcal{F}_{\mid U} \in \mathrm{Sh}_{\mathcal{C}}(U)$ is constant. We write

$$
\operatorname{Sh}_{\mathcal{C}}^{\text {loc }}(M) \subseteq \operatorname{Sh}_{\mathcal{C}}(M)
$$

for the full subcategory of locally constant sheaves.

Definition 3.2. For an object $X \in \mathcal{C}$ of a presentable $\infty$-category $\mathcal{C}$ we define the sheaf $\underline{X} \in \mathrm{Sh}_{\mathcal{C}}$ such that its evaluation on the manifold $M$ is given by $\underline{X}(M):=X^{M^{\text {top }}}$.

In this definition, $M^{\text {top }}$ is the underlying space of $M$, and we use that a presentable $\infty$ category is cotensored over spaces. We refer to the appendix of [BG13] for the verification of the the following facts.

Proposition 3.3. $\quad$ 1. The presheaf $M \mapsto X^{M^{\text {top }}}$ is indeed a sheaf.

2. The sheaf $\underline{X}$ is constant. In fact, we have an equivalence $\underline{X} \cong \hat{C}_{X}$.

If $\mathcal{C}$ is a (closed) symmetric monoidal $\infty$-category, then the categories $\operatorname{PSh}_{\mathcal{C}}(M), \operatorname{Sh}_{\mathcal{C}}(M)$ and $\mathrm{Sh}_{\mathcal{C}}^{\text {loc }}(M)$ also carry (closed) symmetric monoidal structures given by the objectwise tensor product. Thus it makes sense to speak of (commutative) algebra and module objects in these categories. It turns out that algebras in (locally constant) sheaves are the same as (locally constant) sheaves of algebras.

Proposition 3.4. Let $\mathcal{C}$ be a (closed) presentable symmetric monoidal $\infty$-category. Then we have equivalences

$$
\mathrm{CAlg}\left(\operatorname{Sh}_{\mathcal{C}}(M)\right) \simeq \operatorname{Sh}_{\mathrm{CAlg}(\mathcal{C})} \quad \text { and } \quad \operatorname{CAlg}\left(\operatorname{Sh}_{\mathcal{C}}^{\operatorname{loc}}(M)\right) \simeq \operatorname{Sh}_{\mathrm{CAlg}(\mathcal{C})}^{\operatorname{loc}}(M) .
$$

If $X \in \mathcal{C}$ is a commutative algebra object, the sheaf $\underline{X}$ is a locally constant sheaf of commutative algebras and we have similar equivalences

$$
\operatorname{Mod}_{\underline{X}}(M) \simeq \operatorname{Sh}_{\operatorname{Mod}_{X}}(M) \quad \text { and } \quad \operatorname{Mod}_{\underline{X}}^{\operatorname{loc}}(M) \simeq \operatorname{Sh}_{M^{\text {loc }} X}^{\text {loc }}(M) .
$$


Proof. We give the proof for the first equivalences. The argument for the second assertion is similar. For categories of presheaves we have an evident equivalence $\operatorname{CAlg}\left(\operatorname{PSh}_{\mathcal{C}}(M)\right) \simeq$ $\mathrm{PSh}_{\mathrm{CAlg}(\mathcal{C})}$ which directly follows from the definition resp. universal property of the point wise tensor product on functor categories [Lur11, Section 2.2.5.]. Thus it is enough to check that under this equivalence sheaves and locally constant sheaves are preserved. For the case of sheaves this follows since the sheaf condition is a limit condition and the forgetful functor which forgets algebra structures commutes with limits in any closed symmetric monoidal $\infty$-category [Lur11, Section 3.2.2.]. For the second equivalence we use that the first obviously commutes with restriction. It therefore suffices to show the equivalence for constant sheaves. Here we use again that the forgetful functor that forgets the algebra structure commutes with limits, so in particular with the tensoring used in 3.3 in order to define constant sheaves.

For a commutative ring spectrum $R$ the $\infty$-category of module spectra over $R$ will be denoted $\operatorname{Mod}_{R}$. By Pic Pic $_{R}$ we denote the maximal Picard- $\infty$-groupoid inside of $\operatorname{Mod}_{R}$, i.e. the objects are $R$-module spectra which admit a tensor inverse, and the morphisms are the invertible $R$-module maps. Similarly by $\operatorname{Pic}_{\underline{R}}^{\text {loc }}(M)$ we denote the maximal Picard- $\infty$ groupoid inside the $\infty$-category $\operatorname{Sh}_{\operatorname{Mod}_{R}}^{\text {loc }}(M)$ of locally constant sheaves of $R$-modules on $M$.

Definition 3.5. The objects of $\operatorname{Pic}_{\underline{R}}^{\text {loc }}(M)$ are called $R$-twists on $M$.

Let us list a few properties of $R$-twists that we will need later. The proofs are given in Appendix B.

Proposition 3.6. $\quad$ 1. The assignment $M \mapsto \mathrm{Pic}_{R}^{\mathrm{loc}}(M)$ itself forms a Picard- $\infty$-stack on $\mathcal{M f}$, i.e.

$$
\operatorname{Pic}_{\underline{R}}^{\text {loc }} \in \operatorname{Sh}_{\mathrm{CGrp}(\mathcal{S})} \text {. }
$$

2. For every manifold $M$ there is a canonical equivalence of Picard- $\infty$-groupoids

$$
\operatorname{Pic}_{\underline{R}}^{\mathrm{loc}}(M) \cong\left(\mathrm{Pic}_{R}\right)^{M^{\text {top }}}=\underline{\mathrm{Pic}_{R}}(M) \text {. }
$$

3. Every $R$-twist $E \in \operatorname{Pic}_{R}^{\text {loc }}(M)$ on $M$ is homotopy invariant. That means that for every map $N \rightarrow N^{\prime}$ in $\overline{\mathcal{M}} \mathrm{f} / M$ which is a homotopy equivalence (not necessarily over $M)$ the induced map $E\left(N^{\prime} \rightarrow M\right) \rightarrow E(N \rightarrow M)$ is an equivalence of $R$-modules.

For any manifold $M$ the evaluation provides a lax symmetric monoidal transformation

$$
\Gamma: \operatorname{Pic}_{\underline{R}}^{\text {loc }}(M) \rightarrow \mathcal{S p} \quad E \mapsto \Gamma(M, E):=E(M) .
$$

Hence the pair $\left(\operatorname{Pic}_{\underline{R}}^{\text {loc }}(M), \Gamma\right)$ is a graded ring spectrum which we denote $\mathcal{R}(M)$.

Proposition 3.7. Let $R \in \mathrm{CAlg}(\mathcal{S} \mathrm{p})$ be a commutative ring spectrum. Then the assignment $M \mapsto \mathcal{R}(M)$ canonically refines to a sheaf of graded ring spectra $\mathcal{R} \in \mathrm{Sh}_{\mathrm{Gr} \mathcal{R} \text { ing } \mathcal{S} \mathrm{p}}$. 
Proof. By Proposition A.9 we have an object

$$
F \in \operatorname{Sh}_{\text {GrRing } \mathcal{S p}}, \quad \mathcal{M f} \ni M \mapsto F(M):=\left(\operatorname{Sh}_{\mathcal{S p}}(M), \Gamma(M, \ldots)\right) .
$$

We now want to change the grading component of $F$ to Pic loc . Therefore we apply the change of grading Proposition A.5 to $F$, the sheaf $G:=\operatorname{Pic}_{\underline{R}}^{\text {loc }} \in \operatorname{Sh}_{\mathcal{S y m}_{\mathbf{M} n_{\infty}} \text {, and the }}$ transformation $\eta: \mathrm{Pic}_{R}^{\text {loc }} \rightarrow \mathrm{Sh}_{\mathcal{S} \mathrm{p}}$ which forgets invertibility of objects and morphisms and the $R$-module structure in order to get a new sheaf of graded ring spectra $\mathcal{R} \in \mathrm{Sh}_{\mathcal{S}}$.

The construction of the graded ring spectrum $\mathcal{R}$ from $R$ is even functorial in $R$. More precisely we have:

Proposition 3.8. The construction $R \mapsto \mathcal{R}$ naturally refines to a functor

$$
\mathrm{CAlg}(\mathcal{S p}) \rightarrow \operatorname{Sh}_{\mathrm{Gr} \mathcal{R} i n g} \mathcal{S}_{\mathrm{p}}
$$

Proof. We describe the adjoint functor $\operatorname{CAlg}(\mathcal{S} \mathrm{p}) \times \mathcal{M} \mathrm{f}^{o p} \rightarrow \operatorname{Gr} \mathcal{R}$ ing $\mathcal{S}$ p. We apply the change of grading statement (Proposition A.5) together with the sheaf-refinement given in Proposition A.9 to the $\infty$-category $\mathcal{D}:=\operatorname{CAlg}(\mathcal{S p}) \times \mathcal{M} \mathrm{f}^{o p}$, the functors

$$
F: \operatorname{CAlg}(\mathcal{S p}) \times \mathcal{M} \mathrm{f}^{o p} \stackrel{\mathrm{pr}_{2}}{\longrightarrow} \mathcal{M} \mathrm{f}^{o p} \stackrel{8}{\longrightarrow} \operatorname{Gr} \mathcal{R i n g} \mathcal{S} \mathrm{p}
$$

and

$$
G: \operatorname{CAlg}(\mathcal{S p}) \times \mathcal{M} \mathrm{f}^{o p} \rightarrow \mathcal{S y m}_{\mathcal{M}} \operatorname{Mon}_{\infty}, \quad(R, M) \mapsto \operatorname{Pic}_{\underline{R}}^{h}(M),
$$

and the lax symmetric monoidal transformation

$$
\eta: G \rightarrow \operatorname{Sh}_{\mathcal{S} \mathrm{p}}, \quad \operatorname{Pic}_{\underline{R}}^{h}(M) \rightarrow \operatorname{Sh}_{\mathcal{S} \mathrm{p}}(M)
$$

We can now define twisted cohomology. In order to simplify the notation we write $E(M)$ for the evaluation of a sheaf $E$ on $\mathcal{M f} / M$ on $M \stackrel{\text { id }}{\rightarrow} M$.

Definition 3.9. Given a manifold $M$ with $R$-twist $E \in \mathcal{H}_{\mathrm{oPic}} \mathrm{loc}_{\underline{R}}^{\mathrm{l}}(M)$ we define the $E$ twisted $R$-cohomology of $M$ by

$$
R^{E}(M):=\pi_{0}(E(M))
$$

Remark 3.10. The reader might wonder why there is no degree in the twisted cohomology groups $R^{E}(M)$. He might also know that cohomology groups are usually twisted by classes in $\left[M, \mathrm{BGL}_{1}(R)\right]$ where $\mathrm{GL}_{1}(R)$ is the space of units of $R$. The reason is that $\mathrm{Pic}_{\underline{R}}^{\text {loc }}(M)$ encodes both, the degree and the twist.

More precisely homotopy classes of maps $\left[M, \mathrm{BGL}_{1}(R)\right]$ classify those $R$-twists $E \in$ $\mathcal{H o P i c}_{\underline{R}}^{\text {loc }}(M)$ which are trivial when restricted to points. For such a twists $E$ and integer $n \in \mathbb{Z}$ the twisted cohomology of degree $n$ can be expressed in the present language 
in the form $R^{E[n]}(M)$, where $E[n] \in \mathcal{H}_{\mathrm{oPic}} \mathrm{C}_{\underline{R}}^{\text {loc }}(M)$ is the shift of $n$.

In general, $\operatorname{Pic}_{\underline{R}}^{\text {loc }}(M)$ contains more then just locally trivial twists (do not confuse with locally constant) and shifts. For example the $E(1)$-local sphere contains exotic elements in its Picard group [HMS94]. Another example is given by the Eilenberg-MacLane spectrum $H R$ for $R$ a Dedekind ring with nontrivial class group, such as $\mathbb{Z}[\sqrt{-5}]$.

Corollary 3.11. For each manifold $M$ the twisted cohomology $R^{*}(M)$ forms a graded ring which is graded over the homotopy category $\mathcal{H o P i c}_{R}^{\text {loc }}(M)$. Moreover the assignment

$$
M \mapsto\left(\mathcal{H o P i c}{ }_{R}^{\text {loc }}(M), R^{*}(M)\right)
$$

forms a functor $\mathcal{M} \mathrm{f}^{o p} \rightarrow$ GrRing.

Corollary 3.11 says more concretely that we have the following structures:

1. For every $R$-twist $E \in \mathcal{H}_{\mathrm{oPic}} \mathrm{C}_{\underline{R}}^{\text {loc }}(M)$ on $M$ we have an abelian group $R^{E}(M)$.

2. For every pair $E, E^{\prime}$ of $R$-twists on $M$ we have a multiplication morphism

$$
R^{E}(M) \otimes R^{E^{\prime}}(M) \rightarrow R^{E+E^{\prime}}(M)
$$

where $E+E^{\prime}$ denotes the symmetric monoidal pairing in $\mathcal{H o P i c}_{R}^{\text {loc }}(M)$. The multiplication is associative, commutative, and has a unit in the obious sense.

3. For every morphisms $E \rightarrow E^{\prime}$ between $R$-twists on $M$ we have an isomorphism

$$
R^{E}(M) \stackrel{\sim}{\rightarrow} R^{E^{\prime}}(M)
$$

of abelian groups. This assignment is functorial and compatible with the product in the obvious sense.

4. For every smooth map $f: M \rightarrow N$ and $R$-twist $E$ on $M$ we have a morphism

$$
R^{E}(N) \rightarrow R^{f^{*} E}(M)
$$

which is compatible with all the other structures.

Further features of twisted cohomology are:

5. For every map of commutative ring spectra $u: R \rightarrow R^{\prime}$ and $R$-twist $E \in \mathcal{H}_{\text {oPic }}^{\text {loc }}(M)$ we get an $R$-twist $u_{*} E \in \mathcal{H} \mathrm{oPic}_{\underline{R^{\prime}}}^{\text {loc }}(M)$ and a map $u_{*}: R^{E}(M) \rightarrow R^{\prime, u_{*} E}(M)$.

6. If $E$ is an $R$-twist on $M$ and $U, V \subseteq M$ are open subsets such that $M=U \cup V$, then we have a long exact Mayer-Vietoris sequence

$$
\cdots \rightarrow R^{E}(M) \rightarrow R^{E_{\mid U}}(U) \oplus R^{E_{\mid V}}(V) \rightarrow R^{E_{\mid U \cap V}}(U \cap V) \rightarrow R^{E[1]}(M) \rightarrow \ldots
$$

which is compatible with the various other structures. This follows from the fact that $\mathcal{R}$ is a sheaf of graded ring spectra. 
The idea to calculate $R^{E}(M)$ using a Mayer-Vietoris sequence (9) is to cover the manifold $M$ by open subspaces on which the twist can be trivialized. The allows to express the twisted cohomology of these subspaces in terms of untwisted cohomology. It remains to determine the maps in this sequence.

Example 3.12. In the following we consider this problem when $M$ is a sphere $S^{k}$ for some $k \in \mathbb{N}$. In this case we can determine the maps explicitly in terms of an invariant of the twist defined in Definition 3.13. This invariant will also play an important role in our general theory later, e.g. in the proof of Lemma 8.10.

We identify $S^{k} \subset S^{k+1}$ with the equator and choose a base point $p \in S^{k}$. We further let $D_{ \pm} \subset S^{k+1}$ be the complements of the north- and south poles.

Assume that $E \in \operatorname{Pic}_{R}^{\text {loc }}\left(S^{k+1}\right)$ is a twist which locally is a shift of $R$. Then we can choose an identification $E_{\mid p} \cong R[n]$ of $R$-modules for some $n \in \mathbb{Z}$.

Since $E$ is homotopy invariant by Proposition 3.6 we can extend this identification to trivializations

$$
\phi_{ \pm}: E_{\mid D_{ \pm}} \stackrel{\sim}{\rightarrow} \underline{R[n]}{ }_{D_{ \pm}} .
$$

Definition 3.13. For $k \geq 0$ we define the class

$$
\nu(E) \in\left\{\begin{array}{cc}
\pi_{k}(R) & k \geq 1 \\
\pi_{0}(R)^{\times} & k=0
\end{array}\right.
$$

such that $1 \oplus \nu(E) \in R^{0}\left(S^{k}\right) \cong \pi_{0}(R) \oplus \pi_{k}(\mathbb{R})$ is the image of $1 \in R^{0}\left(D_{+}\right)$under the map

$$
R^{0}\left(D_{+}\right) \stackrel{\phi_{+}^{-1}}{\rightarrow} \pi_{0}\left(E[-n]_{\mid D_{+}}\left(D_{+}\right)\right) \stackrel{\text { restr. }}{\rightarrow} \pi_{0}\left(E[-n]_{\mid S^{k}}\left(S^{k}\right)\right) \cong \pi_{0}\left(E[-n]_{\mid D^{-}}\left(S^{k}\right)\right) \stackrel{\phi_{-}}{\rightarrow} R^{0}\left(S^{k}\right) .
$$

One easily checks that $\nu(E)$ does not depend on the choices. In fact, the trivializations $\phi_{ \pm}$are uniquely determined up to equivalence by the choice of the trivialization at the point $p$. In the construction of $\nu(E)$ we compose $\phi_{+}^{-1}$ with $\phi_{-}$so that this choice drops out.

The twisted cohomology $R^{E}\left(S^{k+1}\right)$ can be calculated using the Mayer-Vietoris sequence (9). Using the class $\nu(E)$ this sequence can be identified with the explicit sequence

$$
\cdots \rightarrow \underset{\pi_{k+1-n}(R)}{\pi_{1-n}(R)} \rightarrow R^{E}\left(S^{k}\right) \rightarrow \underset{\pi_{-n}(R)}{\pi_{-n}(R)} \stackrel{\left(\begin{array}{c}
x \\
y
\end{array}\right) \mapsto\left(\begin{array}{c}
x-y \\
\nu(E) x
\end{array}\right)}{\longrightarrow} \underset{\substack{\pi_{-n}(R) \\
\pi_{k-n}(R)}}{\oplus} \rightarrow \ldots
$$

Example 3.14. The reader may have seen the example of twisted complex $K$-theory. Let $\mathrm{K}$ denote the complex $K$-theory spectrum. Locally trivial unshifted K-twists $E$ of $S^{3}$ are classified up to equivalence by the invariant $\nu(E) \in \pi_{2}(\mathrm{~K}) \cong \pi_{3}\left(\mathrm{BGL}_{1}(\mathrm{~K})\right)$. Let us 
identify $\pi_{2 i}(\mathrm{~K}) \cong \mathbb{Z}$ for all $i \in \mathbb{Z}$ using powers of the Bott element. If $\nu(E)$ corresponds to the integer $n \in \mathbb{Z}$, then $\mathrm{K}^{E[1]}\left(S^{3}\right)$ fits into the sequence

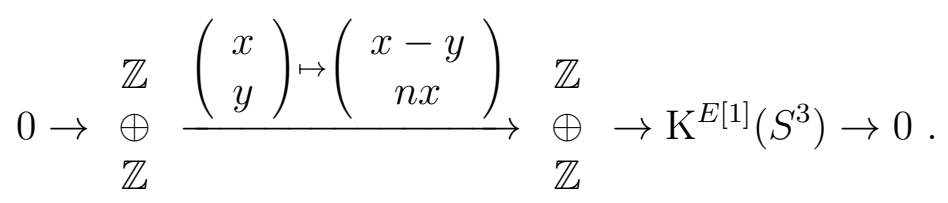

This implies the usual calculation: $\mathrm{K}^{E[1]}\left(S^{3}\right) \cong \mathbb{Z} / n \mathbb{Z}$, and by a similar argument, $\mathrm{K}^{E}\left(S^{3}\right)=0$.

\section{Twisted differential cohomology}

In this section we introduce twisted differential cohomology. The idea of differential cohomology is to combine cohomology classes on manifolds with corresponding differential form data in a homotopy theoretic way. Using differential cohomology one can encode local geometric information. For example, while the K-theory class of a vector bundle only encodes homotopy theoretic data, the differential $K$-theory class of a vector bundle with connection contains the information on Chern character forms, see [HS05], [BS09], SS10] for a detailed account. Differential extensions of arbitrary generalized cohomology theories were first defined in [HS05. We refer to [BG13] and Bun for the approach to differential cohomology on which the present paper is based. The main goal of the present section is to develop a general theory of twisted and multiplicative differential cohomology based on the notion of graded ring spectra.

A commutative ring spectrum $R$ represents a multiplicative cohomology theory. As explained in [BG13], [Bun], in order to define the multiplicative differential $R$-cohomology on manifolds we have to choose a differential refinement of $R$. In Section 3 we have seen that $R$ gives rise to a twisted cohomology theory which is naturally encoded in the sheaf of graded ring spectra $\mathcal{R}$. We shall see in the present section that the choice of a differential refinement of $R$ naturally determines a sheaf of graded ring spectra (Proposition 4.12 which encodes the twisted differential $R$-cohomology. In order to be able to study transformations between different differential cohomology theories we introduce the $\infty$-category of differential ring spectra.

Let $\mathrm{Ch}$ denote the ordinary symmetric monoidal category of chain complexes. If we formally invert the class of quasi-isomorphisms in $\mathrm{Ch}$, then we obtain an $\infty$-category $\mathrm{Ch}_{\infty}$. The natural map $\iota: \mathrm{Ch} \rightarrow \mathrm{Ch}_{\infty}$ is a lax symmetric monoidal functor. Furthermore, the Eilenberg-MacLane equivalence gives an equivalences (see [Lur11, Theorem 8.1.2.13] for details) of symmetric monoidal $\infty$-categories

$$
H: \mathrm{Ch}_{\infty} \stackrel{\sim}{\rightarrow} \operatorname{Mod}_{H \mathbb{Z}},
$$

where $\operatorname{Mod}_{H \mathbb{Z}}$ denotes module spectra over the Eilenberg-Mac Lane spectrum $H \mathbb{Z}$. By abuse of notation, for $C \in \mathrm{Ch}$ we write $H C:=H \iota(C)$. Note that the category of commutative algebras $\mathrm{CAlg}(\mathrm{Ch})$ in $\mathrm{Ch}$ is the category of commutative differential graded 
algebras (CDGAs). Hence for a CDGA $A$ we get a commutative algebra spectrum $H A \in \mathrm{CAlg}\left(\operatorname{Mod}_{H \mathbb{Z}}\right)$. We consider $\mathbb{R}$ as a CDGA concentrated in degree 0 and get the commutative algebra $H \mathbb{R}$. We write $\mathrm{Ch}_{\mathbb{R}}:=\operatorname{Mod}_{\mathbb{R}}$ for the category of chain complexes of real vector spaces. Note that $\mathrm{CAlg}\left(\mathrm{Ch}_{\mathbb{R}}\right)$ is the category of real commutative differential graded algebras (CDGAs). The composition of the localization and the Eilenberg-Mac Lane equivalence restricts to a functor $\mathrm{CAlg}\left(\mathrm{Ch}_{\mathbb{R}}\right) \rightarrow \mathrm{CAlg}\left(\operatorname{Mod}_{H \mathbb{R}}\right)$.

Definition 4.1. A differential refinement of a commutative ring spectrum $R$ is a triple $(R, A, c)$ consisting of a $C D G A A$ over $\mathbb{R}$ together with an equivalence

$$
c: R \wedge H \mathbb{R} \stackrel{\sim}{\rightarrow} H A
$$

in $\mathrm{CAlg}\left(\operatorname{Mod}_{H \mathbb{R}}\right)$.

It has been shown in [Shi07] that one can model every $H \mathbb{R}$-algebra by a CDGA. In our language this means that the functor

$$
\mathrm{CAlg}\left(\mathrm{Ch}_{\mathbb{R}}\right) \rightarrow \mathrm{CAlg}\left(\operatorname{Mod}_{H \mathbb{R}}\right), \quad A \mapsto H A
$$

is essentially surjective. In particular, every commutative ring spectrum admits a differential refinement.

Definition 4.2. We define the $\infty$-category of commutative differential ring spectra as the pullback

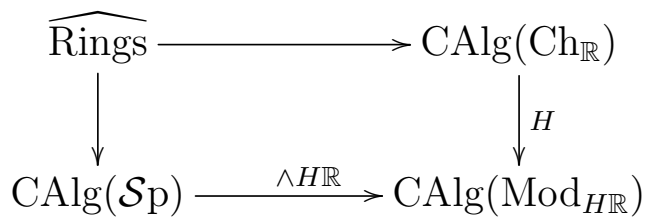

By construction, the objects of $\widehat{\text { Rings }}$ are differential refinements of commutative ring spectra.

Remark 4.3. In many relevant cases there exist a differential refinement of $R$ whose underlying CDGA is the graded ring $\pi_{*}(R) \otimes \mathbb{R}$ with trivial differentials. In this case $R \wedge H \mathbb{R}$ is called formal. For example, if $\pi_{*}(R) \otimes \mathbb{R}$ is free as a commutative $\mathbb{R}$-algebra or arises from a free algebra by inverting elements, then $R \wedge H \mathbb{R}$ is formal. In this case there is an equivalence $c$ which is uniquely determined up to homotopy by the property that it induces the canonical identification on homotopy groups [Bun, Sec. 4.6].

Example 4.4. 1. For the Eilenberg-MacLane spectrum $H \mathbb{Z}$ of $\mathbb{Z}$ we choose a real model whose underlying CDGA is $\mathbb{R}$ concentrated in degree 0 .

2. For the complex $K$-theory spectrum $K$ we have a real model with underlying CDGA given by $\mathbb{R}\left[b, b^{-1}\right]$ with trivial differentials, where $\operatorname{deg}(b)=-2$. The isomorphism $c$ is the Chern character. 
3. For the connective spectrum tmf of topological modular forms we can choose a real model with underlying CDGA given by $\mathbb{R}\left[c_{4}, c_{6}\right]$ with trivial differentials, where $\operatorname{deg}\left(c_{4}\right)=8$ and $\operatorname{deg}\left(c_{6}\right)=12$. Again, the map $c$ is unique up to equivalence.

Note that there exist non-formal examples.

The sheaf of smooth real differential forms with the de Rham differential is a sheaf of CDGA's on the site of smooth manifolds, i.e. an object $\Omega^{*} \in \mathrm{Sh}_{\mathrm{CAlg}\left(\mathrm{Ch}_{\mathbb{R}}\right)}$ which associates to every smooth manifold $M$ the smooth real de Rham complex $\left(\Omega^{*}(M), d\right)$. For a CDGA $A$ over $\mathbb{R}$ we define the sheaf of CDGA's of differential forms with values in $A$ by

$$
\Omega A:=\Omega \otimes_{\mathbb{R}} \underline{A} \in \operatorname{Sh}_{\mathrm{CAlg}\left(\mathrm{Ch}_{\mathbb{R}}\right)} .
$$

where $A$ is the constant sheaf of CDGA's associated to $A$ (see Definition 3.2 and Proposition 3.3

In general, a presheaf with values in the 1-category of chain complexes $\mathcal{M} \in \mathrm{PSh}_{\mathrm{Ch}}$ is a sheaf precisely if for every $n \in \mathbb{Z}$ its degree- $n$ component $\mathcal{M}_{n}$ is a sheaf of abelian groups. This property can easily be checked in the case of $\Omega$ or $\Omega A$.

We now extend the localization $\iota: \mathrm{Ch} \rightarrow \mathrm{Ch}_{\infty}$ to presheaves by post-composition. Let us consider a sheaf $\mathcal{M} \in \mathrm{Sh}_{\mathrm{Ch}}$. Then in general $\iota(\mathcal{M})$ is only a presheaf with values in $\mathrm{Ch}_{\infty}$. But if $\mathcal{M}$ is a complex of modules over the sheaf of rings of smooth real-valued functions on $\mathcal{M f}$, then it is a sheaf. The argument employes the existence of smooth partitions of unity. In particular, $\iota(\Omega)$ and $\iota(\Omega A)$ are sheaves. More generally, the objectwise localization $\operatorname{Mod}_{\Omega A} \rightarrow \operatorname{Mod}_{\iota(\Omega A)}$ preserves sheaves.

Example 4.5. On the other hand, consider for example the sheaf of complexes $\mathbb{R}$ concentrated degree zero given by locally constant $\mathbb{R}$-valued functions. Then $\iota(\underline{\mathbb{R}})$ is not a sheaf but $\iota(\underline{\mathbb{R}}) \rightarrow \iota(\Omega)$ is the sheafification.

We now fix a smooth manifold $M$. A morphism between objects in $\operatorname{Mod}_{\Omega A}(M)$ becomes an equivalence under the localization $\iota: \operatorname{Mod}_{\Omega A}(M) \rightarrow \operatorname{Mod}_{\iota(\Omega A)}(M)$ if and only if it is a quasi-isomorphism between complexes of sheaves.

Definition 4.6. 1. A sheaf $\mathcal{M} \in \operatorname{Mod}_{\Omega A}(M)$ is called $K$-flat if the functor

$$
\mathcal{M} \otimes_{\left.\Omega A\right|_{M}} \cdots: \operatorname{Mod}_{\Omega A}(M) \rightarrow \operatorname{Mod}_{\Omega A}(M)
$$

preserves quasi-isomorphisms [Hin97].

2. A sheaf $\mathcal{M} \in \operatorname{Mod}_{\Omega A}(M)$ is called invertible, if there is an object $\mathcal{N} \in \operatorname{Mod}_{\Omega A}(M)$ such that $\mathcal{M} \otimes_{\left.\Omega A\right|_{M}} \mathcal{N}$ is isomorphic to $\Omega A$.

3. A sheaf $\mathcal{M} \in \operatorname{Mod}_{\Omega A}(M)$ is called weakly locally constant if $\iota(\mathcal{M})$ is locally constant.

4. By $\operatorname{Pic}_{\Omega A}^{\text {wloc,fl }} \in \operatorname{Sh}_{\mathrm{CGrp}(\mathcal{S})}$ we denote the Picard- $\infty$-stack (actually a Picard-1-stack) which associates to every manifold $M$ the Picard-1-groupoid $\mathrm{Pic}_{\Omega A}^{\text {wloc,fl }}(M)$ of invertible, $K$-flat and weakly locally constant objects in $\operatorname{Mod}_{\Omega A}(M)$. 
Example 4.7. Note that $\Omega A$ is weakly locally constant. In fact, it is weakly constant since we have an equivalence $\iota(A) \stackrel{\sim}{\rightarrow} \iota(\Omega A)$ The groupoid Pic $\mathrm{c}_{\Omega A}^{\text {wloc,fl}}(M)$ is therefore welldefined and contains all modules that are shifts of $\Omega A_{\mid M}$, i.e. modules of the form $\Omega A[n]_{\mid M}$ for $n \in \mathbb{Z}$. But $\Omega A[n]_{\mid M}$ and $\Omega A[m]_{\mid M}$ can be isomorphic if $A$ is periodic, i.e. contains a unit in degree $m-n$.

Remark 4.8. Because of the additional conditions of $K$-flatness and weakly locally constantness in general the inclusion $\mathrm{Pic}_{\Omega A}^{\text {wh,f }} \subseteq \mathrm{Pic}_{\Omega A}$ is proper as the following example shows.

We shall give an example of a weakly locally constant non $K$-flat object in Pic $\mathrm{C}_{\Omega}$. Let $L_{\lambda} \rightarrow S^{1}$ be the flat one-dimensional real vector bundle with connection $\nabla^{\lambda}$ with holonomy $\lambda \in \mathbb{R}^{>0}$ and $\Omega\left(S^{1}, L_{\lambda}\right)$ be the complex of smooth forms with coefficients in $L$. It is a module over the de Rham complex $\Omega\left(S^{1}\right) \cong \Omega\left(S^{1}, L_{1}\right)$, and we have the rule

$$
\Omega\left(S^{1}, L_{\lambda}\right) \otimes_{\Omega\left(S^{1}\right)} \Omega\left(S^{1}, L_{\lambda^{\prime}}\right) \cong \Omega\left(S^{1}, L_{\lambda \lambda^{\prime}}\right) .
$$

In particular, $\Omega\left(S^{1}, L_{\lambda}\right) \in$ Pic $_{\Omega\left(S^{1}\right)}$, and its tensor inverse is given by $\Omega\left(S^{1}, L_{\lambda^{-1}}\right)$. The complex $\Omega\left(S^{1}, L_{\lambda}\right)$ can be identified with

$$
C^{\infty}\left(S^{1}\right) \stackrel{f \mapsto f^{\prime}-\log (\lambda) f}{\longrightarrow} C^{\infty}\left(S^{1}\right)
$$

We have

$$
H^{*}\left(\Omega\left(S^{1}, L_{\lambda}\right)\right) \cong\left\{\begin{array}{cc}
\mathbb{R} \oplus \mathbb{R}[1] & \lambda=1 \\
0 & \lambda \neq 1
\end{array}\right.
$$

If $\lambda \in \mathbb{R}^{>0} \backslash\{1\}$, then the morphism $0 \rightarrow \Omega\left(S^{1}, L_{\lambda}\right)$ is a quasi-isomorphism. Its tensor product with $\Omega\left(S^{1}, L_{\lambda^{-1}}\right)$ is $0 \rightarrow \Omega\left(S^{1}\right)$ which is not a quasi-isomorphism. It follows that $\Omega\left(S^{1}, L_{\lambda^{-1}}\right)$ is not $K$-flat. Thus if we set $A:=\Omega\left(S^{1}\right)$, then $\mathcal{M}:=\Omega \otimes_{\mathbb{R}} \Omega\left(S^{1}, L_{2}\right) \in$ Pic $_{\Omega A}$ is weakly locally constant but not $K$-flat.

We observe that for every manifold $M$ the transformation

$$
\operatorname{Pic}_{\Omega A}^{\mathrm{wloc}, \mathrm{fl}}(M) \rightarrow \operatorname{Mod}_{\iota(\Omega A)}^{\mathrm{loc}}(M),
$$

is symmetric monoidal (and not just lax symmetric monoidal). More concretely, the condition of $K$-flatness ensures that the tensor product of sheaves of complexes is equivalent to the derived tensor product. Hence it preserves invertible objects. If we further compose (10) with the symmetric monoidal Eilenberg Mac-Lane equivalence $\operatorname{Mod}_{\iota(\Omega A)}^{\text {loc }} \stackrel{\sim}{\rightarrow} \operatorname{Mod}_{H \Omega A}^{\text {loc }}$ then we obtain a symmetric monoidal transformation

$$
H: \mathrm{Pic}_{\Omega A}^{\text {wloc,fl }} \rightarrow \mathrm{Pic}_{H \Omega A}^{\text {loc }}
$$

The de Rham equivalence is a canonical equivalence of sheaves

$$
H \Omega A \stackrel{\sim}{\rightarrow} \underline{H A}
$$


in $\mathrm{CAlg}\left(\operatorname{Mod}_{H \mathbb{R}}\right)$, see [Bun, Sec. 4.6].

Let us fix a commutative ring spectrum $R$ and a differential refinement $\hat{R}=(R, A, c)$. If we compose the inverse of the de Rham equivalence (12) with the sheafification of the equivalence $c: R \wedge H \mathbb{R} \rightarrow H A$ we obtain the equivalence

$$
\underline{R} \wedge H \mathbb{R} \stackrel{\sim}{\rightarrow} H \Omega A .
$$

in $\operatorname{CAlg}\left(\operatorname{Mod}_{H \mathbb{R}}\right)$. Using this equivalence we will consider $\underline{R} \wedge H \mathbb{R}$ as an object in Pic ${ }_{H \Omega A}^{\text {loc }}$.

Definition 4.9. A differential $R$-twist over a manifold $M$ is a triple $(E, \mathcal{M}, d)$ consisting of a topological $R$-twist $E \in \operatorname{Pic}_{R}^{\text {loc }}(M)$, an object $\mathcal{M} \in \operatorname{Pic}_{\Omega A}^{\text {wloc,fl }}(M)$, and an equivalence

$$
d: E \wedge H \mathbb{R} \stackrel{\sim}{\rightarrow} H \mathcal{M}
$$

in $\operatorname{Pic}_{H \Omega A}^{\mathrm{loc}}(M)$. We call the triple $(E, \mathcal{M}, d)$ a differential refinement of the topological twist E.

Definition 4.10. The $\infty$-stack of differential $R$-twists is defined by the pullback in $\mathrm{Sh}_{\mathrm{CGrp}(\mathcal{S})}$

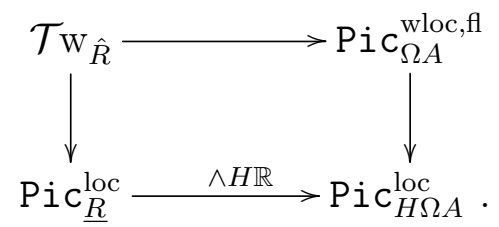

By construction, the objects of $\mathcal{T}_{\mathrm{w}_{\hat{R}}}(M)$ are differential $R$-twists over $M$.

Now let $(E, \mathcal{M}, d)$ be a differential twist of a differential spectrum $(R, A, c)$ over $M$. By $\mathcal{M}^{\geq 0}$ we denote the naive truncation of the sheaf of complexes to non-negative degrees. We have a canonical inclusion $i: \mathcal{M}^{\geq 0} \rightarrow \mathcal{M}$. Furthermore, let $\kappa: E \rightarrow E \wedge H \mathbb{R}$ be the canonical map.

Definition 4.11. The twisted differential function spectrum for a differential twist $(E, \mathcal{M}, d)$ is the sheaf of spectra defined by the pull-back in $\operatorname{Sh}_{\mathcal{S} \mathrm{p}}(M)$

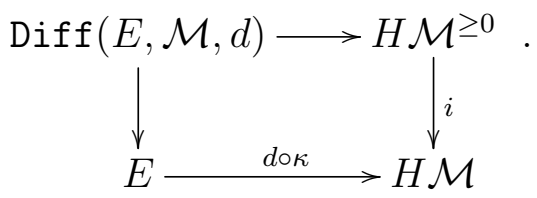

In the square above all objects are considered as sheaves of spectra, i.e. we omitted to write the forgetful functors $\operatorname{Mod}_{\underline{R}}(M) \rightarrow \operatorname{Sh}_{\mathcal{S}_{\mathrm{p}}}(M)$ and $\operatorname{Mod}_{H \Omega A} \rightarrow \mathrm{Sh}_{\mathcal{S}_{\mathrm{p}}}(M)$ explicitly.

Note that pullbacks in sheaves of spectra are computed objectwise, i.e. we get a similar pullback after evaluation at $M$ or more generally every manifold over $M$. In order to properly describe the functorial properties of the twisted differential function spectrum we use the same procedure as in the case of the classical twisted cohomology. Roughly speaking we consider the functor that assigns to a differential twist $(E, \mathcal{M}, d)$ over $M$ the spectrum $\operatorname{Diff}(E, \mathcal{M}, d)(M)$ thus defining a graded ring spectrum. 
Proposition 4.12. The construction 4.11 of the twisted differential function spectrum refines to a transformation

$$
\hat{\mathcal{R}} \in \operatorname{Sh}_{\mathrm{Gr} \mathcal{R} \operatorname{ing} \mathcal{S} \mathrm{p}}, \quad M \mapsto\left(\mathcal{T}_{\mathrm{w}_{\hat{R}}}(M), \operatorname{Diff}(\ldots)(M)\right)
$$

Proof. We again consider the functor $F$ introduced in (8). We set

$$
G:=\mathcal{T}_{\hat{R}} \in \operatorname{Sh}_{\mathcal{S y m}_{\mathcal{M}} \mathrm{on}_{\infty}}
$$

In order to apply Proposition A.5 we must construct a lax symmetric monoidal transformation

$$
\eta: \mathcal{T}_{\mathrm{W}} \rightarrow \mathrm{Sh}_{\mathcal{S} \mathrm{p}}
$$

such that $\eta(M)(E, \mathcal{M}, d) \cong \operatorname{Diff}(E, \mathcal{M}, d)$. Therefore let $J$ be the indexing category for pullback diagrams, i.e. $J=\Delta[1] \cup_{\Delta[0]} \Delta[1]$. The limit functor

$$
\lim _{J}: \mathrm{Sh}_{\mathcal{S} \mathrm{p}}^{J} \rightarrow \mathrm{Sh}_{\mathcal{S} \mathrm{p}}
$$

admits a lax symmetric monoidal refinement. This refinement is constructed using that $\lim _{J}$ is right adjoint to the diagonal functor which admits a canonical monoidal structure. Then we employ the fact that the right adjoint to a monoidal functor inherits a lax monoidal structure as shown in [Lur11, Corollary 8.3.2.7]. Thus in order to construct the transformation (14) it suffices to construct a symmetric monoidal transformation

$$
\mathcal{T}_{\mathrm{w}_{\hat{R}}} \rightarrow \mathrm{Sh}_{\mathcal{S} \mathrm{p}}^{J}
$$

By definition we have the following pullback decompositions:

$$
\begin{aligned}
& \mathcal{T}_{\mathrm{W}} \hat{R} \cong \mathrm{Pic}_{\Omega A}^{\text {wloc,fl }} \times_{\mathrm{Pic}_{H \Omega A}^{\text {loc }}} \mathrm{Pic}_{\underline{R}}^{\text {loc }} \\
& \operatorname{Sh}_{\mathcal{S} \mathrm{p}}^{J} \cong \operatorname{Sh}_{\mathcal{S}_{\mathrm{p}}}^{\Delta[1]} \times_{\mathrm{Sh}_{\mathcal{S}_{\mathrm{p}}}^{\Delta[0]}} \operatorname{Sh}_{\mathcal{S} \mathrm{p}}^{\Delta[1]}
\end{aligned}
$$

Therefore it suffices to write down a transformation between these diagrams, i.e. lax symmetric monoidal transformations

$$
f_{1}: \operatorname{Pic}_{\Omega A}^{\text {wloc,fl }} \rightarrow \operatorname{Sh}_{\mathcal{S} \mathrm{p}}^{\Delta[1]}, \quad f_{2}: \operatorname{Pic}_{\underline{R}}^{\text {loc }} \rightarrow \operatorname{Sh}_{\mathcal{S}_{\mathrm{p}}}^{\Delta[1]}, \quad f_{0}: \mathrm{Pic}_{H \Omega A}^{h} \rightarrow \mathrm{Sh}_{\mathcal{S}_{\mathrm{p}}}^{\Delta[0]}
$$

and fillers. The transformation

$$
f_{0}: \mathrm{Pic}_{H \Omega A}^{\text {loc }} \rightarrow \operatorname{Mod}_{H \Omega A} \rightarrow \mathrm{Sh}_{\mathcal{S} \mathrm{p}}
$$

is a composition of lax symmetric monoidal transformations.

$$
f_{1}(\mathcal{M}):=\left(H \mathcal{M}_{\geq 0} \rightarrow H \mathcal{M}\right), \quad \mathcal{M} \in \operatorname{Pic}_{\Omega A}^{\text {wloc,fl }}(M)
$$

and

$$
f_{2}(E):=(\kappa: E \rightarrow E \wedge H \mathbb{R}), \quad E \in \operatorname{Pic}_{\underline{R}}^{\text {loc }}(M),
$$

where we again omit two write various forgetful functors to $\mathrm{Sh}_{\mathcal{S}_{\mathrm{p}}}$. 
Definition 4.13. Given a manifold $M$ with a differential twist $(E, \mathcal{M}, d)$ we define the twisted differential cohomology group by

$$
\hat{R}^{(E, \mathcal{M}, d)}(M):=\pi_{0}(\operatorname{Diff}(E, \mathcal{M}, d)(M))
$$

Corollary 4.14. For each manifold $M$ the twisted differential cohomology $\hat{R}^{*}(M)$ forms a graded ring, which is graded over the homotopy category $\mathcal{H}_{\mathrm{o}} \mathcal{T}_{\mathrm{w}}(M)$. Moreover the assignment

$$
M \mapsto\left(\mathcal{H o}_{\mathrm{O}} \mathcal{W}_{\hat{R}}(M), \hat{R}^{*}(M)\right)
$$

forms a functor $\mathcal{M} \mathrm{f}^{o p} \rightarrow$ GrRing.

Theorem 4.15. The construction of the twisted differential function spectrum refines to a functor

$$
\widehat{\operatorname{Rings}} \rightarrow \operatorname{Sh}_{\mathrm{Gr} \mathcal{R} \text { ing } \mathcal{S}}
$$

Proof. The proof works similar to the proof of Proposition 3.8. We consider the functors

$$
F: \widehat{\operatorname{Rings}} \times \mathcal{M} \mathrm{f}^{o p} \stackrel{\mathrm{pr}_{2}}{\longrightarrow} \mathcal{M} \mathrm{f}^{o p} \stackrel{8}{\longrightarrow} \operatorname{Gr} \mathcal{R} \operatorname{ing} \mathcal{S} \mathrm{p}
$$

and

$$
G: \widehat{\operatorname{Rings}} \times \mathcal{M f}^{o p} \rightarrow \mathcal{S}_{\mathrm{ym}} \mathcal{M o n}_{\infty}, \quad(\hat{R}, M) \mapsto \mathcal{T}_{\mathrm{w}_{\hat{R}}}(M)
$$

and the lax symmetric monoidal transformation $\eta: G \rightarrow \mathrm{Sh}_{\mathcal{S} \mathrm{p}}$ constructed similar to the one in the proof of Propositon 4.12

\section{$5 \quad$ Properties of twisted differential cohomology}

In this section we list the basic properties of differential cohomology. The more advanced properties, such as orientation and integration theory will be discussed elsewhere.

The following list of structures just decodes the fact that Diff is a sheaf of graded ring spectra and correspond to similar structures in the topological case listed in Section 3. We fix a commutative ring spectrum $R$ and a differential refinement $\hat{R}$.

1. For every differential twist $(E, \mathcal{M}, d) \in \mathcal{H o}_{\mathrm{o}} \mathcal{T}_{\mathrm{R}}(M)$ we have an abelian group $\hat{R}^{(E, \mathcal{M}, d)}(M)$.

2. For every pair $(E, \mathcal{M}, d),\left(E^{\prime}, \mathcal{M}^{\prime}, d^{\prime}\right) \in \mathcal{H}_{\mathrm{o}} \mathcal{T}_{\mathrm{w}}(M)$ of differential twists we have a multiplication morphism

$$
\cup: \hat{R}^{(E, \mathcal{M}, d)}(M) \otimes \hat{R}^{\left(E^{\prime}, \mathcal{M}^{\prime}, d^{\prime}\right)}(M) \rightarrow \hat{R}^{(E, \mathcal{M}, d)+\left(E^{\prime}, \mathcal{M}^{\prime}, d^{\prime}\right)}(M),
$$

where $(E, \mathcal{M}, d)+\left(E^{\prime}, \mathcal{M}^{\prime}, d^{\prime}\right)$ denotes the symmetric monoidal pairing in $\mathcal{H o P i c}_{\hat{R}}(M)$. The multiplication is associative, commutative, and has a unit in the obvious sense. 
3. For every morphisms $(E, \mathcal{M}, d) \rightarrow\left(E^{\prime}, \mathcal{M}^{\prime}, d^{\prime}\right)$ in $\mathcal{H}_{\mathrm{o}} \mathcal{T}_{\mathrm{w}}(M)$ we have an isomorphism

$$
R^{(E, \mathcal{M}, d)}(M) \stackrel{\sim}{\rightarrow} R^{\left(E^{\prime}, \mathcal{M}^{\prime}, d^{\prime}\right)}(M) .
$$

This assignment is compatible with composition and the product in the obvious sense.

4. For every smooth map $f: M \rightarrow N$ and $(E, \mathcal{M}, d) \in \mathcal{H o P i c}_{\hat{R}}(N)$ we have a morphism

$$
R^{(E, \mathcal{M}, d)}(N) \rightarrow R^{f^{*}(E, \mathcal{M}, d)}(M)
$$

which is compatible with all the other maps.

The following structures are typical for differential cohomology and immediately follow from the definition of the twisted differential function spectrum by a pull-back. In the following proposition, if not said differently, $M$ is a smooth manifold and $(E, \mathcal{M}, d) \in$ $\mathcal{T}_{\mathrm{w}_{\hat{R}}}(M)$ is a differential twist.

Proposition 5.1. $\quad$ 1. We have a map of sheaves of graded rings spectra

$$
I: \widehat{\mathcal{R}} \rightarrow \mathcal{R} .
$$

It induces a map of abelian groups

$$
\widehat{R}^{(E, \mathcal{M}, d)} \rightarrow R^{E}
$$

which is compatible with the products, the action of morphisms between differential twists and smooth maps between manifolds in the natural sense.

2. We have a natural transformation

$$
\mathcal{C} \text { urv }: \widehat{R} \rightarrow \mathcal{Z},
$$

where the presheaf of graded rings $\mathcal{Z} \in \mathrm{PSh}_{\text {GrRing }}$ is given by

$$
M \mapsto\left(\operatorname{Pic}_{\Omega A}^{\text {wloc,fl }}(M) \in \mathcal{M} \mapsto \mathcal{Z}^{\mathcal{M}}(M):=Z^{0}(\mathcal{M}(M))\right) .
$$

It induces a map of abelian groups

$$
\mathcal{C} \text { urv : } \widehat{R}^{(E, \mathcal{M}, d)}(M) \rightarrow Z^{0}(\mathcal{M}(M))
$$

which is compatible with the product, the action of morphisms between differential twists and smooth maps between manifolds.

3. There is a canonical homomorphism

$$
a: \mathcal{M}^{-1}(M) / \operatorname{Im}(d) \rightarrow \widehat{R}^{(E, \mathcal{M}, d)}(M)
$$

which is natural in $M$ and such that it becomes a homomorphism of $\mathcal{T}_{\mathrm{w}_{\hat{R}}}(M)$-graded groups (not rings!). Moreover we have the rule

$$
a(\omega) \cup x=a(\omega \cup \mathcal{C} \operatorname{urv}(x))
$$

in $\hat{R}^{(E, \mathcal{M}, d)+\left(E^{\prime}, \mathcal{M}^{\prime}, d^{\prime}\right)}(M)$ for $\omega \in \mathcal{M}^{-1}(M) / \operatorname{Im}(d)$ and $x \in \widehat{R}^{\left(E^{\prime}, \mathcal{M}^{\prime}, d^{\prime}\right)}(M)$. 
4. The following diagram commutes:

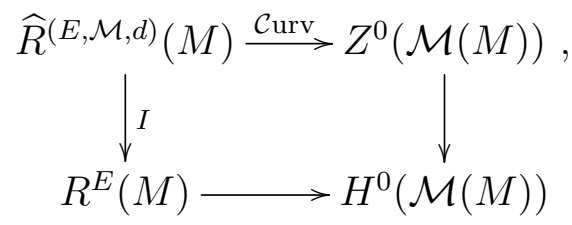

where the lower horizontal map is induced by the map $E \stackrel{\kappa}{\rightarrow} E \wedge H \mathbb{R} \stackrel{d}{\rightarrow} H \mathcal{M}$.

5. We have the equality

$$
\mathcal{C} \text { urv } \circ a=d
$$

of maps $\mathcal{M}^{-1}(M) / \operatorname{im}(d) \rightarrow Z^{0}(\mathcal{M}(M))$.

6. The following sequences are exact:

$$
\begin{array}{r}
R^{E-1}(M) \rightarrow \mathcal{M}^{-1}(M) / \operatorname{Im}(d) \rightarrow \widehat{R}^{(E, \mathcal{M}, d)}(M) \rightarrow R^{E}(M) \\
R^{E-1}(M) \rightarrow 0 \\
H^{-1}(\mathcal{M}(M)) \rightarrow \widehat{R}^{(E, \mathcal{M}, d)}(M) \rightarrow Z^{0}(\mathcal{M}(M)) \times_{H^{0}(\mathcal{M}(M))} R^{E}(M) \rightarrow 0
\end{array}
$$

Proof. This follows as in the untwisted case from the construction of $\widehat{R}^{(E, \mathcal{M}, d)}$ as a pullback. For more details we refer to the corresponding results in [BG13], [Bun].

The fact that $\widehat{R}^{(E, \mathcal{M}, d)}$ is a sheaf implies a Mayer-Vietoris sequence for twisted differential cohomology. By abuse of notation we denote $\pi_{0}$ of the evaluation of the sheaf $E \wedge M \mathbb{R} / \mathbb{Z}$ (which is not a twist) on $M$ by $R^{E \wedge M \mathbb{R} / \mathbb{Z}}(M)$, where $M \mathbb{R} / \mathbb{Z}$ is the Moore spectrum of the abelian group $\mathbb{R} / \mathbb{Z}$.

Proposition 5.2 (Mayer-Vietoris). Let $M=U \cup V$ be a decomposition of a smooth manifold as a union of two open subsets and $(E, \mathcal{M}, d) \in \mathcal{T}_{\mathrm{w}_{\hat{R}}}(M)$ be a differential twist. Then we have the long exact sequence

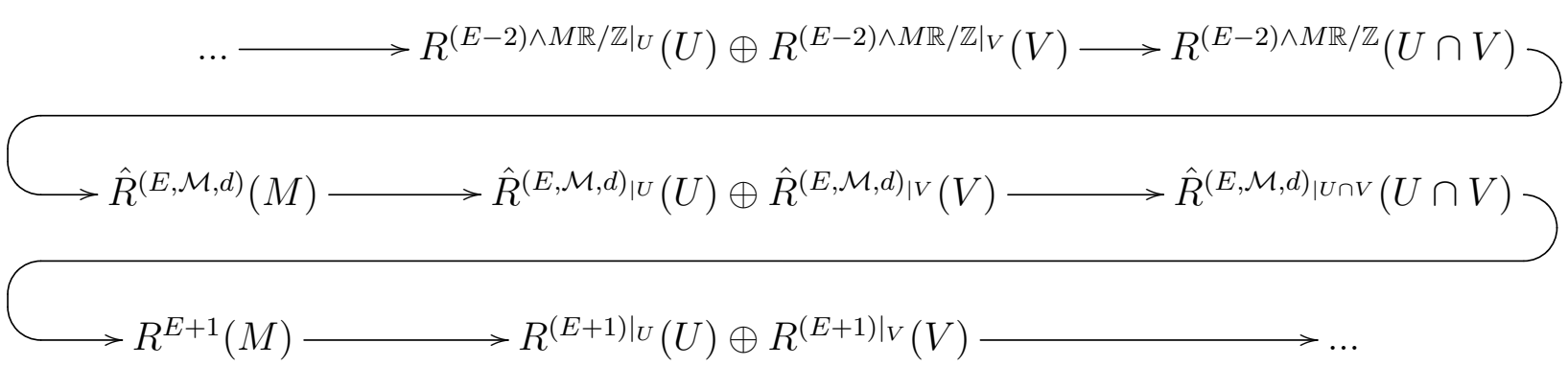

Finally we explain the consequence of the fact that the construction

$$
\widehat{\operatorname{Rings}} \ni \hat{R} \mapsto \widehat{\mathcal{R}} \in \mathrm{Sh}_{\text {GrRing } \mathcal{R}}
$$

is functorial. Let $f: R \rightarrow R^{\prime}$ be a morphism of commutative ring spectra. Assume that we have chosen a lift of this morphisms to differential refinements

$$
\hat{f}: \hat{R}=(R, A, c) \rightarrow\left(R^{\prime}, A^{\prime}, c^{\prime}\right)=\hat{R}^{\prime} .
$$


Then we have a morphism of sheaves of graded commutative ring spectra $\widehat{f}: \widehat{\mathcal{R}} \rightarrow \widehat{\mathcal{R}}^{\prime}$. Let us spell out some pieces of this structure. If $(E, \mathcal{M}, d) \in \mathcal{T}_{\mathrm{w}} \hat{R}$ is a differential twist of $\hat{R}$, then we get a differential twist $\widehat{f}_{*}(E, \mathcal{M}, d)=:\left(E^{\prime}, \mathcal{M}^{\prime}, d^{\prime}\right)$. In greater detail,

$$
E^{\prime} \cong E \wedge_{\underline{R}} \underline{R}^{\prime}, \quad \mathcal{M}^{\prime} \cong \mathcal{M} \otimes_{\Omega A} \Omega A^{\prime} .
$$

We further get an induced map

$$
\widehat{R}^{(E, \mathcal{M}, d)}(M) \rightarrow \widehat{R}^{\prime\left(E^{\prime}, \mathcal{M}^{\prime}, d^{\prime}\right)}(M)
$$

which is compatible with corresponding maps on the level of forms and underlying twisted cohomology classes. Furthermore, these maps preserve the products and are functorial and natural with respect to morphisms of differential twists and smooth manifolds. We refrain from writing out these details.

Example 5.3. Here is a typical example. We consider the Atiyah-Bott Shapiro orientation $f: M$ Spin $^{c} \rightarrow \mathrm{K}$. Since both spectra are formal we can take canonical differential refinements. We can further choose a differential ABS-orientation $\hat{f}: \widehat{\operatorname{Spin}^{c}} \rightarrow \widehat{\mathrm{K}}$. In this way we obtain a transformation

$$
\widehat{A}: \widehat{\mathcal{M S p i n}}^{c} \rightarrow \widehat{\mathcal{K}}
$$

of sheaves of graded ring spectra.

\section{Part II}

\section{De Rham models}

\section{Twisted de Rham complexes}

In Definition 4.9 we have introduced differential twists on a smooth manifold $M$ as triples $(E, \mathcal{M}, d)$ consisting of a topological twist $E$ together with a sheaf $\mathcal{M}$ of $K$-flat, invertible, and weakly locally constant DG- $\Omega A$-modules and an equivalence $d: E \wedge H \mathbb{R} \stackrel{\sim}{\rightarrow} H \mathcal{M}$ of $H \Omega A$-modules. In this section we show how examples of such sheaves $\mathcal{M}$ can be constructed from differential geometric data on $M$.

We consider a CDGA $A$. The following condition on $A$ will play an important role below.

Definition 6.1. The CDGA $A$ is called split if the differential $d: A^{-1} \rightarrow A^{0}$ vanishes.

By

$$
\operatorname{Pic}_{\underline{A}}^{\text {loc,fl }} \in \operatorname{Sh}_{\mathrm{CGrp}(\mathcal{S})}
$$

we denote the Picard-1 stack which associates to a manifold $M$ the groupoid $\mathrm{Pic}_{A}^{\text {loc,fl }}(M)$ of $K$-flat, locally constant sheaves of invertible $A$-modules on $\mathcal{M f} / M$. Our basic example of such a sheaf is $\underline{A}_{\mid M}$. 
Note that $\operatorname{Pic}_{\underline{A}}^{\text {loc,fl }}(M)$ is an ordinary groupoid. In the following we calculate the homotopy groups $\pi_{i}\left(\operatorname{Pic}_{A}^{\frac{1}{\text { loc,fl }}}(M)\right)$ for $i=0,1$. For a manifold $M$ let $\Pi_{1}(M)$ denote the fundamental groupoid of $M$. The objects are points in $M$, and the morphisms are homotopy classes of smooth pathsin $M$. By definition the Picard groupoid

$$
\operatorname{Pic}_{A}^{\mathrm{fl}}:=\operatorname{Pic}_{\underline{A}}^{\mathrm{loc}, \mathrm{fl}}(*)
$$

is the groupoid of $K$-flat invertible $A$-modules. Covering theory provides a natural identification

$$
\operatorname{Pic}_{\underline{A}}^{\text {loc,fl }}(M) \cong \operatorname{Fun}\left(\Pi_{1}(M), \operatorname{Pic}_{A}^{\mathrm{fl}}\right) \text {. }
$$

For every $X \in \mathrm{Pic}_{A}^{\mathrm{fl}}$ we have a canonical identification of abelian groups

$$
\operatorname{Aut}_{\operatorname{Pic}_{A}^{\mathrm{f}}}(X) \cong Z^{0}(A)^{\times} .
$$

To see this first of all note that the action of $A$ on $X$ provides an identification $A^{0} \cong$ $\operatorname{End}_{A^{\sharp}}\left(X^{\sharp}\right)$, where $(\cdots)^{\sharp}$ indicates the operation of forgetting the differential. The condition that $a \in A$ preserves the differential translates to $a \in Z^{0}(A)$. Finally, if $a$ induces an isomorphism, then $a \in Z^{0}(A)^{\times}$.

Proposition 6.2. If $M$ is a connected smooth manifold with a given base point $m \in M$, then we have natural isomorphisms

$$
\pi_{0}\left(\operatorname{Pic}_{\underline{\underline{A}}}^{\text {loc,fl }}(M)\right) \cong \pi_{0}\left(\operatorname{Pic}_{A}^{\mathrm{A}}\right) \oplus H^{1}\left(M ; Z^{0}(A)^{\times}\right)
$$

and

$$
\pi_{1}\left(\operatorname{Pic}_{\underline{A}}^{\text {loc,fl }}(M), X\right) \cong Z^{0}(A)^{\times}
$$

for all $X \in \operatorname{Pic}_{\underline{A}}^{\text {loc,fl }}(M)$.

For a sheaf $\mathcal{A} \in \operatorname{Pic}_{\underline{A}}^{\text {loc, }, \mathrm{f}}(M)$ we define the sheaf of DG- $\Omega A_{\mid M}$-modules

$$
\mathcal{M}(\mathcal{A}):=\Omega_{\mid M} \otimes_{\mathbb{R}} \mathcal{A} \in \operatorname{Mod}_{\Omega A}(M) .
$$

Lemma 6.3. We have $\mathcal{M}(\mathcal{A}) \in \operatorname{Pic}_{\Omega A}^{\text {wloc,fl}}(M)$.

Proof. We must show that $\mathcal{M}(\mathcal{A})$ is

1. $K$-flat,

2. invertible,

3. and weakly locally constant. 
1. We have a canonical isomorphism $\mathcal{M}(\mathcal{A}) \cong \Omega A_{\mid M} \otimes_{A} \mathcal{A}$. If $X \rightarrow Y$ is a quasiisomorphism in $\operatorname{Mod}_{\Omega A}(M)$, then we can identify the induced morphism $\mathcal{M}(\mathcal{A}) \otimes_{\Omega A_{\mid M}} X \rightarrow$ $\mathcal{M}(\mathcal{A}) \otimes_{\Omega A_{\mid M}} Y$ with $\mathcal{A} \otimes_{A} X \rightarrow \mathcal{A} \otimes_{A} Y$. Here we consider $X$ and $Y$ as sheaves of $A$ modules by restriction along the inclusion $\underline{A} \mapsto \Omega A_{\mid M}$ This shows that $K$-flatness of $\mathcal{A}$ implies $K$-flatness of $\mathcal{M}(\mathcal{A})$.

2. If $\mathcal{A} \in \mathrm{Pic}_{A}^{\text {loc,fl }}(M)$, then there exists $\mathcal{B} \in \mathrm{Pic}_{\underline{A}}^{\text {loc,fl }}(M)$ such that $\mathcal{A} \otimes_{A} \mathcal{B} \cong \underline{A}_{\mid M}$. We see that then $\mathcal{M}(\mathcal{A}) \otimes_{\Omega A_{\mid M}} \mathcal{M}(\mathcal{B}) \cong \Omega A_{\mid M}$. Hence $\mathcal{M}(\mathcal{A})$ is invertible.

3. Let $U \subseteq M$ be such that $\mathcal{A}_{\mid U} \cong \underline{X}_{\mid U}$ for some $K$-flat invertible $A$-module $X$. Then we have $\mathcal{M}(\mathcal{A})_{\mid U} \cong \Omega A_{\mid U} \otimes_{A} \underline{X}_{\mid U} \cong \Omega_{\mid U} \otimes_{\mathbb{R}} \underline{X}_{\mid U}$. The equivalence $\iota(\mathbb{R}) \stackrel{\sim}{\rightarrow} \iota(\Omega)$ induces the equivalence $\underline{\iota(X)}_{\mid U} \stackrel{\sim}{\rightarrow} \iota\left(\Omega_{\mid U} \otimes_{\mathbb{R}} \underline{X}_{\mid U}\right)$.

We thus get a symmetric monoidal transformation of Picard stacks

$$
\mathcal{M}: \mathrm{Pic}_{\underline{A}}^{\text {loc,fl }} \rightarrow \mathrm{Pic}_{\Omega A}^{\text {wloc,fl }}
$$

We consider a manifold $M$ and an object $\mathcal{A} \in \mathrm{Pic}_{A}^{\text {loc,fl }}(M)$. We use the symbol $d$ in order to denote the differentials of $\Omega A$ and of $\mathcal{M}(\mathcal{A})$. An element $\omega \in \Omega A(M)$ induces a multiplication map $\omega: \mathcal{M}(\mathcal{A})^{\sharp} \rightarrow \mathcal{M}(\mathcal{A})^{\sharp}$ of modules over the sheaf of graded commutative algebra $\Omega A_{\mid M}^{\sharp}$ (recall that $\sharp$ stands for forgetting differentials). Furthermore we have the identity $d \omega=[d, \omega]$. If $\omega \in \Omega A^{1}(M)$, then $\omega^{2}=0$, and if $\omega$ is in addition closed, then $(d+\omega)^{2}=d^{2}+[d, \omega]+\omega^{2}=0$. Hence a cycle

$$
\omega \in Z^{1}(\Omega A(M))
$$

can be used to deform this differential $d$ to $d+\omega$.

Definition 6.4. We let $\mathcal{M}(\mathcal{A}, \omega) \in \operatorname{Mod}_{\Omega A}(M)$ denote the sheaf of $\Omega A_{\mid M}$-modules $\mathcal{M}(\mathcal{A})$ with new differential $d+\omega$.

More precisely, if $f: N \rightarrow M$ is an object of $\mathcal{M f} / M$, then $\mathcal{M}(\mathcal{A}, \omega)(N \rightarrow M)$ is the complex $\mathcal{M}(\mathcal{A})(N \rightarrow M)$ with the new differential $d+f^{*} \omega$.

In the following we analyse when $\mathcal{M}(\mathcal{A}, \omega)$ is invertible, weakly locally constant, and $K$-flat.

Lemma 6.5. Let $\mathcal{A} \in \mathrm{Pic}_{\underline{A}}^{\mathrm{loc}, \mathrm{fl}}(M)$ and $\omega \in Z^{1}(\Omega A(M))$. Then $\mathcal{M}(\mathcal{A}, \omega)$ is invertible.

Proof. For $\mathcal{A}, \mathcal{A}^{\prime} \in \mathrm{Pic}_{A}^{\text {loc,fl }}(M)$ and $\omega, \omega^{\prime} \in Z^{1}(\Omega A(M))$ we have the rule

$$
\mathcal{M}(\mathcal{A}, \omega) \otimes_{\Omega A_{\mid M}} \mathcal{M}\left(\mathcal{A}^{\prime}, \omega^{\prime}\right)=\mathcal{M}\left(\mathcal{A} \otimes_{A} \mathcal{A}^{\prime}, \omega+\omega^{\prime}\right)
$$


Hence if $\mathcal{B} \in \operatorname{Pic}_{\underline{A}}^{\text {loc,fl }}(M)$ is such that $\mathcal{A} \otimes_{A} \mathcal{B} \cong \underline{A}_{\mid M}$, then by 15 we get

$$
\mathcal{M}(\mathcal{A}, \omega) \otimes_{\Omega A_{\mid M}} \mathcal{M}(\mathcal{B},-\omega) \cong \Omega A_{\mid M}
$$

Let $\beta \in F^{1} \Omega A^{0}(M)$, where $F^{p} \Omega A(M) \subseteq \Omega A(M)$ denotes the subcomplex of forms with differential form degree $\geq p$. Then $\beta$ is nilpotent and the multiplication by $\exp (\beta)$ provides an isomorphism

$$
\exp (\beta): \mathcal{M}(\mathcal{A}, \omega+d \beta) \stackrel{\sim}{\rightarrow} \mathcal{M}(\mathcal{A}, \omega)
$$

Lemma 6.6. We assume that $A$ is split. Let $\mathcal{A} \in \mathrm{Pic}_{A}^{\text {loc,fl }}(M)$ and $\omega \in Z^{1}\left(F^{2} \Omega A(M)\right)$. Then the $\Omega A_{\mid M}$-module $\mathcal{M}(\mathcal{A}, \omega)$ is $K$-flat and weakly locally constant.

Proof. $K$-flatness and the condition of being weakly locally constant can be checked locally. Let $[\omega]$ denote the cohomology class of the cycle $\omega$. If $m \in M$, then $[\omega]_{\mid m}=\left[\omega_{\mid m}\right]=$ 0 by the filtration condition. Let $U \subseteq M$ be a contractible neighbourhood of $m$. Since the cohomology of $\Omega A$ is homotopy invariant, we get that $\omega_{\mid U}=d \beta$ for some $\beta \in \Omega A^{0}(U)$. Let us write $\beta=\sum_{i \in \mathbb{N}} \beta^{i}$, where $\beta^{i} \in\left(\Omega^{i} \otimes A^{-i}\right)(U)$ and set $\beta^{\geq 1}:=\sum_{i=1}^{\infty} \beta^{i}$. Since $A$ is split we still have $d \beta^{\geq 1}=\omega_{\mid U}$. We get an isomorphism $\exp (-\beta): \mathcal{M}(\mathcal{A}, \omega)_{\mid U} \cong \mathcal{M}(\mathcal{A})_{\mid U}$ in $\operatorname{Mod}_{\Omega A}(U)$. Since $\mathcal{M}(\mathcal{A})_{\mid U}$ is $K$-flat and weakly locally constant by Lemma 6.3 , so is $\mathcal{M}(\mathcal{A}, \omega)_{\mid U}$.

A homomorphism of abelian groups $d: X \rightarrow Y$ defines a Picard groupoid with objects $Y$ and morphisms $x: y \rightarrow y^{\prime}$ for $y, y^{\prime} \in Y$ and $x \in X$ with $y+d x=y^{\prime}$.

Remark 6.7. As Picard- $\infty$-groupoid it is equivalent to $\Omega^{\infty} H(X \rightarrow Y)$, where we consider $X \rightarrow Y$ as a chain complex with $Y$ in degree 0 .

In order to make the following definition we assume that $A$ is split.

Definition 6.8. We let $\mathbf{Z}_{A}^{1} \in \mathrm{Sh}_{\mathrm{CGrp}(\mathcal{S})}$ denote the Picard- $\infty$ stack induced by the map

$$
F^{1} \Omega A^{0} \stackrel{d}{\rightarrow} Z^{1}\left(F^{2} \Omega A\right)
$$

of sheaves of abelian groups.

Indeed, the condition that $A$ is split ensures that $d$ increases the filtration.

Proposition 6.9. Assume that $A$ is split. Then the assignment $(\mathcal{A}, \omega) \mapsto \mathcal{M}(\mathcal{A}, \omega)$ refines to a faithful transformation

$$
\mathcal{M}: \mathrm{Pic}_{\underline{A}}^{\text {loc,fl }} \times \mathbf{Z}_{A}^{1} \rightarrow \mathrm{Pic}_{\Omega A}^{\text {wloc,fl }}
$$

between symmetric monoidal stacks 
Proof. The formula in the statement of the proposition describe the action of the functor on objects. Let $(\phi, \beta):(\mathcal{A}, \omega) \rightarrow\left(\mathcal{A}^{\prime}, \omega^{\prime}\right)$ be a morphism in $\left(\mathrm{Pic}_{\underline{A}}^{\text {loc,fl }} \times \mathbf{Z}_{A}^{1}\right)(M)$. Then $\mathcal{M}(\phi, \beta): \mathcal{M}(\mathcal{A}, \omega) \rightarrow \mathcal{M}\left(\mathcal{A}^{\prime}, \omega^{\prime}\right)$ is given by

$$
\mathcal{M}(\mathcal{A}, \omega) \stackrel{i \mathrm{~d}_{\Omega A_{\mid M}} \otimes \phi}{\longrightarrow} \mathcal{M}\left(\mathcal{A}^{\prime}, \omega\right) \stackrel{\exp (\beta)}{\longrightarrow} \mathcal{M}\left(\mathcal{A}^{\prime}, \omega^{\prime}\right)
$$

where $\omega^{\prime}=\omega+d \beta$. The symmetric monoidal structure on $\mathcal{M}$ given by the canonical maps.

Finally we must show that $\mathcal{M}$ is faithful. Assume that

$$
\mathcal{M}(\phi, \beta)=\mathcal{M}\left(\phi^{\prime}, \beta^{\prime}\right)
$$

We conclude that $\phi=\phi^{\prime}$ by considering the restriction of the equality (19) to all points $m \in M$. It remains to show that $\beta=\beta^{\prime}$.

The ring of endomorphisms of an object in $\mathrm{Pic}_{\Omega A}^{\text {loc,fl }}(M)$ is naturally isomorphic to the ring $Z^{0}(\Omega A(M))$. The automorphism $\mathcal{M}\left(\phi, \beta^{\prime}\right)^{-1} \circ \mathcal{M}(\phi, \beta)=\mathcal{M}\left(\mathrm{id}, \beta-\beta^{\prime}\right)$ is given by multiplication with the cycle $\exp \left(\beta-\beta^{\prime}\right) \in 1+F^{1} \Omega A^{0}(M)$. The inverse of the exponential is given by the logarithm

$$
\log : 1+F^{1} \Omega A^{0}(M) \rightarrow F^{1} \Omega A^{0}(M), \quad \log (\gamma):=-\sum_{n=1}^{\infty} \frac{(\gamma-1)^{n}}{n}
$$

Hence we can recover the difference $\beta-\beta^{\prime}$ from the composition $\mathcal{M}\left(\phi, \beta^{\prime}\right)^{-1} \circ \mathcal{M}(\phi, \beta)$. If $\mathcal{M}\left(\phi, \beta^{\prime}\right)^{-1} \circ \mathcal{M}(\phi, \beta)=$ id, then $\beta=\beta^{\prime}$.

Let $k \in \mathbb{N}$ and $\omega \in Z^{1}\left(F^{1} \Omega A\left(S^{k+1}\right)\right)$. The sheaf $\mathcal{M}(\underline{A}, \omega) \in \operatorname{Pic}_{\Omega A}^{\text {wloc,fl }}\left(S^{k+1}\right)$ gives rise to an object $H \mathcal{M}(\underline{A}, \omega) \in \operatorname{Pic}_{\underline{H A}}^{\text {loc }}\left(S^{k+1}\right)$. In the following we calculate the invariant

$$
\nu(H \mathcal{M}(\underline{A}, \omega)) \in \pi_{k}(H A) \cong \widetilde{H A}^{0}\left(S^{k}\right)
$$

defined in Definition 3.13 . Here and below ${ }^{\sim}$ indicates reduced cohomology.

The composition of the de Rham equivalence with the suspension isomorphism provides an isomorphism

$$
\sigma: \tilde{H}^{1}\left(\Omega A\left(S^{k+1}\right)\right) \stackrel{\sim}{\rightarrow} \widetilde{H A}^{1}\left(S^{k+1}\right) \stackrel{\sim}{\rightarrow} \widetilde{H A}^{0}\left(S^{k}\right) .
$$

Lemma 6.10. We assume that $A$ is split. Then we have $\nu(H \mathcal{M}(\underline{A}, \omega))=\sigma([\omega])$.

Proof. We use the notation introduced in order to state Definition 3.13. We can find $\beta_{ \pm} \in \Omega A^{0}\left(D_{ \pm}\right)$with $d \beta_{ \pm}=\omega_{\mid D_{ \pm}}$. We can normalize $\beta$ such that $\beta_{ \pm \mid p}=0$. Since $A$ is split, we then, as in the proof of Lemma 6.6 or Lemma 7.2, can modify $\beta$ further such that $\beta_{ \pm} \in F^{1} \Omega A^{0}\left(D_{ \pm}\right)$. Then by the usual description of the suspension isomorphism in de Rham cohomology we have

$$
\sigma([\omega])=\left[\beta_{+, \mid S^{k}}-\beta_{-, \mid S^{k}}\right] \in \widetilde{H}^{0}\left(\Omega A\left(S^{k}\right)\right) \cong \widetilde{H A}^{0}\left(S^{k}\right) .
$$


The trivializations $\phi_{ \pm}: \mathcal{M}(\underline{A}, \omega)_{\mid D_{ \pm}} \stackrel{\sim}{\rightarrow} \Omega A_{\mid D_{ \pm}}$are now given by $\phi_{ \pm}:=\exp \left(-\beta_{ \pm}\right)$. According to Definition 3.13 we have

$$
\nu(H \mathcal{M}(\underline{A}, \omega))=\left[\exp \left(\beta_{+, \mid S^{k}}-\beta_{-, \mid S^{k}}\right)\right]-1=\left[\beta_{+, \mid S^{k}}-\beta_{-, \mid S^{k}}\right] .
$$

\section{Uniqueness of differential refinements of twists}

Let $(R, A, c)$ be a differential refinement (Definition 4.1) of a commutative ring spectrum $R$. In this section we show the following theorem.

Theorem 7.1. Let $M$ be a smooth manifold and assume that $A$ is split. Then the forgetful functor which projects to the underlying topological twist induces an injective homomorphism

$$
\pi_{0}\left(\mathcal{T}_{\mathrm{w}_{\hat{R}}}(M)\right) \rightarrow \pi_{0}\left(\operatorname{Pic}_{\underline{R}}^{\text {loc }}(M)\right) .
$$

In particular, if a R-twist on $M$ admits a differential refinement, then this refinement is unique up to (non-canonical) equivalence.

The proof of this theorem will be finished after some preparations at the end of this section.

Lemma 7.2. If $A$ is split, then

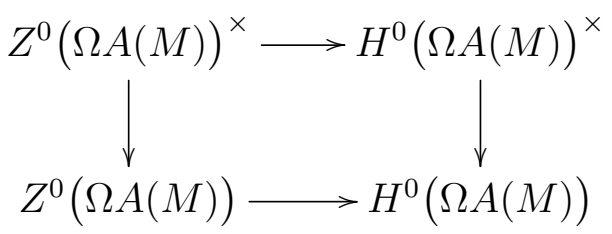

is a pullback diagram.

Proof. We first observe that it suffices to show the assertion under the additional assumption that $M$ is connected.

We consider a cycle $\omega \in Z^{0}(\Omega A(M))$ such that $[\omega] \in H^{0}(\Omega A(M))^{\times}$. We then must show that $\omega \in Z^{0}(\Omega A(M))^{\times}$.

We pick a point $m \in M$. Then $[\omega]_{\mid m} \in H^{0}(A)^{\times}$. We write $\omega=\sum_{i \in \mathbb{N}} \omega^{i}$, where $\omega^{i} \in\left(\Omega^{i} \otimes A^{-i}\right)(M)$. Since $A$ is split we have $d_{d R} \omega^{0}=0$ and therefore $\omega^{0}=1 \otimes x$, where $x \in Z^{0}(A)$ represents $[\omega]_{\mid m} \in H^{0}(A)$. Since $A$ is split we have $Z^{0}(A) \cong H^{0}(A)$. Since $[\omega]_{\mid m}$ is a unit we conclude that $x$ is a unit. This implies that $\omega$ is a unit, since $\omega-\omega^{0}$ is nilpotent. 
Lemma 7.3. We consider an object $Y \in \mathrm{Pic}_{\Omega A}^{\mathrm{wloc}, \mathrm{fl}}(M)$. Then we have an isomorphism

$$
\pi_{0}\left(\operatorname{Map}_{\operatorname{Mod}_{\iota(\Omega A)}(M)}\left(\iota(\Omega A)_{\mid M}, \iota(Y)\right)\right) \cong H^{0}(Y(M))
$$

Proof. The evaluation at $M$ fits into an adjunction

$$
\mathcal{F}: \mathrm{Ch}_{\infty} \leftrightarrows \operatorname{Mod}_{\iota(\Omega A)(M)} \text { : evaluation at } M
$$

such that $\mathcal{F}(\iota(\mathbb{Z}))=\iota(\Omega A)_{\mid M}$. This gives the equivalence

$$
\operatorname{Map}_{\operatorname{Mod}_{\iota(\Omega A)}(M)}\left(\iota(\Omega A)_{\mid M}, \iota(Y)\right) \cong \operatorname{Map}_{\mathrm{Ch}_{\infty}}(\iota(\mathbb{Z}), \iota(Y(M))) .
$$

We now use the fact that $\pi_{0}\left(\operatorname{Map}_{\mathrm{Ch}_{\infty}}(\iota(\mathbb{Z}), \iota(Y(M)))\right) \cong H^{0}(Y(M))$.

In order to formulate the following Lemma we use the fact that $\operatorname{Mod}_{\Omega A}(M)$ has a natural enrichment to a $D G$-category. In particular the notion of chain homotpy is well defined in $\operatorname{Mod}_{\Omega A}(M)$.

Lemma 7.4. Assume that $X, Y \in \operatorname{Pic}_{\Omega A}^{\text {wloc,fl}}(M)$. Then the following assertions hold true:

1. The map

$$
\operatorname{Hom}_{\operatorname{Mod}_{\Omega A}(M)}(X, Y) \rightarrow \pi_{0}\left(\operatorname{Map}_{\operatorname{Mod}_{\iota(\Omega A)}(M)}(\iota(X), \iota(Y))\right)
$$

is surjective.

2. If $f, f^{\prime} \in \operatorname{Hom}_{\operatorname{Mod}_{\Omega A}(M)}(X, Y)$ are equivalent in $\operatorname{Mod}_{\iota(\Omega A)}(M)$, i.e. they induce the same point in $\pi_{0}\left(\operatorname{Map}_{\operatorname{Mod}_{\iota(\Omega A)}(M)}(\iota(X), \iota(Y))\right)$, then $f$ and $f^{\prime}$ are chain homotopic.

Proof. 1. We first assume that $X=\Omega A_{\mid M}$. Then we have the isomorphisms

$$
\operatorname{Hom}_{\operatorname{Mod}_{\Omega A}(M)}\left(\Omega A_{\mid M}, Y\right) \cong Z^{0}(Y(M)) .
$$

Furthermore, we have the isomorphism

$$
\pi_{0}\left(\operatorname{Map}_{\operatorname{Mod}_{\iota(\Omega A)}(M)}\left(\iota(\Omega A)_{\mid M}, \iota(Y)\right)\right) \stackrel{\text { Lemmd }}{\cong} H^{0}(Y(M))
$$

The assertion now follows from the fact that the projection $Z^{0}(Y(M)) \rightarrow H^{0}(Y(M))$ is surjective.

In the general case we have the following commuting diagram

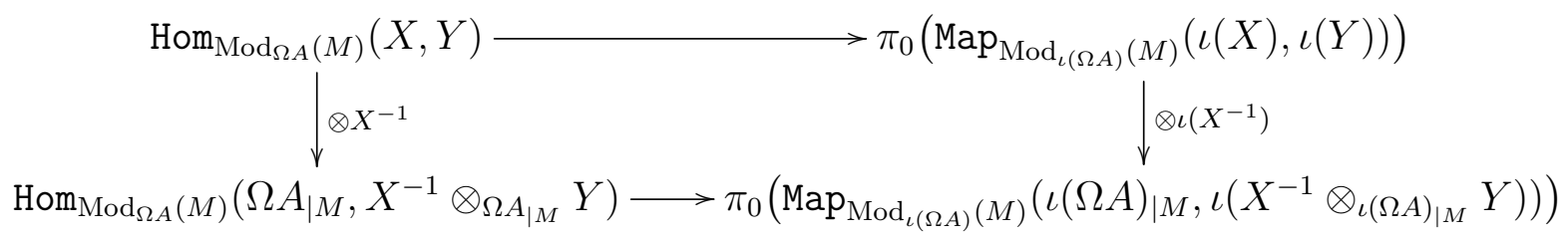


The vertical maps are both bijective. Hence the upper horizontal map is surjective since the lower one is.

2. For the second assertion, as for 1 ., we can reduce to the case where $X=\Omega A_{\mid M}$. In this case the map (20) reduces to the projection $Z^{0}(Y(M)) \rightarrow H^{0}(Y(M))$ and the assertion is obvious.

Proposition 7.5. If $A$ is split, then the canonical functor

$$
\iota: \operatorname{Pic}_{\Omega A}^{\text {wloc,fl }}(M) \rightarrow \mathcal{H} o \mathrm{Pic}_{\iota(\Omega A)}^{\text {loc }}(M)
$$

is full.

Proof. Assume we are given objects $X, Y$ in $\operatorname{Pic}_{\Omega A}^{\text {wloc,fl }}(M)$ and a morphism $F: \iota(X) \rightarrow$ $\iota(Y)$ in the homotopy category $\mathcal{H}_{\mathrm{oPi}} \mathrm{c}_{\iota(\Omega A)}^{\text {loc }}(M)$. By Lemma 7.4 (1) we can conclude that there is a morphism $f: X \rightarrow Y$ in $\operatorname{Mod}_{\Omega A}(M)$ such that $\iota(f)=F$. It remains to show that $f$ is an isomorphism.

Since $F$ is invertible there exists an inverse $G: \iota(Y) \rightarrow \iota(X)$ in $\mathcal{H} \circ \mathrm{Pic}_{\iota(\Omega A)}^{\text {loc }}(M)$, i.e. $G \circ F$ and $F \circ G$ are equivalent to the identities. By the first assertion of Lemma 7.4 there exists a morphism $g: Y \rightarrow X$ such that $\iota(g)=G$. By the second assertion of the Lemma the compositions $g \circ f$ and $f \circ g$ are chain-homotopic to the corresponding identities.

We can identify $\operatorname{Hom}_{\operatorname{Mod}_{\Omega A}(M)}(X, X) \cong Z^{0}(\Omega A(M))$ as a ring and $H^{0}(\Omega A(M))$ with chainhomotopy equivalence classes of endomorphisms such that multiplication corresponds to composition. Since the chain homotopy class of $g \circ f$ is invertible, it follows from Lemma 7.2 that $g \circ f$ itself is invertible. Similarly we see that $f \circ g$ is invertible. This implies that $f$ is an isomorphism.

Remark 7.6. More generally, the assertion of Proposition 7.5 holds true for $K$-flat invertible $\Omega A$-modules, i.e. the assumption of being weakly locally constant can be dropped.

Proof. (of Theorem 7.1): By Proposition 7.5 and the long exact sequence in homotopy the fibre of

$$
\operatorname{Pic}_{\Omega A}^{\text {wloc,fl }}(M) \stackrel{\iota}{\rightarrow} \operatorname{Pic}_{\iota(\Omega A)}^{\text {loc }}(M) \cong \operatorname{Pic}_{H \Omega A}^{\text {loc }}(M)
$$

is connected. By the Definition 13 of $\mathcal{T}_{\mathrm{w}}(M)$ as a pull-back the fibre of the map

$$
\mathcal{T}_{\mathrm{w}_{\hat{R}}}(M) \rightarrow \mathrm{Pic}_{\underline{R}}^{\mathrm{loc}}(M)
$$

is connected, too. 


\section{Existence of differential refinements of twists}

We consider a differential refinement $(R, A, c)$ of a commutative ring spectrum $R$ (Definition 4.1). In the statement of the following theorem we use the fact that if $W$ is an $R$-module spectrum, then its realification $W \wedge H \mathbb{R}$ has the strucure of an $H A$-module via $c$. In this section we show the following theorem.

Theorem 8.1. We assume that the CDGA $A$ is split. Let $M$ be a connected smooth manifold with a base point $m \in M$ and $E$ be a topological $R$-twist on $M$. Then the twist $E$ admits a differential refinement $(E, \mathcal{M}, d)$ if and only if there exists an invertible $K$ flat DG-A-module $X$ such that there exists an equivalence $E(\{m\}) \wedge H \mathbb{R} \cong H X$ of $H A$ modules.

Remark 8.2. Note that the verification of the condition in the theorem boils down to check whether the spectrum $E(\{m\}) \wedge H \mathbb{R}$ allows a real chain complex model. In fact, one can always find a DG- $A$-module which models this spectrum. But we require in addition that this DG- $A$-module is strictly invertible and $K$-flat, which is not automatic.

The following twists satisfy the condition:

1. shifts of the spectrum $R$;

2. twists classified by a map to $\operatorname{BGL}_{1}(R)$;

3. twists that are pointwise of the form of a shift of $R$. These are exactly the twists classified by a map to the spectrum of periodic units considered by Sagave Sag11.

The proof of this theorem requires some work and will be finished at the end of this section. The main point is to find the corresponding object $\mathcal{M}$ in $\operatorname{Pic}_{\Omega A}^{\text {wloc,fl }}(M)$. The idea is to take, as a first approximation, the sheaf $\mathcal{M}(\underline{X})$, where $X$ is the DG-A-module realizing the twist over the basepoint $m \in M$. This approximation is, by definition, correct at the base point $m$. In a second step we try to modify this sheaf $\mathcal{M}(\underline{X})$ by tensoring with a sheaf of the form $\mathcal{M}(\mathcal{A}, \omega)$ where $\mathcal{A} \in \operatorname{Pic}_{\underline{A}}^{\text {loc,fl }}(M)$ satisfies $\mathcal{A}_{\mid m} \cong A$. In order to ensure the existence of this correction we must understand the map

$$
\pi_{0}(H \mathcal{M}(-)): \pi_{0}\left(\operatorname{Pic}_{\underline{A}}^{\text {loc,fl }}(M) \times \mathbf{Z}_{A}^{1}(M)\right) \rightarrow \pi_{0}\left(\operatorname{Pic}_{\underline{H A}}^{\text {loc }}(M)\right)
$$

in some detail. The calculation of this map is stated in Corollary 8.12 .

We consider a spectrum $p$. For every topological space $X$ we have the Atiyah-Hirzebruch filtration

$$
\cdots \subseteq F^{2} p^{*}(X) \subseteq F^{1} p^{*}(X) \subseteq F^{0} p^{*}(X)=p^{*}(X)
$$

of the $p$-cohomology groups. By definition, for $k \in \mathbb{N}$ a class $c \in p^{*}(X)$ belongs to $F^{k} p^{*}(X)$ if and only if $f^{*} c=0$ for every map $f: Y \rightarrow X$ from a $k$-1-dimensional complex $Y$. The associated graded group $\operatorname{Gr} p^{*}(X)$ is calculated by the Atiyah-Hirzebruch spectral sequence (AHSS). If $p$ is a commutative ring spectrum, then the Atiyah-Hirzebruch filtration turns the cohomology $p^{*}(X)$ into a filtered graded commutative ring. 
For $k \in \mathbb{Z}$ we let $p\langle k\rangle \rightarrow p$ denote the $k$-connected covering of $P$. We have $\pi_{i}(p\langle k\rangle)=0$ for $i \leq k$ and an isomorphism $\pi_{i}(p\langle k\rangle) \stackrel{\sim}{\rightarrow} \pi_{i}(p)$ for all $i>k$. The following assertions are checked easily using the naturality of the AHSS. If $X$ is a space, then the natural map $p\langle 0\rangle \rightarrow p$ induces isomorphisms

$$
p\langle 0\rangle^{0}(X) \stackrel{\sim}{\rightarrow} F^{1} p^{0}(X), \quad p\langle 0\rangle^{1}(X) \stackrel{\sim}{\rightarrow} F^{2} p^{1}(X) .
$$

Similarly, the maps $p\langle 1\rangle \rightarrow p$ and $p\langle-1\rangle \rightarrow p$ induce isomorphisms

$$
p\langle 1\rangle^{0}(X) \stackrel{\sim}{\rightarrow} F^{2} p^{0}(X), \quad p\langle-1\rangle^{1}(X) \stackrel{\sim}{\rightarrow} F^{1} p^{1}(X) .
$$

The sheaf of CDGAs $\Omega A$ has a decreasing filtration

$$
\cdots \subseteq F^{2} \Omega A \subseteq F^{1} \Omega A \subseteq F^{0} \Omega A=\Omega A
$$

where $F^{k} \Omega A:=\prod_{i \geq k} \Omega^{i} \otimes A$. For every manifold $M$ we obtain an induced filtration on the cohomology groups $H^{*}(\Omega A(M))$. Recall that we have the de Rham equivalence (12).

Lemma 8.3. The de Rham equivalence induces is an isomorphism of filtered commutative graded algebras

$$
H^{*}(\Omega A(M)) \stackrel{\sim}{\rightarrow} H A^{*}(M) .
$$

Proof. The main new point is that the de Rham equivalence preserves the filtration. This will be shown in Lemma 12.1 in a more general situation.

Let $p$ be a spectrum and $P:=\Omega^{\infty} p \in \operatorname{CGrp}(\mathcal{S})$ be its infinite loop space. Then we obtain a constant Picard- $\infty$ stack $\underline{P} \in \operatorname{Sh}_{\mathrm{CGrp}(\mathcal{S})}$, see Definition 3.2. Note that

$$
\pi_{i}(\underline{P}(M)) \cong p^{-i}(M)
$$

for all $i \geq 0$. Recall Definition (7) of the Picard- $\infty$ stack Pic $\underline{R}_{\underline{R}}^{\text {loc }}$. We have the equivalence

$$
\operatorname{Pic}_{R} \cong \operatorname{Pic}_{\underline{R}}^{\text {loc }}(*)
$$

see Proposition 3.6. We further let $\mathrm{pic}_{R}$ be the connective spectrum such that $\Omega^{\infty} \mathrm{pic}_{R}:=$ $\mathrm{Pic}_{R}$. Proposition 3.6 also shows that we have equivalences resp. an isomorphism

$$
\operatorname{Pic}_{\underline{R}}^{\text {loc }}(M) \cong \operatorname{Pic}_{R}^{M^{t o p}} \cong \Omega^{\infty} \operatorname{Map}\left(\Sigma_{+}^{\infty} M^{t o p}, \operatorname{pic}_{R}\right), \quad \pi_{0}\left(\operatorname{Pic}_{\underline{R}}^{\text {loc }}(M)\right) \cong \operatorname{pic}_{R}^{0}(M),
$$

i.e. $R$-twists are classified by a cohomology group. In particular we get an induced decreasing Atiyah-Hirzebruch filtration

$$
\cdots \subseteq F^{2} \pi_{0}\left(\operatorname{Pic}_{\underline{R}}^{\text {loc }}(M)\right) \subseteq F^{1} \pi_{0}\left(\operatorname{Pic}_{\underline{R}}^{\text {loc }}(M)\right) \subseteq \pi_{0}\left(\operatorname{Pic}_{\underline{R}}^{\text {loc }}(M)\right)
$$

on the abelian group of isomorphism classes of $R$-twists on $M$. 
The cohomology theory of the spectrum of units $\mathrm{gl}_{1}(R)$ of the commutative ring spectrum $R$ is characterized by the natural isomorphism

$$
\mathrm{gl}_{1}(R)^{0}(X) \cong R^{0}(X)^{\times}
$$

for all spaces $X$. We have an equivalence of connective spectra

$$
\operatorname{pic}_{R}\langle 0\rangle \cong \operatorname{bgl}_{1}(R):=\mathrm{gl}_{1}(R)[1] \text {. }
$$

In calculations below we use the following fact:

Lemma 8.4. For $k \geq 0$ the isomorphism

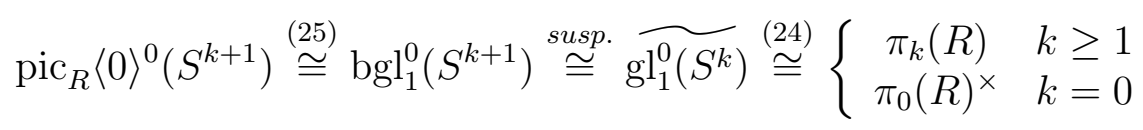

is given by

$$
\operatorname{pic}_{R}\langle 0\rangle^{0}\left(S^{k+1}\right) \ni E \mapsto \nu(E),
$$

where $\nu(E)$ is defined in Definition 3.13 .

Since pic $_{R}$ is a connective spectrum, we have for every connected space $X$ with base point canonical isomorphisms

$$
\operatorname{pic}_{R}^{0}(X) \cong \pi_{0}\left(\operatorname{pic}_{R}\right) \oplus \mathrm{gl}_{1}(R)^{1}(X), \quad \operatorname{pic}_{R}^{-1}(X) \cong \mathrm{gl}_{1}(R)^{0}(X)
$$

By 21) we have identifications

$$
\mathrm{gl}_{1}(R)\langle 0\rangle^{0}(X) \stackrel{\sqrt[24]{\sim}}{\rightarrow} 1+F^{1} R^{0}(X), \quad F^{2} \mathrm{gl}_{1}(R)\langle 1\rangle^{0}(X) \stackrel{\sim}{\rightarrow} 1+F^{2} R^{0}(X)
$$

In general it is quite complicated to understand the spectrum of units $\mathrm{gl}_{1}(R)$ from the structure of $R$. But if $R$ is rational, then one can use a homotopy theoretic version of the exponential map in order to relate $R$ with its units. The details are as follows.

Lemma 8.5. Assume that $Q$ is a commutative algebra in $H \mathbb{Q}$-modules. Then there exists an equivalence of spectra

$$
\exp _{Q}: Q\langle 0\rangle \stackrel{\sim}{\rightarrow} \mathrm{gl}_{1}(Q)\langle 0\rangle
$$

which induces for every space $X$ the exponential map

$$
Q\langle 0\rangle^{0}(X) \rightarrow \operatorname{gl}_{1}(Q)\langle 0\rangle^{0}(X) \quad \beta \mapsto \sum_{n=0}^{\infty} \frac{1}{n !} \beta^{n}
$$

Moreover $\exp _{Q}$ is unique up to homotopy with this property. 
Proof. The spectra $Q\langle 0\rangle, \mathrm{gl}_{1}(Q)$ and $\mathrm{gl}_{1}(Q)\langle 0\rangle$ are $H \mathbb{Q}$-modules. A map between connective $H \mathbb{Q}$-modules is determined up to homotopy by a map of $H$-spaces between the underlying infinite loop spaces (this follows from the Milnor-Moore theorem [MM65]). Furthermore, a map of rational $H$-spaces is determined up to equivalence by the transformation of abelian group-valued functors on the category of finite $C W$-complexes represented by them since there are no phantom maps between rational spectra. Therefore in order to define and characterize the exponential map $\exp _{Q}$ it suffices to check that (28) is a well-defined transformation between abelian group-valued functors on finite $C W$ complexes.

Every element $\beta \in Q\langle 0\rangle^{0}(X) \cong F^{1} Q^{0}(X)$ is nilpotent. Therefore the power series reduces to a finite sum and determines an element

$$
\sum_{n=0}^{\infty} \frac{1}{n !} \beta^{n} \in 1+F^{1} Q^{0}(X) \cong \operatorname{gl}_{1}(Q)\langle 0\rangle^{0}(X) .
$$

Additivity is checked as usual.

Remark 8.6. The exponential map constructed in the lemma is only determined up to homotopy and not up to contractible choices. But we will see in Remark 10.7 that a choice of chain complex modelling the rational spectrum $Q$ allows one to make a prefered choice which is well defined up to contractible choices.

Lemma 8.7. For every manifold $M$ we have a natural isomorphism

$$
\operatorname{Exp}: F^{2} H^{1}(\Omega A(M)) \rightarrow F^{2} \pi_{0}\left(\operatorname{Pic}_{\underline{H A}}^{\text {loc }}(M)\right)
$$

Proof. By Lemma 8.3 the de Rham equivalence induces an isomorphism

$$
F^{2} H^{1}(\Omega A(M)) \stackrel{\sim}{\rightarrow} F^{2} H A^{1}(M) .
$$

By (21) we have an isomorphism

$$
F^{2} H A^{1}(M) \cong H A\langle 0\rangle^{1}(M) .
$$

The exponential map $\exp _{H A}$ induces an isomorphism

$$
\exp _{H A}: H A\langle 0\rangle^{1}(M) \stackrel{\sim}{\rightarrow} \mathrm{gl}_{1}(H A)\langle 0\rangle^{1}(M) .
$$

Finally we have the isomorphism

$$
\begin{aligned}
& \operatorname{gl}_{1}(H A)\langle 0\rangle^{1}(M) \stackrel{\sim}{\longrightarrow} \operatorname{bgl}_{1}(H A)\langle 1\rangle^{0}(M) \underset{[25]}{\sim} \operatorname{pic}_{H A}\langle 1\rangle^{0}(M) \\
& 222 \sqrt{23} \\
& F^{2} \pi_{0}\left(\operatorname{Pic}_{\underline{H A}}^{\text {loc }}(M)\right) \text {. }
\end{aligned}
$$

The isomorphism Exp is defined as the composition of the isomorphisms above. 
In Proposition 6.96 we have constructed a symmetric monoidal transformation

$$
\mathcal{M}: \mathbf{Z}_{A}^{1} \rightarrow \mathrm{Pic}_{\Omega A}^{\text {wloc,fl }}, \quad \omega \mapsto \mathcal{M}(\underline{A}, \omega) .
$$

Its composition with the localization $\mathrm{Pic}_{\Omega A}^{\text {wloc,fl }} \rightarrow \mathrm{Pic}_{\iota(\Omega A)}^{\mathrm{loc}}$ and Eilenberg-MacLane equivalence $\mathrm{Pic}_{\iota(\Omega A)}^{\mathrm{loc}} \stackrel{\sim}{\rightarrow} \mathrm{Pic}_{\underline{H A}}^{\text {loc }}$ yields a symmetric monoidal transformation

$$
H \mathcal{M}: \mathbf{Z}_{A}^{1} \rightarrow \mathrm{Pic}_{\underline{H A}}^{\text {loc }} .
$$

It immediately follows from the definition of $\mathbf{Z}_{A}^{1}$ that $\pi_{0}\left(\mathbf{Z}_{A}^{1}(M)\right) \cong F^{2} H^{1}(\Omega A(M))$.

Proposition 8.8. We assume that $A$ is split. For every manifold $M$ the following diagram commutes

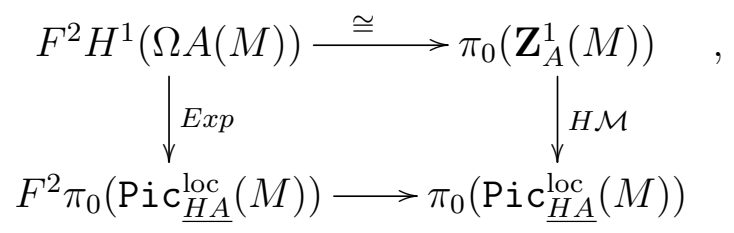

where the lower horizontal map is the inclusion.

The proposition follows from the following two Lemmas.

Lemma 8.9. In order to show Proposition 8.8 it suffices to show that the diagram (29) commutes in the case $M=S^{k}$ for all $k \in \mathbb{N}$.

Proof. Using some natural isomorphisms discussed above we can rewrite the diagram (29) as follows:

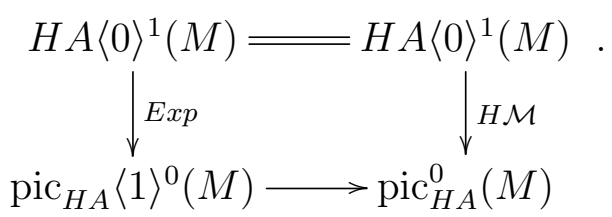

This makes clear that all corners are functors represented by rational $H$-spaces. The horizontal maps represented by maps of $H$-spaces by construction. The transformations of abelian group valued functors Exp and $H \mathcal{M}$ are as well represented by a map between $H$-spaces which are uniquely determined up to homotopy. Finally, the resulting diagram

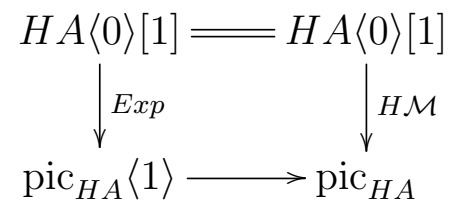

of maps between rational $H$-spaces commutes up to homotopy if and only if it commutes on the level of homotopy groups.

Lemma 8.10. The diagram (29) commutes for $M=S^{k}$ for all $k \in \mathbb{N}$. 
Proof. Note that $H \mathcal{M}$ takes values in the subgroup

$$
F^{1} \pi_{0}\left(\operatorname{Pic}_{\underline{H A}}^{\text {loc }}\left(S^{k+1}\right)\right) \cong \operatorname{pic}_{H A}\langle 0\rangle^{0}\left(S^{k+1}\right) .
$$

By Lemma 8.4 the equivalence class of the element $H \mathcal{M}(\underline{A}, \omega)) \in F^{1} \pi_{0}\left(\operatorname{Pic}_{H A}^{\text {loc }}\left(S^{k+1}\right)\right)$ is completely determined by the class $\nu(H \mathcal{M}(\underline{A}, \omega)) \in \pi_{k}(H A)$ defined in Definition 3.13 .

On the one hand, by Lemma 6.10 we have $\nu(H \mathcal{M}(\underline{A}, \omega))=\sigma([\omega])$. On the other hand, by Lemma 8.4 and the construction of $\operatorname{Exp}$ we have the following equality in $H A^{0}\left(S^{k+1}\right)$ :

$$
1+\sigma^{-1}(\nu(\operatorname{Exp}([\omega])))=\exp ([\omega])=1+[\omega] .
$$

We conclude that $\nu(\operatorname{Exp}([\omega]))=\sigma([\omega])$.

Proposition 8.11. We assume that $A$ is split. Then there exists a canonical equivalence of spectra

$$
\operatorname{gl}_{1}(H A) \cong H\left(H^{0}(A)^{\times}\right) \times H A\langle 0\rangle .
$$

Proof. We consider the fibre sequence

$$
\mathrm{gl}_{1}(H A)\langle 0\rangle \rightarrow \mathrm{gl}_{1}(H A) \rightarrow H\left(H^{0}(A)^{\times}\right) .
$$

Since $A$ is split, we have a natural map of CDGAs

$$
H^{0}(A) \cong Z^{0}(A) \rightarrow A
$$

which induces a split of the fibre sequence $H\left(H^{0}(A)^{\times}\right) \rightarrow \mathrm{gl}_{1}(H A)$. The required equivalence is now constructed using the exponential equivalence Lemma 8.5 :

$$
\mathrm{gl}_{1}(H A) \cong H\left(H^{0}(A)^{\times}\right) \times \mathrm{gl}_{1}(H A)\langle 0\rangle \stackrel{\mathrm{id}^{2} \exp _{H A}^{-1}}{\longrightarrow} H\left(H^{0}(A)^{\times}\right) \times H A\langle 0\rangle .
$$

Using (26), 23), and (21) we get:

Corollary 8.12. We assume that $A$ is split. For every connected manifold $M$ with a fixed base point we have natural isomorphisms

$$
\pi_{0}\left(\operatorname{Pic}_{\underline{H A}}^{\text {loc }}(M)\right) \cong \pi_{0}\left(\operatorname{pic}_{H A}\right) \oplus H\left(H^{0}(A)^{\times}\right)^{1}(M) \oplus F^{2} H A^{1}(M)
$$

and

$$
\pi_{1}\left(\operatorname{Pic}_{\underline{H A}}^{\text {loc }}(M), X\right) \cong H^{0}(A)^{\times} \oplus F^{1} H A^{0}(M)
$$

for every $X \in \operatorname{Pic}_{\underline{H A}}^{\text {loc }}(M)$. 
Corollary 8.13. We assume that $A$ is split. For a connected manifold $M$ with a fixed base point the transformation

$$
\pi_{0}(H \mathcal{M}): \pi_{0}\left(\mathrm{Pic}_{\underline{A}}^{\mathrm{loc}, \mathrm{fl}}(M) \times \mathbf{Z}_{A}^{1}(M)\right) \rightarrow \pi_{0}\left(\operatorname{Pic}_{\underline{H A}}^{\mathrm{loc}}(M)\right)
$$

is given in terms of components of the decompositions obtained in Proposition 6.2 and Corollary 8.12 as the map

$\pi_{0}\left(\mathrm{Pic}_{A}^{\mathrm{ff}}\right) \oplus H\left(Z^{0}(A)^{\times}\right)^{1}(M) \oplus F^{2} H A^{1}(M) \rightarrow \pi_{0}\left(\operatorname{pic}_{H A}\right) \oplus H\left(H^{0}(A)^{\times}\right)^{1}(M) \oplus F^{2} H A^{1}(M)$ with the following description.

1. The first component maps the isomorphism class of $X \in \mathrm{Pic}_{A}^{\mathrm{fl}}$ to the isomorphism class of $H X$ in $\pi_{0}\left(\right.$ pic $\left._{H A}\right)$.

2. Since $A$ is split we have an isomorphism $Z^{0}(A) \cong H^{0}(A)$ which induces the second component

$$
H\left(Z^{0}(A)^{\times}\right)^{1}(M) \cong H\left(H^{0}(A)^{\times}\right)^{1}(M)
$$

3. The third component is the identity by Proposition 8.8 and the construction of the split in Proposition 8.11.

The transformation

$$
\pi_{1}(H \mathcal{M}): \pi_{1}\left(\operatorname{Pic}_{\underline{A}}^{\mathrm{loc}, \mathrm{fl}}(M) \times \mathbf{Z}_{A}^{1}(M)\right) \rightarrow \pi_{1}\left(\operatorname{Pic}_{\underline{H A}}^{\mathrm{loc}}(M)\right)
$$

is given in terms of components

$$
Z^{0}(A)^{\times} \oplus Z^{0}\left(F^{1} \Omega A(M)\right) \rightarrow H^{0}(A)^{\times} \oplus F^{1} H A^{0}(M)
$$

as follows.

1. The first component is the isomorphism $Z^{0}(A)^{\times} \stackrel{\sim}{\rightarrow} H^{0}(A)^{\times}$.

2. The second component maps the cycle $\omega \in Z^{0}\left(F^{1} \Omega A(M)\right)$ to its de Rham cohomology class $[\omega] \in F^{1} H A^{0}(M)$.

In particular, the functor $\mathcal{H} o H \mathcal{M}: \mathcal{H} o \mathbf{Z}_{A}^{1} \rightarrow \mathcal{H} o \mathrm{Pic}_{\underline{H A}}^{\text {loc }}$ is full.

Proof. (of Theorem 8.1): We choose $X \in \mathrm{Pic}_{A}^{\mathrm{fl}}$ such that $H X \cong E(m) \wedge H \mathbb{R}$ as $H A$ modules. Then we have $E(m) \wedge H \mathbb{R}-\underline{H X}(m) \cong 0$ in Pic P $_{A}$. By Corollary 8.13 there

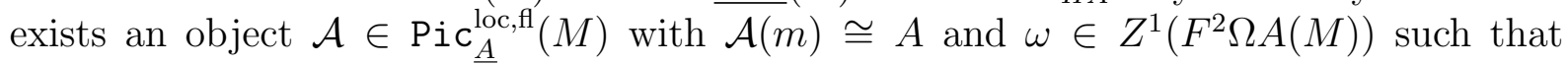
$E \wedge H \mathbb{R} \cong \underline{H X}+\mathcal{M}(\mathcal{A}, \omega) \in \operatorname{Pic}_{\underline{H A}}^{\text {loc }}(M)$. Then there exists an equivalence

$$
d: E \wedge H \mathbb{R} \cong \mathcal{M}\left(\mathcal{A} \otimes_{A} \underline{X}, \omega\right) .
$$


Hence we obtain a differential refinement

$$
\left(E, \mathcal{M}\left(\mathcal{A} \otimes_{A} \underline{X}, \omega\right), d\right) \in \mathcal{T}_{\mathrm{w}_{\hat{R}}}(M)
$$

of $E$.

\section{Part III}

\section{Examples}

\section{Differentially simple spectra}

For a general commutative ring spectrum $R \in \operatorname{CAlg}(\mathcal{S}$ p) it seems to be difficult to single out a good choice of a differential extension $(R, A, c)$. In the present section we introduce the notion of a differentially simple commutative ring spectrum. This class contains all examples which have been considered in applications so far. For a differentially simple commutative ring spectrum we have a very good canonical choice of a differential extension and control of differential twists.

Definition 9.1. We call a commutative ring spectrum $R$ differentially simple, if it has the following properties:

1. $R$ is formal, i.e. there exists an equivalence

$$
c: R \wedge H \mathbb{R} \stackrel{\sim}{\rightarrow} H\left(\pi_{*}(R) \otimes \mathbb{R}\right)
$$

in $\mathrm{CAlg}\left(\operatorname{Mod}_{H \mathbb{R}}\right)$ that induces the identity on homotopy groups.

2. $R$ is rationally connected, i.e. $\pi_{0}(R) \otimes \mathbb{R} \cong \mathbb{R}$.

3. Rationally, the ring spectrum $R$ has no exotic twists, i.e. the canonical assignment

$$
\mathbb{Z} \rightarrow \pi_{0}\left(\mathrm{Pic}_{H A}\right) \quad n \mapsto H A[n]
$$

surjects onto the image of $\pi_{0}\left(\mathrm{Pic}_{R}\right) \rightarrow \pi_{0}\left(\mathrm{Pic}_{H A}\right)$.

In general, the hard piece to check in the above definition is condition (3). However when $R$ is connective, then condition 3. is automatic as follows from the following result.

Proposition 9.2 (Gepner, Antieau [AG12, Theorem 7.9]). If a commutative ring spectrum $E$ is connective and $\pi_{0}(E)$ is a local ring, then $\pi_{0}\left(\mathrm{Pic}_{E}\right)=\mathbb{Z}$.

Corollary 9.3. If $R$ is connective and satisfies condition (1) and (2) in Definition 9.1. then it also satisfies condition (3). 
Proof. If $R$ satisfies condition (1) and (2) in Definition 9.1, then according to Proposition 9.2 the group $\pi_{0}\left(\mathrm{Pic}_{H A}\right)$ consists of the isomorphism classes of the shifts $H A[n]$, and these can be realized by the isomorphism classes of the shifts $A[n]$ in $\pi_{0}\left(\mathrm{Pic}_{A}\right)$.

Recall also that every ring spectrum whose real homotopy is a free graded commutative $\mathbb{R}$-algebra is formal.

Example 9.4. The following spectra are differentially simple:

- ordinary cohomology $H \mathbb{Z}$

- the sphere spectrum $\mathrm{S}$

- connective complex $K$-theory $k u$ and periodic $K$-theory $\mathrm{K}$

- connective real $K$-theory $k o$ and the periodic version $\mathrm{KO}$

- connective topological modular forms tmf and the periodic version TMF

Proof. All examples follow from the last corollary except for the periodic $K$-theories and TMF. But it follows from results of Lennart Mayer and Akhil Mathew MM13 that $\pi_{0}\left(\mathrm{Pic}_{\mathrm{K}}\right)=\mathbb{Z} / 2$ and $\pi_{0}\left(\mathrm{Pic}_{\mathrm{KO}}\right)=\mathbb{Z} / 8$ and $\pi_{0}\left(\mathrm{Pic}_{\mathrm{TMF}}\right)=\mathbb{Z} / 576$. In particular, all classes are represented by shifts.

Theorem 9.5. Assume that $R$ is a differentially simple spectrum and $(R, A, c)$ is the canonical differential extension with $A=\pi_{*}(R) \otimes \mathbb{R}$ and equivalence $c: R \wedge H \mathbb{R} \stackrel{\sim}{\rightarrow} H A$ in $\mathrm{CAlg}\left(\operatorname{Mod}_{H \mathbb{R}}\right)$. Then we have the following assertions:

- Every topological $R$-twist $E$ on a manifold $M$ admits a differential refinement $(E, \mathcal{M}, d)$ which is unique up to (non-canonical) equivalence.

- The sheaf $\mathcal{M}$ of $\Omega A$-modules can be chosen in the form

$$
\mathcal{M}=\mathcal{M}\left(L \otimes_{\mathbb{R}} \underline{A}, w\right)[n]
$$

where $L$ is an invertible locally constant sheaf of $\mathbb{R}$-vector spaces (i.e. the sheaf of parallel sections of a flat real line bundle) and $\omega \in Z^{1}\left(F^{2} \Omega A(M)\right)$.

- The isomorphism class of $L$ and the de Rham class of $\omega$ are uniquely determined by the topological twist E.

Proof. The uniqueness in the first statement follows from Theorem 7.1 and the existence from Theorem 8.1. The second part follows from the fact that $Z^{0}(A)^{\times} \cong \mathbb{R}^{\times}$ 


\section{From differential $b_{1} l_{1}$ to twists}

The main goal of the present section is to construct differential twists from classes in differential $\operatorname{bgl}_{1}(R)$-cohomology. A more precise statement will be given as Theorem 10.1 . after the introduction of necessary notation.

First we review ordinary (non-twisted, non-multiplicative) differential cohomology in the set-up of [BG13], Bun]. Let $p$ be a spectrum, $B$ be a chain complex of real vector spaces and $e: p \wedge H \mathbb{R} \rightarrow H B$ be an equivalence in $\operatorname{Mod}_{H \mathbb{R}}$. The triple $(p, B, e)$ is called a differential data (this is the non-multiplicative version of a differential ring spectrum as introduced in Definition 4.1). For every $n \in \mathbb{Z}$ we define a differential function spectrum $\operatorname{Diff}^{n}(p, B, e) \in \operatorname{Sh}_{\mathcal{S p}}$ as the pull-back

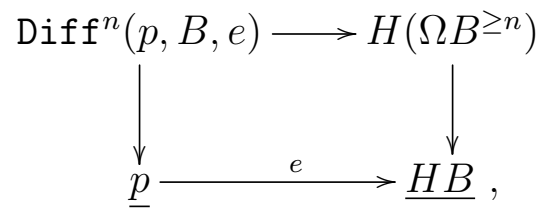

where the right vertical map is induced by the inclusion of chain complexes $\Omega B^{\geq n} \rightarrow \Omega B$ and the de Rham equivalence $H \Omega B \cong \underline{H B}$.

For a smooth manifold $M$ the homotopy groups of the differential function spectrum are given by

$$
\pi_{i}\left(\operatorname{Diff}^{n}(p, B, e)(M)\right) \cong\left\{\begin{array}{cc}
p^{-i}(M) & i<-n \\
p \mathbb{R} / \mathbb{Z}^{-i-1}(M) & i>-n \\
\widehat{p}^{-n}(M) & i=-n
\end{array}\right.
$$

where

$$
\widehat{p}^{n}(M):=\pi_{-n}\left(\operatorname{Diff}^{n}(p, B, e)(M)\right)
$$

is, by definition, the $n$th differential $p$-cohomology of $M$. We refer to [BG13] for further details. For notational reasons we temporarily use the notation $q_{\geq 0}:=q\langle-1\rangle$ for connective covers. For $n \in \mathbb{Z}$ and $k \in \mathbb{N}$ we define

$$
\operatorname{Diff}_{k}^{n}(p, B, e):=\left(\operatorname{Diff}^{n-k}(p, B, e)[n]\right)_{\geq 0} .
$$

Note that $\operatorname{Diff}_{k}^{n}(p, B, e)$ is a sheaf of connective spectra, but not a sheaf of spectra. We now consider a commutative ring spectrum $R$ and a differential refinement $(R, A, c)$. We assume that $A$ is split. In this case we consider the complex

$$
B:=\cdots \rightarrow A^{-2} \rightarrow A^{-1} \rightarrow 0 \rightarrow 0 \rightarrow \ldots
$$

where $A^{-1}$ sits in degree -2 . The complex $B$ will serve as a real model of $\operatorname{bgl}_{1}(R)\langle 1\rangle$. In the following theorem we use the term canonical differential data as in [BG13]. Recall that every spectrum admits canonical differential data. But in general such data is not unique. So the main achievement in the first part of the theorem is contained in the word prefered. 
Theorem 10.1. Let $(R, A, c)$ be a differential data with $A$ split. The spectrum $\operatorname{bgl}_{1}(R)\langle 1\rangle$ admits a prefered canonical differential data

$$
\operatorname{bgl}(R)\langle 1\rangle:=\left(\operatorname{bgl}_{1}(R)\langle 1\rangle, B, e^{-1}\right) .
$$

Furthermore, there exists a canonical map of Picard- $\infty$ stacks

$$
\Omega^{\infty} \operatorname{Diff}_{1}^{0}\left(\operatorname{bgl}_{1}(R)\langle 1\rangle\right) \rightarrow \mathcal{T}_{\mathrm{w}_{\hat{R}}}
$$

The remainder of the present section is devoted to the proof of this theorem. In particular, the map $e$ will be defined in Proposition 10.5. Let us start with more details about the spectra $\operatorname{Diff}_{k}^{n}(p, B, e)$ in the general situation.

Lemma 10.2. 1. The homotopy groups of $\operatorname{Diff}_{k}^{n}(p, B, e)(M)$ are given by

$$
\pi_{i}\left(\operatorname{Diff}_{k}^{n}(p, B, e)(M)\right) \cong\left\{\begin{array}{cc}
0 & i<0 \\
p^{n-i}(M) & 0 \leq i<k \\
\widehat{p}^{n-k}(M) & i=k \\
p \mathbb{R} / \mathbb{Z}^{n-i-1}(M) & i>k
\end{array} .\right.
$$

2. The sheaf of connective spectra $\operatorname{Diff}_{k}^{n}(p, B, e)$ can be written as the pullback in $\mathrm{Sh}_{\mathcal{S}_{\mathrm{p} \geq 0}}$

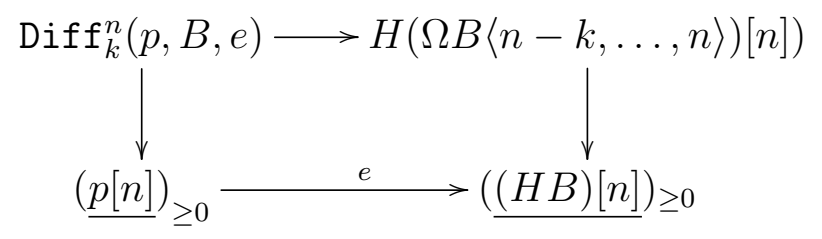

where $\Omega B\langle n-k, \ldots, n\rangle$ is the sheaf in $\mathrm{Sh}_{\mathrm{Ch}} \leq n, \infty$

$$
\Omega B^{n-k} \rightarrow \Omega B^{n-k+1} \rightarrow \ldots \rightarrow \Omega B^{n-1} \rightarrow Z^{n}(\Omega B)
$$

where $Z^{n}(\Omega B)$ sits in degree $n$.

3. There are natural morphisms

$$
\operatorname{Diff}_{0}^{n}(p, B, e) \rightarrow \operatorname{Diff}_{1}^{n}(p, B, e) \rightarrow \operatorname{Diff}_{2}^{n}(p, B, e) \rightarrow \ldots
$$

Proof. 1. The statement follows immediately from the calculation of homotopy groups of $\operatorname{Diff}^{n}(p, B, e)$ as listed in 32 .

2. Note that shifting and taking connective covers both commutes with pullbacks. Thus applying the two operations to the pullback diagram (31) yields (35).

3. This result finally follows from the description of $\operatorname{Diff}_{k}^{n}(p, B, e)$ as a pullback together with the obvious inclusions

$$
\Omega B\langle n, \ldots, n\rangle \rightarrow \Omega B\langle n-1, \ldots, n\rangle \rightarrow \Omega B\langle n-2, \ldots, n\rangle \rightarrow \ldots
$$


Example 10.3. Let $P=H \mathbb{Z}$ be the ordinary Eilenberg-Mac Lane spectrum, $B:=\mathbb{R}[0]$ and $e: H \mathbb{Z} \wedge H \mathbb{R} \rightarrow H B$ be the canonical map. Then the spectrum $\operatorname{Diff}_{k}^{n}(P, B, e)$ has the following interpretation for various values of $n$ and $k$ :

1. $\operatorname{Diff}_{0}^{2}(P, B, e)(M)$ is a 1-type as we see from the homotopy groups. As such it can be identified with the groupoid of line bundles with connection.

2. $\operatorname{Diff}_{1}^{2}(P, B, e)(M)$ is the 1-type, namely the groupid of line bundles without connection.

3. $\operatorname{Diff}_{0}^{3}(P, B, e)(M)$ is a 2-type. It can be identified with the 2-groupoid of gerbes with connection.

4. $\operatorname{Diff}_{1}^{3}(P, B, e)(M)$ is a 2-type which can be identified with the 2-groupoid of gerbes with connection and invertible gerbe bimodules.

5. $\operatorname{Diff}_{2}^{3}(P, B, e)(M)$ is a 2-type which can be identified with the 2-groupoid of gerbes without connection.

We now turn our attention to the case $\operatorname{Diff}_{1}^{0}(p, B, e)$ appearing in the theorem. We give a categorial interpretation of the morphism

$$
H(\Omega B\langle-1, \ldots, 0\rangle) \cong H(\Omega B\langle-1, \ldots, 0\rangle)_{\geq 0} \rightarrow H(\Omega B)_{\geq 0} \cong(\underline{H B})_{\geq 0} .
$$

which is the right vertical map in the diagram 35 that defines $\operatorname{Diff}_{1}^{0}(p, B, e)$. Note that $(\underline{H B})_{\geq 0}$ is a homotopy invariant sheaf of connective spectra. The canonical inclusion of homotopy invariant sheaves into all sheaves fits into an adjunction

$$
i^{h}: \mathrm{Sh}_{\mathcal{S} \mathrm{p}_{\leq 0}}^{h} \leftrightarrows \mathrm{Sh}_{\mathcal{S}_{\mathrm{p} \leq 0}}
$$

and we call $i^{h}$ the homotopification. Given a sheaf $\mathcal{F}$ we shall also refer to the unit $\mathcal{F} \rightarrow i^{h} \mathcal{F}$ as homotopification.

Lemma 10.4. The morphism $H(\Omega B\langle-1, \ldots, 0\rangle) \rightarrow(\underline{H B})_{\geq 0}$ is equivalent to the homotopification.

Proof. Follows immediately from Lemma 7.15 of [BNV13] together with the fact that the Eilenberg-MacLane functor from chain complexes to spectra commutes with homotopification, and the de Rahm equivalence.

We now specialize to the situation of the theorem and set $p:=\operatorname{bgl}_{1}(R)\langle 1\rangle$ and $B$ as in (33). Note that

$$
\Omega B\langle-1, \ldots, 0\rangle \cong F^{1} \Omega A^{0} \rightarrow Z^{1}\left(F^{2} \Omega A\right) .
$$

In Definition 6.8 this two-step complex gave rise to the Picard stack $\mathbf{Z}_{A}^{1}$. By Remark 6.7 we have a canonical equivalence

$$
\Omega^{\infty} H(\Omega B\langle-1, \ldots, 0\rangle) \cong \mathbf{Z}_{A}^{1}
$$


of Picard- $\infty$-stacks.

Recall that we constructed a transformation (restrict 18 to $\{\underline{A}\} \times \mathbf{Z}_{A}^{1}$ )

$$
\mathcal{M}: \mathbf{Z}_{A}^{1} \rightarrow \mathrm{Pic}_{\Omega A}^{\text {wloc,fl }}, \quad \omega \mapsto \mathcal{M}(\underline{A}, \omega) .
$$

of Picard- $\infty$-stacks.

Proposition 10.5. There is a canonical equivalence of spectra

$$
e: H B \rightarrow \operatorname{bgl}_{1}(R)\langle 1\rangle \wedge H \mathbb{R}
$$

and a commutative diagram in $\operatorname{Sh}_{\mathrm{CGrp}(\mathcal{S})}$

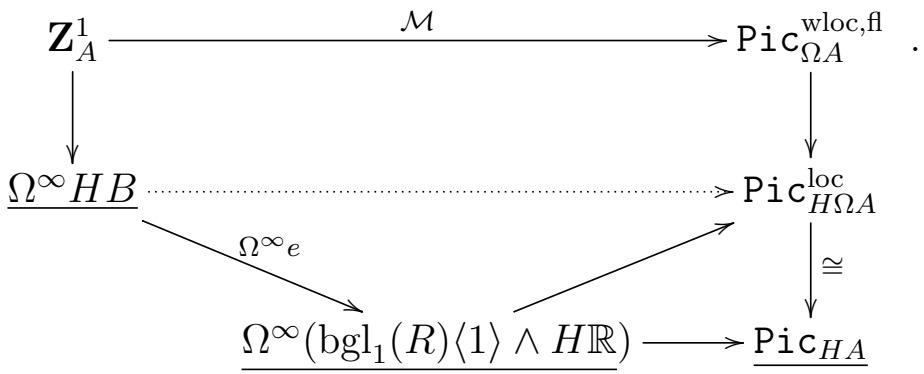

Proof. From Lemma 10.4, the equivalence of categories $\mathrm{Sh}_{\mathcal{S}_{\mathrm{p} \geq 0}} \cong \mathrm{Sh}_{\mathrm{CGrp}(\mathcal{S})}$ and (36) we conclude that the left vertical morphism in diagram (38) is the homotopification. Note that the sheaf $\mathrm{Pic}_{H \Omega A}^{\mathrm{loc}} \cong \mathrm{Pic}_{\underline{H A}}^{\mathrm{loc}}$ in the lower right corner of diagram (38) is homotopy invariant. We get the dotted arrow

$$
\underline{\Omega^{\infty} H B} \rightarrow \mathrm{Pic}_{H \Omega A}^{\mathrm{loc}}
$$

and the upper square from the universal property of the homotopification.

The constant functor

$$
\operatorname{CGrp}(\mathcal{S}) \rightarrow \operatorname{Sh}_{\mathrm{CGrp}}, \quad X \mapsto \underline{X}
$$

is fully faithful. Hence the map

$$
\underline{\Omega^{\infty} H B} \rightarrow \mathrm{Pic}_{H \Omega A}^{\text {loc }} \cong \underline{\mathrm{Pic}_{H A}}
$$

induces the arrow marked by ! in the following diagram in $\operatorname{CGrp}(\mathcal{S})$

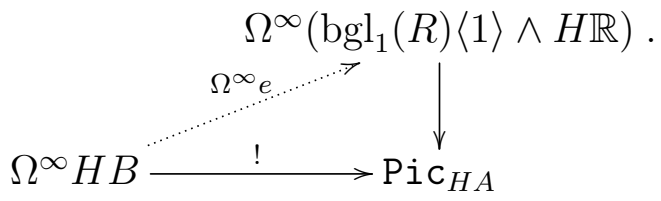

We now observe that

$$
\operatorname{bgl}_{1}(R)\langle 1\rangle \wedge H \mathbb{R} \cong \operatorname{bgl}_{1}(R \wedge H \mathbb{R})\langle 1\rangle \cong \operatorname{bgl}_{1}(H A)\langle 1\rangle \cong \operatorname{pic}_{H A}\langle 1\rangle
$$

and that, under this identification, the right vertical arrow in (39) is just the structure map of a 1-connected covering. Since $\Omega^{\infty} H B$ is one-connected the triangle (39) is unique up to equivalence. It induces corresponding triangle in (38). 
Definition 10.6. Let $(R, A, c)$ be a differential ring spectrum. Then the equivalence (37) determines a prefered canonical differential data

$$
\operatorname{bg} \widehat{1_{1}(R)}\langle 1\rangle:=\left(\operatorname{bgl}_{1}(R)\langle 1\rangle, B, e^{-1}\right)
$$

Remark 10.7. In homotopy the map $e$ induces the map Exp discussed in Proposition 8.8. But note that this proposition only determines the homotopy class of $e$, while here we fix the map itself up to contractible choice. This fact is important to make the domain of the transformation (34) well-defined.

We now construct the asserted canonical map of Picard- $\infty$ stacks

$$
\Omega^{\infty} \operatorname{Diff}_{1}^{0}\left(\operatorname{bgg}_{1}(R)\langle 1\rangle\right) \rightarrow \mathcal{T}_{\mathrm{w}_{\hat{R}}} .
$$

According to Lemma 10.2 the Picard- $\infty$ stack $\Omega^{\infty} \operatorname{Diff}_{1}^{0}\left(\operatorname{bg}_{1}(R)\langle 1\rangle\right)$ is defined as the pullback of the diagram

$$
\underline{\Omega^{\infty} \operatorname{bgl}_{1}(R)\langle 1\rangle} \rightarrow \underline{\Omega^{\infty} H B} \leftarrow \mathbf{Z}_{A}^{1}
$$

Recall that $\mathcal{T}_{\mathrm{w}} \hat{R}$ was defined as the pull-back of Picard- $\infty$-stacks

$$
\mathrm{Pic}_{\underline{R}}^{\text {loc }} \rightarrow \mathrm{Pic}_{H \Omega A}^{\text {loc }} \leftarrow \mathrm{Pic}_{\Omega A}^{\text {wloc,fl }}
$$

Thus it suffices to exhibit a canonical morphism between the two diagrams. To this end we consider the following diagram:

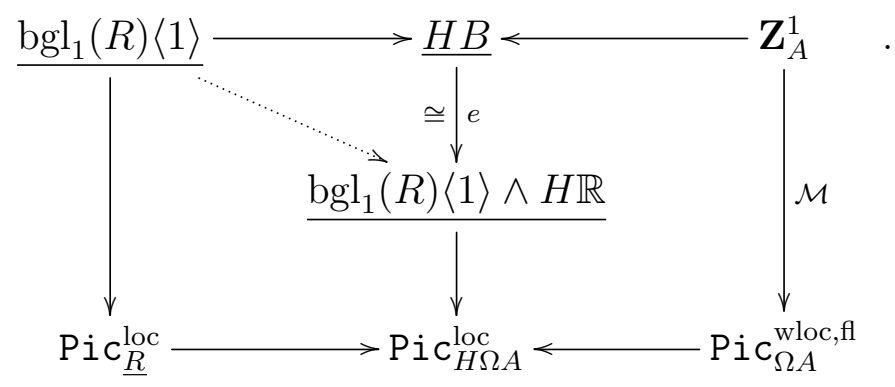

The right-hand part comes from (38). The dotted arrow is the realification map which induces the left upper horizontal map since $e$ is an equivalence. The left lower square commutes since it is equivalent to the sheafification of the realification applied to the one-connective covering map $\operatorname{Pic}_{R}\langle 1\rangle \rightarrow \operatorname{Pic}_{R}$.

This finishes the proof of Theorem 10.1

Corollary 10.8. There is a canonical map of Picard- $\infty$ stacks

$$
\Omega^{\infty} \operatorname{Diff}_{0}^{0}\left(\operatorname{bgg}_{1}(R)\langle 1\rangle\right) \rightarrow \mathcal{T}_{\mathrm{w}_{\hat{R}}}
$$


Proof. Compose the canonical map

$$
\Omega^{\infty} \operatorname{Diff}_{0}^{0}\left(\operatorname{bg} \widehat{\mathrm{l}_{1}(R)}\langle 1\rangle\right) \rightarrow \Omega^{\infty} \operatorname{Diff}_{1}^{0}\left(\operatorname{bg} \widehat{\mathrm{l}_{1}(R)}\langle 1\rangle\right)
$$

from Lemma 10.2 with the canonical map

$$
\Omega^{\infty} \operatorname{Diff}_{1}^{0}\left(\operatorname{bgg}_{1}(R)\langle 1\rangle\right) \rightarrow \mathcal{T}_{\mathrm{w}_{\hat{R}}}
$$

from Theorem 10.1

Remark 10.9. It is a natural question whether one can extend the map 40 to a map from a differential version of $\mathcal{T}_{\mathrm{w}_{R}}$. In fact this can be done, but one has to make some additional choices. We will refrain from doing so here.

\section{Examples of twists}

We fix an integer $n \in \mathbb{N} \backslash\{0\}$ and consider the Picard- $\infty$ groupoid $K(\mathbb{Z}, n)$ (corresponding to the respective Eilenberg MacLane Space). Applying the symmetric monoidal functor $\Sigma_{+}^{\infty}$ from spaces to spectra we obtain a commutative ring spectrum $\Sigma_{+}^{\infty} K(\mathbb{Z}, n)$.

Proposition 11.1. The commutative ring spectrum $\Sigma_{+}^{\infty} K(\mathbb{Z}, n)$ is differentially simple (Definition 9.1). In particular it admits a canonical refinement to a differential spectrum

$$
\left.\Sigma_{+}^{\infty} \widehat{K(\mathbb{Z}}, n\right):=\left(\Sigma_{+}^{\infty} K(\mathbb{Z}, n), \operatorname{Sym}_{\mathbb{R}} \mathbb{R}([n]), \kappa\right)
$$

Proof. We have isomorphisms of rings

$$
\pi_{*}\left(\Sigma_{+}^{\infty} K(\mathbb{Z}, n) \wedge H \mathbb{R}\right) \cong H_{*}\left(\Sigma_{+}^{\infty} K(\mathbb{Z}, n), \mathbb{R}\right) \cong H_{*}(K(\mathbb{Z}, n) ; \mathbb{R}) \cong \operatorname{Sym}_{\mathbb{R}}(\mathbb{R}[n]) .
$$

Since the homotopy of $K(\mathbb{Z}, n) \wedge H \mathbb{R}$ is a polynomial algebra this ring spectrum is formal by Remark 4.3. It also obviously satisfies the remaining conditions for being differentially simple according to Definition 9.1.

From the universal property of the functor $\mathrm{GL}_{1}$ we get a canonical map of Picard- $\infty$ groupoids

$$
K(\mathbb{Z}, n)_{+} \rightarrow \mathrm{GL}_{1}\left(\Sigma_{+}^{\infty} K(\mathbb{Z}, n)\right)
$$

which induces a map of connective spectra

$$
(H \mathbb{Z})[n+1] \rightarrow \operatorname{bgl}_{1}\left(\Sigma_{+}^{\infty} K(\mathbb{Z}, n)\right) .
$$

Since $(H \mathbb{Z})[n+1]$ is one-connected we get a canonical factorization over a map

$$
(H \mathbb{Z})[n+1] \rightarrow \operatorname{bgl}_{1}\left(\Sigma_{+}^{\infty} K(\mathbb{Z}, n)\right)\langle 1\rangle .
$$

From Theorem 10.1 we get a prefered canonical differential data

$$
\operatorname{bgl}_{1}\left(\Sigma_{+}^{\infty K(\mathbb{Z}}, n\right)\langle 1\rangle:=\left(\operatorname{bgl}_{1}\left(\Sigma_{+}^{\infty} K(\mathbb{Z}, n)\langle 1\rangle,\left(\operatorname{Sym}_{\mathbb{R}} \mathbb{R}([n])[1]\right), e^{-1}\right) .\right.
$$

The spectrum $(H \mathbb{Z})[n+1]$ has a canonical differential data

$$
(H \widehat{\mathbb{Z})[n+1]}:=((H \mathbb{Z})[n+1], \mathbb{R}[n+1], c) .
$$


Lemma 11.2. The map of spectra 41) induces a unique equivalence class of maps of canonical differential data

$$
\left(H \overparen{\mathbb{Z})[n+1]} \rightarrow \operatorname{bgl}_{1}\left(\widehat{\sum_{+}^{\infty} K}(\mathbb{Z}, n)\right) .\right.
$$

Proof. By [BG13, Sec. 2.4] there exists such a map whose equivalence class is unique up to the action of

$$
\prod_{k \in \mathbb{Z}} \operatorname{Hom}\left((\mathbb{R}[n+1])^{-k},\left(\operatorname{Sym}_{\mathbb{R}} \mathbb{R}([n])[1]\right)^{-k-1}\right) .
$$

Since this group is trivial the assertion follows.

This map of differential data induces an equivalence class of maps of differential function spectra

$$
\operatorname{Diff}_{1}^{0}((H \widehat{\mathbb{Z})[n}+1]) \rightarrow \operatorname{Diff}_{1}^{0}\left(\operatorname{bgl}_{1}\left(\widehat{\Sigma_{+}^{\infty} K}(\mathbb{Z}, n)\right) .\right.
$$

If we compose this map with the map given in Theorem 10.1, then we get the following maps between Picard- $\infty$-stacks

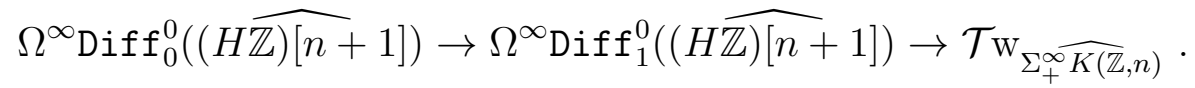

Let $M$ be a smooth manifold. We obtain a commutative diagram of ordinary Picard categories

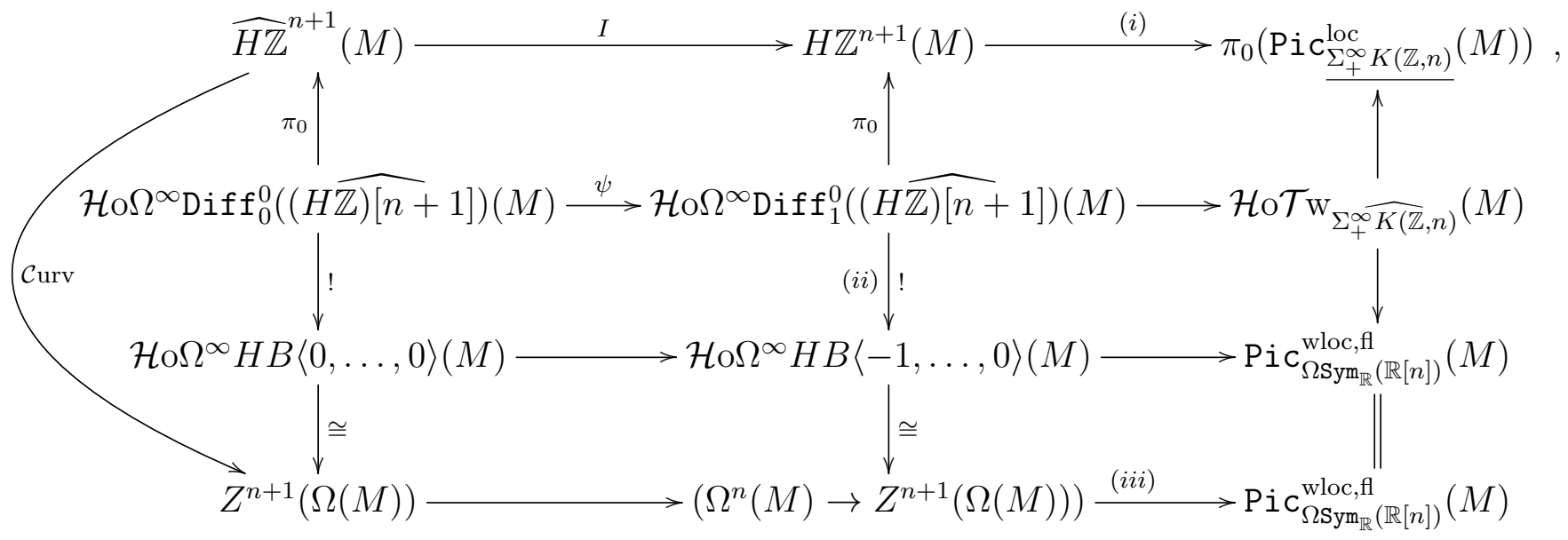

where the arrows marked by ! are induced by the upper horizontal in (35).

Remark 11.3. Let us spell out what this diagram means. Let us call an object

$$
\hat{x} \in \mathcal{H} \mathrm{o} \Omega^{\infty} \operatorname{Diff} f_{0}^{0}((H \widehat{\mathbb{Z})[n+1]})(M)
$$

a differential cycle for differential cohomology class $[\hat{x}] \in \widehat{H \mathbb{Z}}^{n+1}(M)$. Let $\mathcal{C}$ urv $([\hat{x}]) \in$ $Z^{n+1}(\Omega(M))$ and $I([\hat{x}]) \in H \mathbb{Z}^{n+1}(M)$ denote the curvature and the underlying integral cohomology class of $[\hat{x}]$. 
1. We choose an identification $\operatorname{Sym}_{\mathbb{R}}(\mathbb{R}[n]) \cong \mathbb{R}[b]$, where $b \in \operatorname{Sym}_{\mathbb{R}}(\mathbb{R}[n])$ a non-zero element of degree $-n$. The functor (iii) maps a form $\omega \in Z^{n}(\Omega(M))$ to the sheaf

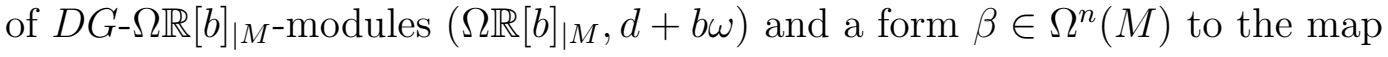

$$
\exp (-\beta b):\left(\Omega \mathbb{R}[b]_{\mid M}, d+b \omega\right) \rightarrow\left(\Omega \mathbb{R}[b]_{\mid M}, d+b \omega^{\prime}\right),
$$

where $\omega^{\prime}:=\omega+d \beta$.

2. The second horizontal line associates to a differential cycle $\hat{x}$ a differential twist which we denote by $(E, \mathcal{M}, d)$.

3. The equivalence class of this differential twist, or what is the same by Theorem 7.1, the equivalence class of the underlying $\Sigma_{+}^{\infty} K(\mathbb{Z}, n)$-twist $E$, is determined by the underlying integral cohomology class $I([\hat{x}])$.

4. The differential cycle $\hat{x}$ determines a closed form $\omega \in \mathcal{C} \operatorname{urv}([\hat{x}]) \in Z^{n+1}(\Omega)$. The lower horizontal line of the diagram maps this form to the sheaf of $D G-\Omega \mathbb{R}[b]_{\mid M^{-}}$ modules

$$
\mathcal{M}:=\left(\Omega \mathbb{R}[b]_{\mid M}, d+b \omega\right)
$$

which is part of the datum of the differential twist $(E, \mathcal{M}, d)$.

5. We now observe that the differential cohomology class $[\hat{x}] \in \widehat{H \mathbb{Z}}^{n+1}(M)$ determines the equivalence class of the $\Sigma_{+}^{\infty} K(\mathbb{Z}, n)$-twist $E$ and the complex $\mathcal{M}$. This additional information contained in the cycle $\hat{x}$ is needed to fix the map $d$.

6. Let us now fix a second differential cycle $\hat{x}^{\prime}$ which gives rise to a differential twist $\left(E^{\prime}, \mathcal{M}^{\prime}, d^{\prime}\right)$, where $\mathcal{M}^{\prime}:=\left(\Omega \mathbb{R}[b]_{\mid M}, d+b \omega^{\prime}\right)$ with $\omega^{\prime}:=\mathcal{C} \operatorname{urv}\left(\left[\hat{x}^{\prime}\right]\right)$. We further consider a morphism $f: \psi(\hat{x}) \rightarrow \psi(\hat{x})^{\prime}$. This morphism induces an equivalence morphism of differential twists $(E, \mathcal{M}, d) \rightarrow\left(E^{\prime}, \mathcal{M}^{\prime}, d^{\prime}\right)$.

7. Using the diagram we can explicitly describe the induced map $\mathcal{M} \rightarrow \mathcal{M}^{\prime}$. The map (ii) associates to the morphism $f$ a form $\beta \in \Omega^{n}(M)$ such that $\omega+d \beta=\omega^{\prime}$. Therefore the morphism $\mathcal{M} \rightarrow \mathcal{M}^{\prime}$ is multiplication by $\exp (-b \beta)$. If the morphism $f$ is in the image of $\psi$, then $\mathcal{M}=\mathcal{M}^{\prime}$ and it acts as the identity.

8. Since $\pi_{1}\left(\operatorname{Diff}_{1}^{0}((\widehat{H \mathbb{Z})[n+1]})(M)) \cong \widehat{H \mathbb{Z}}^{n}(M)\right.$ the second line induces an action of the differential cohomology group $\widehat{H \mathbb{Z}}^{n}(M)$ by automorphisms on differential $\Sigma_{+}^{\infty} K(\mathbb{Z}, b)$-twist. On $\mathcal{M}$ this action is given by multiplication by $\exp (-b \mathcal{C} \operatorname{urv}(y))$.

Proposition 11.4. Let $R$ be a commutative ring spectrum and $\widehat{R}:=(R, A, c)$ be a differential refinement of $R$. A morphism of commutative ring spectra

$$
g: \Sigma_{+}^{\infty} K(\mathbb{Z}, n) \rightarrow R
$$

can be extended to a morphism of differential ring spectra

$$
\left.\widehat{g}: \Sigma_{+}^{\infty} \widehat{K(\mathbb{Z}}, n\right) \rightarrow \widehat{R}
$$

whose equivalence class is unique up to the action of the group $A^{n-1} / \mathrm{im}(d)$. 
Proof. The space of morphism of differential ring spectra from $\left.\Sigma_{+}^{\infty} \widehat{K(\mathbb{Z}}, n\right)$ to $\widehat{R}$ is given by a pull-back

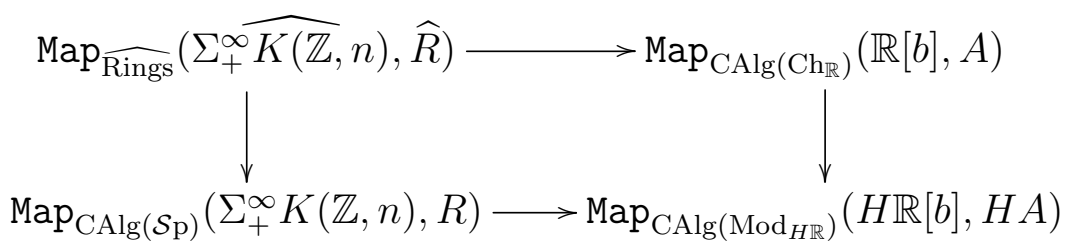

We must calculate the fibre of the left vertical map at $g$ which can be identified with the fibre $\mathbf{X}$ of the right vertical map at the image of $g$. Since $\mathrm{CAlg}\left(\mathrm{Ch}_{\mathbb{R}}\right)$ is a 1-category the right upper corner is discrete. Since $\mathbb{R}[b]$ is freely generated by an element $b$ of cohomological degree $-n$ we get

$$
\pi_{k}\left(\operatorname{Map}_{\mathrm{CAlg}\left(\mathrm{Ch}_{\mathbb{R}}\right)}(\mathbb{R}[b], A)\right) \cong\left\{\begin{array}{cc}
Z^{-n}(A) & k=0 \\
0 & k \geq 1
\end{array}\right.
$$

and

$$
\begin{aligned}
\operatorname{Map}_{\mathrm{CAlg}\left(\operatorname{Mod}_{H \mathbb{R}}\right)}(H \mathbb{R}[b], H A) & \cong \operatorname{Map}_{\mathrm{CAlg}\left(\mathrm{Ch}_{\mathbb{R}, \infty}\right)}(\iota(\mathbb{R}[b]), \iota(A)) \\
& \cong \operatorname{Map}_{\mathrm{Ch}, \infty}(\iota(\mathbb{R}[n]), \iota(A)) \\
& \cong \Omega^{\infty}((H A)[-n])
\end{aligned}
$$

and therefore

$$
\pi_{k}\left(\operatorname{Map}_{\mathrm{CAlg}\left(\operatorname{Mod}_{H \mathbb{R}}\right)}(H \mathbb{R}[b], H A)\right) \cong H^{-n-k}(A) .
$$

We deduce that for $k \geq 1$

$$
\pi_{k}(\mathbf{X}) \cong H^{-n-k}(A)
$$

and $\pi_{0}(X)$ fits into the exact sequence

$$
0 \rightarrow H^{-n-1}(A) \rightarrow \pi_{0}(\mathbf{X}) \rightarrow B^{n}(A) \rightarrow 0
$$

In particular, $\mathbf{X}$ is not empty, and its components are parametrized by $\pi_{0}(X) \cong A^{-n-1} / \mathrm{im}(d)$.

Corollary 11.5. If $A^{-n-1}=0$ in the above setting, then the extension $\widehat{g}$ is unique up to non-canonical equivalence.

Assume that we have a morphism of commutative ring spectra $g: \Sigma_{+}^{\infty} K(\mathbb{Z}, n) \rightarrow R$ and a differential refinement $(R, A, c)$ with $A^{-1-n}=0$. Then by composing the morphism

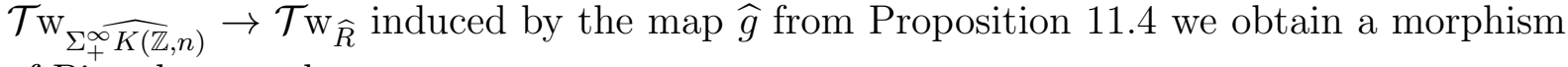
of Picard- $\infty$-stacks

$$
\underline{\mathbb{Z}} \times \Omega^{\infty} \operatorname{Diff}_{1}^{0}(H \widehat{\mathbb{Z}[n+1]}) \rightarrow \mathcal{T}_{\mathrm{w}_{\widehat{R}}}(M)
$$


where the additional $\mathbb{Z}$-factor introduces degree shifts. Restricting the grading of the twisted function spectrum (Definition 4.11) to $\mathbb{Z} \times \Omega^{\infty} \operatorname{Diff}_{1}^{0}(H \widehat{\mathbb{Z}[n+1]})$ thus yields a sheaf of graded ring spectra

$$
M \mapsto\left(\underline{\mathbb{Z}} \times \Omega^{\infty} \operatorname{Diff}_{1}^{0}(H \widehat{\mathbb{Z}[n+1]})(M), \operatorname{Diff}(\ldots)(M)\right)
$$

and a functor

$$
\mathcal{M f} \mathrm{f}^{o p} \rightarrow \operatorname{GrRing}, \quad M \mapsto\left(\mathbb{Z} \times \mathcal{H} \circ \Omega^{\infty} \operatorname{Diff}_{1}^{0}(H \widehat{\mathbb{Z}[n+1]})(M), \widehat{R} \cdots(M)\right) \overleftrightarrow{3}^{3}
$$

Remark 11.6. Using Remark 11.3 (and the notation introduced there) we obtain the following.

1. For every differential cycle $\hat{x} \in \Omega^{\infty} \operatorname{Diff}_{0}^{0}(H \widehat{\mathbb{Z}[n+1]})(M)$ and integer $k \in \mathbb{Z}$ we obtain an abelian group $\widehat{R}^{k+\psi(\hat{x})}$.

2. The curvature map for this group takes values in $Z^{k}(\Omega A, d+\omega b)$, where $\omega=$ $\mathcal{C} \operatorname{urv}([\hat{x}])$, and we use that $A$ is an $\mathbb{R}[b]$-module.

3. For every morphism $f: \psi(\hat{x}) \rightarrow \psi\left(\hat{x}^{\prime}\right)$ we get an isomorphism $\psi(f): \widehat{R}^{k+\phi(\hat{x})} \rightarrow$ $\widehat{R}^{k+\phi\left(\hat{x}^{\prime}\right)}$ and a form $\beta \in \Omega^{n-1}$ such that $d \beta+\omega=\omega^{\prime}$, and

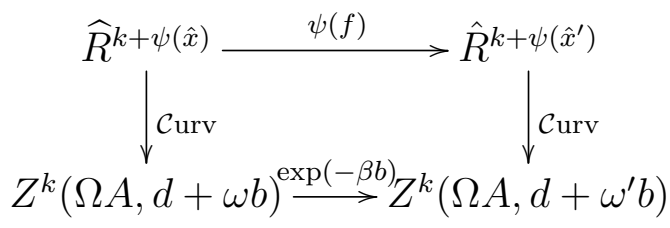

commutes.

4. For a pair of differential cycles $\hat{x}, \hat{x}^{\prime}$ and integers $k, k^{\prime} \in \mathbb{Z}$ we have a diagram

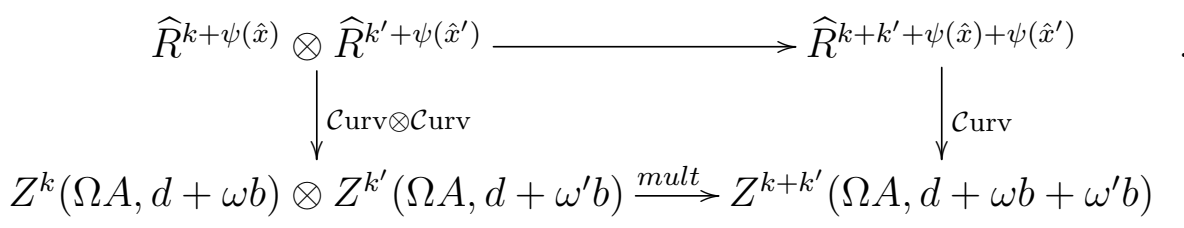

Example 11.7. A trivial example where the above applies is $R:=\Sigma_{+}^{\infty} K(\mathbb{Z}, n)$ and $g$ the identity. But the classical example is complex $K$-theory. It is known by [AGG11] that there is an essentially unique equivalence class of maps of Picard- $\infty$-groupoids $K(\mathbb{Z}, 2) \rightarrow$ $\mathrm{GL}_{1}(\mathrm{~K})$ (see also 1. in Example 11.8). We therefore get a canonical map $\Sigma_{+}^{\infty} K(\mathbb{Z}, 2) \rightarrow \mathrm{K}$ of commutative ring spectra. Since K is even, Corollary 11.5 applies. We therefore get a differential twisted $K$-theory $\widehat{\mathbf{K U}}^{\cdots}(M)$ which is graded by $\mathbb{Z} \times \Omega^{\infty} \operatorname{Diff}_{1}^{0}(\widehat{H \mathbb{Z}[3]})(M)$. The structures spelled out in Remark 11.6 have partially been constructed using explicit models in CMW09, GT10]. In particular, the real approximation is given by the usual periodic de Rham complex $\mathcal{M}\left(\underline{\mathbb{R}\left[b, b^{-1}\right]}, \omega b\right)$, where $\omega=\mathcal{C} \operatorname{urv}([\hat{x}]) \in Z^{3}(\Omega)$ and $\operatorname{deg}(b)=-2$. 
Example 11.8. Other examples of interesting maps of ring spectra $\Sigma_{+}^{\infty} K(\mathbb{Z}, n) \rightarrow R$ can be constructed following ideas of Ando, Blumberg and Gepner [ABG10]. We consider the Picard- $\infty$-groupoid $B O:=\Omega^{\infty} \mathbf{K O}\langle 0\rangle$. Assume that we are given a sequence of maps between Picard- $\infty$-groupoids

$$
K(\mathbb{Z}, n) \rightarrow X \rightarrow B O
$$

such that the composition is nullhomotopic. The Thom spectrum construction

$$
M: \operatorname{CGrp}(\mathcal{S}) / B O \rightarrow \operatorname{CAlg}(\mathcal{S p}),(X \rightarrow B O) \mapsto M X
$$

functorially associates to every Picard- $\infty$-groupoid over $B O$ a commutative ring spectrum. Since we assume that $K(\mathbb{Z}, n) \rightarrow B O$ is null-homotopic we have an equivalence $M K(\mathbb{Z}, n) \cong \Sigma_{+}^{\infty} K(\mathbb{Z}, n)$. Hence the sequence 42 induces a map

$$
\Sigma_{+}^{\infty} K(\mathbb{Z}, n) \rightarrow M X
$$

In the following we discuss examples of sequences $(42)$ from maps between classical groups.

1. The central extension

$$
1 \rightarrow U(1) \rightarrow \operatorname{Spin}^{c} \rightarrow S O \rightarrow 1
$$

together with the identification $B U(1) \cong K(\mathbb{Z}, 2)$ and the map $B S O \rightarrow B O$ induces the sequence

$$
K(\mathbb{Z}, 2) \rightarrow B \text { Spin }^{c} \rightarrow B O .
$$

The construction produces a morphism of commutative ring spectra

$$
\Sigma_{+}^{\infty} K(\mathbb{Z}, 2) \rightarrow M \text { Spin }^{c}
$$

Note that $\pi_{3}\left(M \operatorname{Spin}^{c}\right) \otimes \mathbb{Q}=0$ so that we can apply Corollary 11.5 . If we compose this morphism with the Atiyah-Bott-Shapiro orientation $A: M \operatorname{Spin}^{c} \rightarrow \mathrm{K}$, then we get the map

$$
\Sigma_{+}^{\infty} K(\mathbb{Z}, 2) \rightarrow \mathrm{K}
$$

discussed in Example 11.7 .

2. We have a fibre sequence

$$
K(\mathbb{Z}, 3) \rightarrow \text { BString } \rightarrow \text { BSpin }
$$

It provides a morphism of commutative ring spectra

$$
\Sigma_{+}^{\infty} K(\mathbb{Z}, 3) \rightarrow \text { MString }
$$

If we compose this with the string orientation MString $\rightarrow t m f$ of the spectrum of topological modular forms $\operatorname{tm} f$ (constructed by Ando, Hopkins and Rezk) we get a map

$$
\Sigma_{+}^{\infty} K(\mathbb{Z}, 3) \rightarrow t m f
$$


of commutative ring spectra.

Since $\pi_{4}(M$ String $) \otimes \mathbb{Q}=0$ and $\pi_{4}(t m f) \otimes \mathbb{Q}=0$ we can apply Corollary 11.5 to both cases.

Further note that $\pi_{3}(M$ String $) \otimes \mathbb{Q}=0$ and $\pi_{3}(t m f) \otimes \mathbb{Q}=0$. Therefore in both cases for every differential 4-cycle the curvature of the corresponding twisted MString or tmf-cohomology takes values in the cycles of the untwisted de Rham complexes $\left(\Omega\left(\pi_{*}(M\right.\right.$ String $\left.\left.) \otimes \mathbb{R}\right), d\right)$ or $\left(\Omega\left(\pi_{*}(t m f) \otimes \mathbb{R}\right), d\right)$, and morphisms between differential cycles act by the identity on the form level.

\section{The twisted Atiyah-Hirzebruch spectral sequence}

We consider a commutative ring spectrum $R$ and a differential refinement $\widehat{R}=(R, A, c)$. Assume that we are given an $R$-twist $E \in \mathrm{Pic}_{R}^{\text {loc }}(M)$ which is trivial on the 1-skeleton of $M$, i.e. $E$ is classified by a map $M \rightarrow \mathrm{BGL}_{1}(R)\langle 1\rangle$. We have shown in Section 8 that there exists a twisted de Rham complex $\mathcal{M}=\mathcal{M}(\underline{A}, \omega)$ with $\omega \in Z^{1}\left(F^{2} \Omega A(M)\right)$ such that $E$ refines to a differential twist $(E, \mathcal{M}, d)$. In this section we demonstrate how the cohomology class of $\omega$ can effectively be determined by the differentials in the Atiyah-Hirzebruch spectral sequence associated to the twist $E$.

More generally, consider $\mathcal{A} \in \operatorname{Pic}_{A}^{\text {loc,fl }}(M)$ and the sheaf $\mathcal{M}(\mathcal{A}, \omega)$ of $D G-\Omega A_{\mid M}$-modules introduced in Definition 6.4. It has a decreasing filtration

$$
F^{p} \mathcal{M}(\mathcal{A}, \omega):=\Omega_{\mid M}^{\geq p} \otimes_{\mathbb{R}} \mathcal{A}
$$

which turns it in into a sheaf of filtered $D G$-modules over the similarly filtered sheaf of $D G$-algebras $\Omega A_{\mid M}$. This filtration induces a filtration of the complex of global sections $\mathcal{M}(\mathcal{A}, \omega)(M)$ and therefore a spectral sequence $\left(\mathcal{M}(\mathcal{A}, \omega) E_{r}, d_{r}\right)$ which converges to $H^{*}(\mathcal{M}(\mathcal{A}, \omega)(M))$. The spectral sequence has the structure of a module over the corresponding spectral sequence $\left(\Omega A E_{r}, d_{r}\right)$ for $\Omega A_{\mid M}$.

We let $\operatorname{sing}(M)$ denote the smooth singular complex of $M$ and

$$
|\operatorname{sing}(M)|:=\bigcup_{n \in \mathbb{N}} \operatorname{sing}(M)[n] \times \Delta^{n} / \sim
$$

be its geometric realization. We have a canonical evaluation map $\phi:|\operatorname{sing}(M)| \rightarrow M$ such that $\phi(\sigma, t)=\sigma(t)$, where $\sigma: \Delta^{n} \rightarrow M$ is a point in $\operatorname{sing}(M)[n]$ and $t \in \Delta^{n}$. We consider $|\operatorname{sing}(M)|$ as a piecewise smooth manifold on which we can consider differential forms and sheaves of spectra. The map $\phi$ is piecewise smooth.

The piecewise smooth manifold $|\operatorname{sing}(M)|$ has an increasing filtration

$$
\emptyset=\operatorname{sing}(M)_{-1} \subseteq|\operatorname{sing}(M)|_{0} \subseteq|\operatorname{sing}(M)|_{1} \subseteq \ldots
$$


by skeleta.

Let $X \in \operatorname{Sh}_{\mathcal{S}}(M)$. Then we can consider the pull-back $\phi^{*} X \in \operatorname{Sh}_{\mathcal{S p}}(|\operatorname{sing}(M)|)^{4}$. The filtration of $|\operatorname{sing}(M)|$ induces, by definition, the Atiyah-Hirzebruch spectral sequence $\left({ }_{X} E_{r}, d_{r}\right)$ which converges to $\pi_{*}(X(M))$.

Lemma 12.1. We have a canonical isomorphism of spectral sequences $\left(\mathcal{M}(\mathcal{A}, \omega) E_{r}, d_{r}\right)$ and $\left(H \mathcal{M}(\mathcal{A}, \omega) E_{r}, d_{r}\right)$ for $r \geq 2$.

Proof. The filtration of $|\operatorname{sing}(M)|$ induces a decreasing filtration of the complex

$\cdots \subseteq \tilde{F}^{1} \mathcal{M}\left(\phi^{*} \mathcal{A}, \phi^{*} \omega\right)(|\operatorname{sing}(M)|) \subseteq \tilde{F}^{0} \mathcal{M}\left(\phi^{*} \mathcal{A}, \phi^{*} \omega\right)(|\operatorname{sing}(M)|)=\mathcal{M}\left(\phi^{*} \mathcal{A}, \phi^{*} \omega\right)(|\operatorname{sing}(M)|)$,

where $\tilde{F}^{k} \mathcal{M}\left(\phi^{*} \mathcal{A}, \phi^{*} \omega\right)(|\operatorname{sing}(M)|)$ is the kernel of the restriction to $|\operatorname{sing}(M)|_{k-1}$. We let $\left(\tilde{E}_{r}, \tilde{d}_{r}\right)$ be the associated spectral sequence.

The piecewise smooth map $\phi$ induces a morphism of complexes

$$
\phi^{*}: \mathcal{M}(\mathcal{A}, \omega)(M) \rightarrow \mathcal{M}\left(\phi^{*} \mathcal{A}, \phi^{*} \omega\right)(|\operatorname{sing}(M)|)
$$

and satisfies

$$
\phi^{*} F^{p} \mathcal{M}(\mathcal{A}, \omega)(M) \subseteq \tilde{F}^{p}\left(\mathcal{M}\left(\phi^{*} \mathcal{A}, \phi^{*} \omega\right)(|\operatorname{sing}(M)|)\right)
$$

We therefore get a map of spectral sequences $\left(\mathcal{M}(\mathcal{A}, \omega) E_{r}, d_{r}\right) \rightarrow\left(\tilde{E}_{r}, \tilde{d}_{r}\right)$.

We check that it is an isomorphism on the second page and therefore on all higher pages. To this end we calculate calculate $\tilde{E}_{2}$. We have

$$
\tilde{E}_{0}^{p, q} \cong F^{p} \mathcal{M}\left(\phi^{*} \mathcal{A}, \phi^{*} \omega\right)^{q+p}(|\operatorname{sing}(M)|) / F^{p-1} \mathcal{M}\left(\phi^{*} \mathcal{A}, \phi^{*} \omega\right)^{q+p}(|\operatorname{sing}(M)|) .
$$

This complex calculates the relative sheaf cohomology

$$
H^{q+p}\left(\operatorname{sing}(M)_{p}, \operatorname{sing}(M)_{p-1} ; \mathcal{M}\left(\phi^{*} \mathcal{A}, \phi^{*} \omega\right)\right)
$$

This cohomology group is a product over the cohomology of all $p$-simplices in $|\operatorname{sing}(M)|$ relative to their boundaries with coefficients in the restrictions of $\mathcal{M}\left(\phi^{*} \mathcal{A}, \phi^{*} \omega\right)$. Since the data $\phi^{*} \mathcal{A}$ and $\phi^{*} \omega$ can be trivialized on the simplicies, each $p$-simplex contributes a factor of $H^{q}(A)$. Using the fact that $\omega$ belongs to $F^{2}(\Omega A(M))$ we now observe that $\left(\tilde{E}_{1}^{p, q}, \tilde{d}_{1}\right) \cong\left(C^{p}\left(\operatorname{sing}(M), H^{q}(\mathcal{A})\right), d_{1}\right)$ is exactly the reduced singular complex associated to the local system $H^{*}(\mathcal{A})$. Hence we get

$$
\tilde{E}_{2}^{p, q} \cong H^{p}\left(\operatorname{sing}(M) ; H^{q}(\mathcal{A})\right)
$$

\footnotetext{
${ }^{4}$ This is actually an abuse of notation since $|\operatorname{sing}(M)|$ is not an object of $\mathcal{M}$ f. Nevertheless we think that is is clear from the context what is ment here.
} 
We now consider the spectral sequence $\left(\mathcal{M}(\mathcal{A}, \omega) E_{r}, d_{r}\right)$ in greater detail. We have

$$
\mathcal{M}(\mathcal{A}, \omega) E_{0}^{p, q} \cong\left(\Omega^{p} \otimes \mathcal{A}^{q}\right)(M), \quad d_{0}=1 \otimes d_{\mathcal{A}} .
$$

We get

$$
\mathcal{M}(\mathcal{A}, \omega) E_{1}^{p, q} \cong\left(\Omega^{p} \otimes H^{q}(\mathcal{A})\right)(M), \quad d_{1}=d_{\Omega} \otimes 1
$$

It follows that

$$
\mathcal{M}(\mathcal{A}, \omega) E_{2}^{p, q} \cong H^{p}\left(M ; H^{q}(\mathcal{A})\right)
$$

Since the Eilenberg-MacLane equivalence is an equivalence between sheaves of complexes and sheaves of $H \mathbb{Z}$-modules the descent spectral sequences for $\left(H \mathcal{M}(\mathcal{A}, \omega) E_{r}, d_{r}\right)$ and $\left(\tilde{E}_{r}, \tilde{d}_{r}\right)$ associated to the skeletal filtration of $|\operatorname{sing}(M)|$ are isomorphic.

This finally implies the assertion.

Lemma 12.2. The spectral sequence $\left({ }_{\Omega A} E_{r}, d_{r}\right)$ degenerates at the second page.

Proof. If $X \cong \underline{Q}_{\mid M}$ is the constant sheaf generated by a spectrum $Q \in \mathcal{S}$ p, then $\left({ }_{X} E_{r}, d_{r}\right)$ is the classical Atiyah-Hirzebruch spectral sequence. It is a well-known fact, that it degenerates at the second page if $Q$ is a rational spectrum. Since $\Omega A \cong \underline{H A}$ and $H A$ is rational we conclude that $\left({ }_{\Omega A} E_{r}, d_{r}\right) \cong\left(\underline{{ }_{H A}} E_{r}, d_{r}\right)$ degenerates at the second page.

We write $\omega=\sum_{n \geq 2} \omega^{n}$, where $\omega^{n} \in\left(\Omega^{n} \otimes \underline{A}^{1-n}\right)(M)$. Let $r \in \mathbb{N}$ be such that $\omega^{n}=0$ for all $n<r$. Then we have $\left(1 \otimes d_{A}\right) \omega^{r}=0$ and $\left(d_{\Omega} \otimes 1\right) \omega^{r}=(-1)^{r}\left(1 \otimes d_{A}\right) \omega^{r+1}$. We see that $\omega^{r}$ represents a class $\left[\omega^{r}\right] \in H^{r}\left(M ; \underline{\left.H^{1-r}(A)\right)} \cong{ }_{\Omega A} E_{2}^{r, 1-r}\right.$.

In the following Lemma we write $d(\omega)_{r}$ for the differential of $\mathcal{M}(\underline{A}, \omega) E_{r}^{p, q}$, and $d_{r}$ for the differential of $\Omega_{\Omega A} E_{r}^{p, q}$.

Lemma 12.3. For $2 \leq i \leq r$ we have

$$
\mathcal{M}(\underline{A}, \omega) E_{i}^{p, q} \cong{ }_{\Omega A} E_{i}^{p, q},
$$

and $d(\omega)_{i}=0$ for all $2 \leq i<r$. Furthermore, $d(\omega)_{r}=d_{r}+\left[\omega^{r}\right]$.

Proof. This is a consequence of the fact that we have a canonical identification of the filtered $\mathbb{Z}$-graded vector spaces $\mathcal{M}(\underline{A}, \omega)(M)^{\sharp} \cong \Omega A(M)^{\sharp}$ and the difference of the differentials $d(\omega)-d=\omega$ increases the filtration by $r$ steps.

Lemma 12.4. The following assertions are equivalent:

1. We have an isomorphism $\mathcal{M}(\underline{A}, \omega) \cong \mathcal{M}(\underline{A}, 0)$ of $D G$-module sheaves over $\Omega A$.

2. The class $[\omega] \in H^{1}(\Omega A(M))$ vanishes. 
3. The spectral sequence $\left(\mathcal{M}(\underline{A}, \omega) E_{r}, d(\omega)_{r}\right)$ degenerates at the second page.

Proof. We show 2. $\Rightarrow 1 . \Rightarrow 3 . \Rightarrow 2$.

If $[\omega]=0$, then $\mathcal{M}(\underline{A}, \omega) \cong \mathcal{M}(\underline{A}, 0)$ by 16$)$.

We now assume that $\mathcal{M}(\underline{A}, \omega) \cong \mathcal{M}(\underline{A}, 0)$. In this case, as a consequence of the Lemmas 12.1 and 12.2 , the spectral sequence $\left(\mathcal{M}(\underline{A}, \omega) E_{r}, d(\omega)_{r}\right)$ degenerates at the second page.

We now assume that the spectral sequence $\left(\mathcal{M}(\underline{A}, \omega) E_{r}, d(\omega)_{r}\right)$ degenerates at the second page. We argue by contradiction and assume that $[\omega] \neq 0$. Then there exists a maximal $r \geq 2$ with $[\omega] \in F^{r} H^{1}(M, A)$. We can assume that $\omega \in Z^{1}\left(F^{r} \Omega A(M)\right)$ and $\left[\omega^{r}\right] \in H^{r}\left(M, H^{1-r}(A)\right)={ }_{\Omega A} E_{2}^{r, 1-r}$ does not vanish. The unit $1 \in A$ induces a unit $1 \otimes 1 \in \Omega^{0} \otimes \underline{A}$ with image $1 \in H^{0}(\Omega A(M))$. This unit is detected by a unit $1_{r} \in E_{r}^{0,0}$. By Lemma 12.3 we have $0=d(\omega)_{r}\left(1_{r}\right)=\left[\omega^{r}\right]$. This is a contradiction.

We can now describe an algorithm which allows to determine the real approximation $\mathcal{M}=\mathcal{M}(\underline{A}, \omega)$ of a given $R$-twist $E \in \operatorname{Pic}_{R}^{\text {loc }}(M)$. The main point is to determine the cohomology class of $\omega$ such that

$$
(E \wedge H \mathbb{R}) \wedge_{H \Omega A} H \mathcal{M}(\underline{A},-\omega) \cong H \Omega A
$$

Since we assume that $E$ is trivialized on the one-skeleton of $M$ we have an isomorphism

$$
(E \wedge H \mathbb{R}) \wedge_{H \Omega A} H \mathcal{M}(\underline{A},-\omega) E_{2}^{p, q} \cong H^{p}\left(M, \underline{H^{q}(A)}\right) \cong{ }_{\Omega A} E_{2}^{p, q}
$$

We write $\tilde{d}_{r}$ for the differential of the spectral sequence on the left-hand side.

Assume that we have found $\omega \in Z^{1}\left(F^{2} \Omega A(M)\right)$ such that this isomorphism extends to an isomorphism of the pages $2, \ldots, r$. Then we consider the difference $\left(\tilde{d}_{r}-d_{r}\right)\left(1_{r}\right)=: x \in$ ${ }_{\Omega A} E_{2}^{r, 1-r}$. It follows from the module structure over ${ }_{\Omega A} E_{r}$ that then $\tilde{d}_{r}-d_{r}=x$. There exists $\kappa \in Z^{1}\left(F^{r} \Omega A(M)\right)$ such that the cohomology class of $\kappa$ is detected by $x$. After replacing $\omega$ by $\omega+\kappa$ we can assume that $x=0$ and our isomorphism of pages persists to the $(r+1)$ th page.

We now proceed by induction on $r$. Since $M$ is finite-dimensional, after finitely many iterations we have found a form $\omega \in Z^{1}\left(F^{2} \Omega A(M)\right)$ such that the spectral sequence for $(E \wedge H \mathbb{R}) \wedge_{H \Omega A} H \mathcal{M}(\underline{A},-\omega)$ degenerates at the second term. It now follows from Lemma 12.4 that $E \wedge H \mathbb{R} \cong H \mathcal{M}(\underline{A}, \omega)$.

Example 12.5. We illustrate this procedure in the classical case of twisted complex $K$ theory which has already been considered in Example 11.7. In this case the procedure terminates after the first iteration and reproduces a well-known fact. 
The map of Picard- $\infty$ groupoids $K(\mathbb{Z}, 2) \rightarrow \mathrm{GL}_{1}(\mathrm{~K})$ induces a map of spaces $K(\mathbb{Z}, 3) \rightarrow$ $\mathrm{BGL}_{1}(\mathrm{~K})$ by one-fold delooping. This map classifies a twist $E \in \operatorname{Pic}_{K}^{\text {loc }}(K(\mathbb{Z}, 3))$.

The Atiyah-Hirzebruch spectral sequence $\left({ }_{E} E_{r}, d_{r}\right)$ has been investigated in AS06, Sec. 4]. Let $\eta \in H^{3}(K(\mathbb{Z}, 3) ; \mathbb{Z})$ denote the canonical class. It induces a class

$$
\eta b \in H^{1}\left(K(\mathbb{Z}, 3) ; \mathbb{R}\left[b, b^{-1}\right]\right) .
$$

The calculation of Atiyah-Segal shows that ${ }_{E \wedge H \mathbb{R}} E_{3}^{p, q} \cong H^{p}\left(K(\mathbb{Z}, 3) ; \mathbb{R}\left[b, b^{-1}\right]^{q}\right)$ and $d_{3}=$ $\eta b$. Note that rationally $K(\mathbb{Z}, 3)$ behaves like $S^{3}$. One checks that that ${ }_{E \wedge H \mathbb{R}} E_{4}=0$.

Let now $f: M \rightarrow K(\mathbb{Z}, 3)$ be a map from a smooth manifold representing a class $x \in$ $H^{3}(M ; \mathbb{Z})$. Then, if we take a form $\omega \in Z^{3}(\Omega(M))$ such that $[\omega]=x \otimes \mathbb{R}$, there exists an equivalence

$$
f^{*} E \wedge H \mathbb{R} \cong H \mathcal{M}\left(\underline{\mathbb{R}\left[b, b^{-1}\right]}, \omega b\right) .
$$

Thus our procedure reproduces the complex found in Example 11.7.

\section{Part IV}

\section{Appendix}

\section{A Technical facts about graded ring spectra}

Here we will prove some technical facts about graded ring spectra (defined in Section 2). More precisely we show that the constructions that are required to define twisted and differential twisted cohomology admit good functorial properties.

Let $\left(\mathcal{C a t}_{\infty}\right)_{\star}$ denote the total space of the universal cocartesian fibration over $\mathcal{C}_{\infty} t_{\infty}$. See Lur09, Section 3.3.2] for more details. Roughly speaking objects of $\left(\mathcal{C} \mathrm{at}_{\infty}\right)_{\star}$ are given by $\infty$-categories with a chosen base point. However the morphisms in $\left(\mathcal{C a t}_{\infty}\right)_{\star}$ are not required to preserve this object up to isomorphism, but only up to a possible non-invertible morphism. Thus the overcategory $\left({\mathcal{C} a t_{\infty}}_{* /}\right.$ is a subcategory of $\left(\mathcal{C} \text { at }{ }_{\infty}\right)_{\star}$ with the same objects but less morphisms. We will also refer to the category $\left(\mathcal{C} a t_{\infty}\right)_{\star}$ as the lax slice of $\mathcal{C}_{\infty}{ }_{\infty}$ under the point.

Remark A.1. Note that $\mathcal{C}_{\infty}$ and $\left(\mathcal{C} t_{\infty}\right)_{\star}$ are neither small nor large. The reason is that $\mathcal{C}$ at ${ }_{\infty}$ contains all large categories. Thus we need to work with a third universe, which we call very large. Then $\mathcal{C}_{a} t_{\infty}$ is very large. But the fibres of the universal fibration are large. Thus $\mathcal{C}_{\infty}$ classifies cocartesian fibrations $X \rightarrow S$ between $\infty$-categories $X$ and $S$ which itself are allowed to be very large, but with essentially large fibres $X_{s} \subset X$ for all $s \in S$.

As a convention from now on, we consider all $\infty$-categories to be large, unless we explicitly allow them to be very large. 
Lemma A.2. For every (large) $\infty$-category $C$ the assignment $c \mapsto C_{/ c}$ defines a functor $C \rightarrow$ Cat $_{\infty}$. Picking for every $c \in C$ the object $\mathrm{id}_{c} \in C_{/ c}$ as the basepoint this functor even refines to a functor $C \rightarrow\left(\mathcal{C a t}_{\infty}\right)_{\star}$.

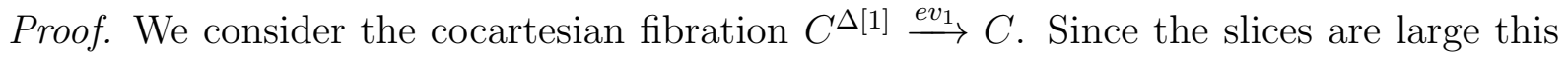
fibration is classified by a functor $C \rightarrow \mathcal{C} t_{\infty}$. Thus there is a pullback diagram

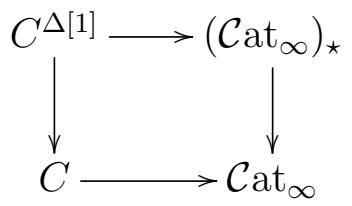

Now we take the canonical section $\operatorname{id}_{(-)}: C \rightarrow C^{\Delta[1]}$ and obtain the functor $C \rightarrow\left(\mathcal{C a t}_{\infty}\right)_{\star}$ as a composition.

By definition of $\operatorname{Gr} \mathcal{R}$ ing $\mathcal{S}$ p (see Definition 2.4) there is a pullback diagram

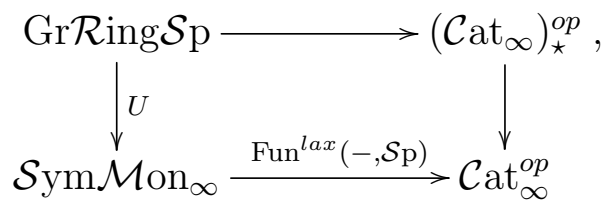

where $\mathcal{S} y m \mathcal{M} \mathrm{Mn}_{\infty}$ denotes the very large category of (large) symmetric monoidal categories and lax symmetric monoidal functors. We denote the left vertical functor that associates to every graded ring spectrum its underlying grading symmetric monoidal $\infty$ category by $U$. Here the right vertical morphism is just the opposite of the universal cocartesian fibration, hence the universal cartesian fibration.

Note that for an arbitrary (large) $\infty$-category $C$, the presheaf category $\operatorname{Fun}\left(C^{o p}, \mathcal{S p}\right)$ becomes a symmetric monoidal $\infty$-category such that evaluation for every object $c \in$ $C$ is a monoidal functor $\operatorname{Fun}\left(C^{o p}, \mathcal{S}\right.$ p) $\rightarrow \mathcal{S}$ p. Thus for any object $c \in C$ the pair $\left(\right.$ Fun $\left(C^{o p}, \mathcal{S}\right.$ p),$\left.e v_{c}\right)$ defines a graded ring spectrum. We now want to show that this assignment is even functorial in $(C, c)$.

Lemma A.3. There is a functor

$$
\left(\mathcal{C a t}_{\infty}\right)_{\star}^{o p} \rightarrow \operatorname{Gr} \mathcal{R i n g} \mathcal{S p} \quad(C, c) \mapsto\left(\operatorname{Fun}\left(C^{o p}, \mathcal{S p}\right), e v_{c}\right)
$$

Proof. Let $g: \mathcal{C}_{\mathrm{at}_{\infty}}^{o p} \rightarrow \mathcal{S}$ ym $\mathcal{M}$ on $_{\infty}$ be any functor. Since Gr $\mathcal{R}$ ing $\mathcal{S}$ p fits into the pullback diagram 43 a functor $f:\left(\mathcal{C}_{\text {at }}^{\infty}\right)_{\star}^{o p} \rightarrow \operatorname{Gr} \mathcal{R}$ ing $\mathcal{S}$ p covering $g$ is then uniquely determined (up to a contractible space of choices) by a commuting diagram

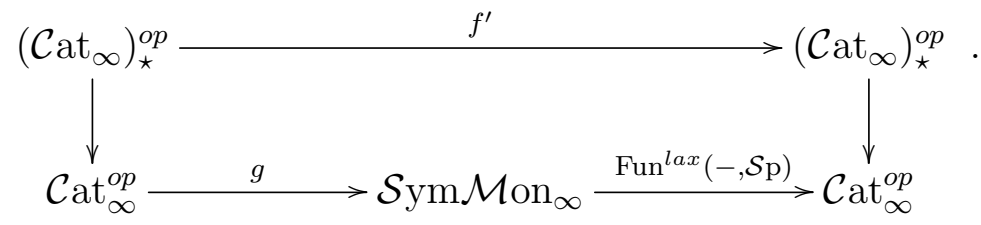


For our concrete construction we define the functor $g$ by $g(C):=\operatorname{Fun}(C, \mathcal{S p})$. Since the right vertical morphism in the diagram is the universal cocartesian fibration, a functor $f^{\prime}$ that makes the diagram commute can be written as a functor into the fibration classfied by the lower horizontal composition $g^{\prime}$. Moreover the source of $f^{\prime}$ is itself a Grothendieck construction, described by the identity functor. Thus the functor $f^{\prime}$ (and therefore also the original functor $f$ ) can equivalently be described as a transformation of functors id $\Rightarrow g^{\prime}$ from $\mathcal{C}$ at ${ }_{\infty}$ to itself. Such a transformation is given as follows: for every $\infty$-category $C$ it is the assignment

$$
C \rightarrow \operatorname{Fun}^{l a x}(\operatorname{Fun}(C, \mathcal{S p}), \mathcal{S p}) \quad c \mapsto e v_{c}
$$

which can be easily seen to exist functorially in $C$ using the definitions of the functor categories.

Let now $\mathcal{M}$ be an arbitrary, essentially small $\infty$-category, for example the category of smooth manifolds. Then for every object $M \in \mathcal{M}$ we define the category of presheaves of spectra on $M$ as

$$
\operatorname{PSh}_{\mathcal{S}_{\mathrm{p}}}(M):=\operatorname{Fun}\left(\left(\mathcal{M}_{/ M}\right)^{o p}, \mathcal{S p}\right)
$$

which becomes a symmetric monoidal $\infty$-category with the pointwise tensor product. There is a global sections functor

$$
\Gamma(\bullet, M): \operatorname{PSh}_{\mathcal{S}}(M) \rightarrow \mathcal{S p}
$$

which is in our case just evaluation at the identity of $M$. The last two lemmas imply the following functoriality statement.

Corollary A.4. The assignment $M \mapsto\left(\mathrm{PSh}_{\mathcal{S} \mathrm{p}}(M), \Gamma(\bullet, M)\right)$ refines to a functor

$$
\widehat{\mathrm{PSh}_{\mathcal{S}}}: \quad \mathcal{M}^{o p} \rightarrow \operatorname{Gr} \mathcal{R i n g} \mathcal{S p}
$$

Proof. We apply Lemma A.2 with $\mathcal{C}=\mathcal{M}$ and obtain a functor $\mathcal{M} \rightarrow\left(\mathcal{C a t}_{\infty}\right)_{\star}$. Then we take the opposite and compose this functor with the functor $\left(\mathcal{C} \text { at }{ }_{\infty}\right)_{\star}^{o p} \rightarrow \operatorname{Gr} \mathcal{R}$ ing $\mathcal{S}$ p from Lemma A.3.

The last corollary establishes the basic example of a presheaf of graded ring spectra. We now want to show how to derive new examples from this one.

Proposition A.5 (Change of grading). Assume that we have an $\infty$-category $\mathcal{D}$ together with functors

$$
F: \mathcal{D} \rightarrow \operatorname{Gr} \operatorname{Ring} \mathcal{S p}, \quad G: \mathcal{D} \rightarrow \mathcal{S y m}_{\mathrm{yon}} \mathcal{M o n}_{\infty}
$$

and a transformation $\eta$ from $G$ to the composition $\mathcal{D} \stackrel{F}{\rightarrow} \operatorname{Gr} \mathcal{R} \operatorname{ing} \mathcal{S} \mathrm{p} \stackrel{U}{\rightarrow} \mathcal{S} \mathrm{ym}_{\mathcal{M}} \mathrm{Mn}_{\infty}$. Then there is a functor

$$
\eta^{*} F: \quad \mathcal{D} \rightarrow \operatorname{Gr} \mathcal{R i n g} \mathcal{S} \mathrm{p} \quad d \mapsto(G(d), G(d) \stackrel{\eta}{\rightarrow} U(F(d)) \stackrel{F(d)}{\longrightarrow} \mathcal{S} \mathrm{p}) .
$$


Proof. We write the transformation $\eta$ as a morphism $\mathcal{D} \times \Delta[1] \rightarrow \mathcal{S y m}_{\mathcal{M}} \mathrm{Mn}_{\infty}$. Then we have a commuting square

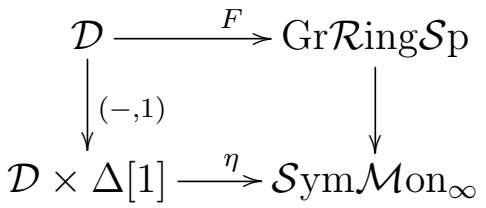

We want to show that there is a lift $L: \mathcal{D} \times \Delta[1] \rightarrow \operatorname{Gr} \mathcal{R}$ ing $\mathcal{S}$ p in the diagram with the property that for every $d \in \mathcal{D}$ the edge $L\left(i \mathrm{~d}_{d}, 0 \rightarrow 1\right)$ is cartesian. Then the desired functor is given by the restriction of $L$ to $\mathcal{D} \times 0$.

Now we want to show that such a lift $L$ exists. This is equivalent to a cartesian lift in the diagram

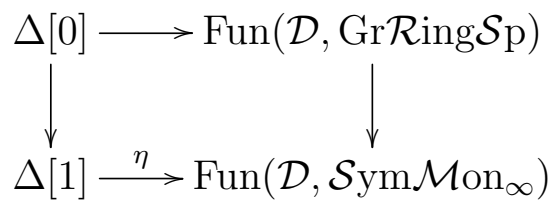

But the right vertical morphism is a cartesian fibration since $\operatorname{Gr} \mathcal{R}$ ing $\mathcal{S} p \rightarrow \mathcal{S}_{\mathrm{pm}} \mathcal{M} \mathrm{Mn}_{\infty}$ is one by definition and exponentials of cartesian fibrations are again cartesian fibrations as shown in [Lur09, Proposition 3.1.2.1]. Thus there is an essentially unique cartesian lift as desired.

Remark A.6. Let $\mathcal{M}$ be an $\infty$-category. From Corollary A.4 we have an object $\mathrm{PSh}_{\mathcal{S}_{\mathrm{p}}} \in$ $\operatorname{Fun}\left(\mathcal{M}^{o p}, \mathcal{S}_{y m} \mathcal{M o n}_{\infty}\right)$ together with a lift $\widehat{\operatorname{PSh}_{\mathcal{S}}} \in \operatorname{Fun}\left(\mathcal{M}^{o p}, \operatorname{Gr} \mathcal{R i n g} \mathcal{S} \mathrm{p}\right)$. Together with the change of grading statement we can obtain new examples of functors

$$
\eta^{*} \widehat{\mathrm{PSh}_{\mathcal{S}}} \in \operatorname{Fun}\left(\mathcal{M}^{o p}, \operatorname{Gr} \operatorname{Ring} \mathcal{S p}\right)
$$

from a natural transformation $\eta: G \rightarrow \operatorname{PSh}_{\mathcal{S} \text { p }}$ for any functor $G \in \operatorname{Fun}\left(\mathcal{M}^{o p}, \mathcal{S}_{\text {ym }} \mathcal{M}_{\infty}\right)$. All examples in this paper will arise in this way. This is not a coincidence, since one can show that all examples of functors in $\operatorname{Fun}\left(\mathcal{M}^{o p}, \operatorname{Gr} \mathcal{R i n g} \mathcal{S}\right.$ p) can be constructed from $\mathrm{PSh}_{\mathcal{S} \mathrm{p}}$ using the change of grading statement. In other words the object $\widehat{\mathrm{PSh}_{\mathcal{S}}}$ over $\mathrm{PSh}_{\mathcal{S} \mathrm{p}}$ is the universal presheaf of graded ring spectra over $\mathcal{M}$. We refrain from giving the proof here since it is technically involved and we do not need the statement.

Lemma A.7. We assume that $I$ is a small $\infty$-category and let $v \in I^{\triangleleft}$ be the cone point. Then every functor $F: I^{\triangleleft} \rightarrow \operatorname{Gr} \mathcal{R i n g} \mathcal{S} \mathrm{p}$ induces a functor $\tilde{F}: I^{\triangleleft} \rightarrow \operatorname{Gr} \mathcal{R} \operatorname{ing} \mathcal{S} \mathrm{p}_{U(F(v))}$. Hence for every $c \in U\left(F(v)\right.$ ) (see (43) for $U$ ) we obtain a functor $\tilde{F}^{c}: I^{\triangleleft} \rightarrow \mathcal{S}$.

Proof. Apply the change of grading Proposition A.5 to the functor $F$, the constant functor $G: I^{\triangleleft} \rightarrow \mathcal{S y m}_{\mathrm{yon}_{\infty}}$ with image $U(F(v)) \in \mathcal{S}_{\mathrm{ym}} \mathcal{M} \mathrm{Mon}_{\infty}$, and the obvious natural transformation $G \rightarrow U \circ F$. Explicitly this transformation is given as the composition

$$
\eta: \quad I^{\triangleleft} \times \Delta[1] \rightarrow I^{\triangleleft} \rightarrow \operatorname{Gr} \mathcal{R i n g} \mathcal{S p} \rightarrow \mathcal{S y m}_{\operatorname{Mon}}
$$

where the first functor is the identity on $I^{\triangleleft} \times\{1\}$ and carries $I^{\triangleleft} \times\{0\}$ to the cone point. 
Now we want to give a short discussion of limits of graded ring spectra. This will be important when imposing descent conditions for presheaves of graded ring spectra. The following result is essentially due to Lurie [Lur09, Section 4.3.1] and holds more generally, but we state it explicitly in the form needed below.

Proposition A.8. 1. The $\infty$-category $\operatorname{Gr} \mathcal{R} i n g \mathcal{S p}$ admits limits and the projection $\operatorname{Gr} \mathcal{R i n g} \mathcal{S} p \rightarrow \mathcal{S y m}_{\mathrm{M}} \mathcal{M n}_{\infty}$ preserves limits.

2. A diagram $F: I^{\triangleleft} \rightarrow \operatorname{Gr} \mathcal{R}$ ing $\mathcal{S} \mathrm{p}$ is a limit diagram if and only if the following conditions are satisifed:

- The induced diagram $I^{\triangleleft} \rightarrow \operatorname{Gr} \mathcal{R i n g} \mathcal{S} \mathrm{p} \rightarrow \mathcal{S y m}_{\mathcal{M}} \mathcal{M o n}_{\infty}$ is a limit diagram

- For every object $c \in U(F(v))$ the resulting diagram $\tilde{F}^{c}: I^{\triangleleft} \rightarrow \mathcal{S} \mathrm{p}$ (see Lemma A.7) is a limit diagram

Proof. 1.) We first observe that the functor $U: \operatorname{Gr} \mathcal{R i n g} \mathcal{S} \mathrm{p} \rightarrow \mathcal{S y m}_{\mathcal{M}} \mathcal{M o n}_{\infty}$ admits a left adjoint given by the functor $\mathcal{S}$ ym $\mathcal{M}$ on $_{\infty} \rightarrow \operatorname{Gr} \mathcal{R}$ ing $\mathcal{S}$ p that maps every $C$ to the initial object of the fibre $\operatorname{Gr} \mathcal{R}$ ing $\mathcal{S} \mathrm{p}_{C}=\operatorname{Fun}_{\text {lax }}^{\otimes}(C, \mathcal{S} \mathrm{p})$, see [Lur09, Proposition 2.4.4.9] for the existence of this functor and [Lur09, Proposition 5.2.4.3] for the fact that it is left adjoint to $U$. This shows that $U$ preserves all limits.

We now want to show that $\operatorname{Gr} \mathcal{R}$ ing $\mathcal{S}$ p has all limits. It follows from Lur09, Remark 4.3.1.5] that a diagram $F: I^{\triangleleft} \rightarrow$ GrRing $\mathcal{S}$ p is a limit diagram if $I^{\triangleleft} \rightarrow$ GrRing $\mathcal{S}$ p $\rightarrow$ $\mathcal{S y m}_{\mathcal{M}} \mathcal{M o n}_{\infty}$ is a limit diagram and $F$ is a $U$-limit diagram (this is a relative limit as discussed in [Lur09, section 4.3.1]). Thus using the fact that $\mathcal{S}_{y m} \mathcal{M}$ on $_{\infty}$ has all limits we conclude that we can extend any diagram $I \rightarrow \operatorname{Gr} \mathcal{R} i n g \mathcal{S}$ p to a square

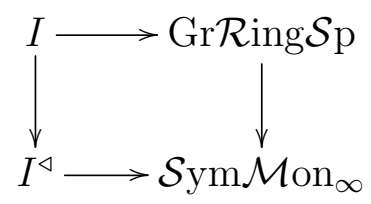

where the lower horizontal morphism is a limit diagram. Then we need to show that we can find a lift $I^{\triangleleft} \rightarrow \operatorname{Gr} \mathcal{R}$ ing $\mathcal{S}$ p in this diagram that is a $U$-limit. But the existence of such a lift is shown in [Lur09, Corollary 4.3.1.11] under the condition that

- The fibres GrRing $\mathcal{S p}_{C}=\operatorname{Fun}_{\text {lax }}^{\otimes}\left(C, \mathcal{S}\right.$ p) have all limits for all $C \in \mathcal{S} \mathrm{ym}_{\mathcal{M}} \mathcal{M n}_{\infty}$.

- The functor $\operatorname{Gr} \mathcal{R} \operatorname{ing} \mathcal{S} \mathrm{p}_{C} \rightarrow \operatorname{Gr} \mathcal{R}$ ing $\mathcal{S} \mathrm{p}_{C^{\prime}}$ associated to a morphism $C^{\prime} \rightarrow C$ in $\mathcal{S y m}_{\mathcal{M}} \mathrm{Mon}_{\infty}$ preserves all limits.

These conditions are satisfied as shown in [Lur11, Section 3.2.2].

2) Assume $F: I^{\triangleleft} \rightarrow \operatorname{Gr} \mathcal{R}$ ing $\mathcal{S}$ p is given. The above discussion shows that the assertion that $F$ is a limit diagram is equivalent to

- The induced diagram $I^{\triangleleft} \rightarrow \operatorname{Gr} \mathcal{R} \operatorname{ing} \mathcal{S} \mathrm{p} \rightarrow \mathcal{S}_{\mathrm{ym}} \mathcal{M}_{\infty}$ is a limit diagram 
- The diagram $F$ is a $U$-limit diagram.

We now want to show that the second condition ( $F$ is a $U$-limit diagram) is equivalent to the second condition of the proposition. Therefore we claim that it follows from Lur09, Proposition 4.3.1.9.] that this condition is equivalent to the condition:

- The diagram $\tilde{F}: I^{\triangleleft} \rightarrow \operatorname{Gr} \mathcal{R} \operatorname{ing} \mathcal{S} \mathrm{p}_{U(F(v))}$ (see Lemma A.7) is a limit diagram.

To see this note that $\tilde{F}$ comes by construction with a natural tranformation $\tilde{F} \rightarrow F$ which satisifies the assumption of [Lur09, Proposition 4.3.1.9.]. Now we only need to observe that limits in

$$
\operatorname{Gr} \operatorname{Ring} \mathcal{S} \mathrm{p}_{U(F(v))}=\operatorname{Fun}_{\operatorname{lax}}^{\otimes}(U(F(v)), \mathcal{S} \mathrm{p})
$$

are computed pointwise, i.e. they can be detected by evaluation at each $c \in U(F(v))$

Let $\mathcal{M}$ be a Grothendieck site, i.e. an $\infty$-category equipped with a Grothendieck topology on its homotopy category $\mathcal{H} \mathrm{o}(\mathcal{M})$. In Corollary A.4 we constructed a presheaf of graded ring spectra $\widehat{\mathrm{PSh}_{\mathcal{S}}}$. Now we want to discuss a variant and consider for $M \in \mathcal{M}$ the full subcategory

$$
\operatorname{Sh}_{\mathcal{S p}}(M) \subset \operatorname{PSh}_{S p}(M)
$$

of sheaves on the overcategory $\mathcal{M} / M$ which also becomes a site when equipped with the induced Grothendieck topology. This defines a presheaf of symmetric monoidal categories $\mathrm{Sh}_{\mathcal{S} \mathrm{p}} \in \mathrm{PSh}_{\mathcal{S}_{\mathrm{ym}} \mathcal{M} \text { on }_{\infty}}(\mathcal{M})$. Then the change of grading statement immediately allows to refine this to a presheaf of graded ring spectra

$$
\widehat{\operatorname{Sh}_{\mathcal{S p}}} \in \operatorname{PSh}_{\mathrm{Gr} \mathcal{R} \operatorname{ing} \mathcal{S} \mathrm{p}}(\mathcal{M})
$$

We now want to show that this assignment is a sheaf of graded ring spectra. First note that $\mathrm{PSh}_{\mathcal{S} \mathrm{p}}$ and $\mathrm{Sh}_{\mathcal{S} \mathrm{p}}$ both form sheaves of symmetric monoidal categories as opposed to presheaves.

Proposition A.9. Let $\mathcal{M}$ be a Grothendieck site.

1. The assignment $M \mapsto\left(\operatorname{Sh}_{\mathcal{S}}(M), \Gamma(\bullet, M)\right)$ refines to a sheaf of graded ring spectra on $\mathcal{M}$ :

$$
\widehat{\operatorname{Sh}_{\mathcal{S p}}} \in \operatorname{Sh}_{\text {GrRing } \mathcal{P}}(\mathcal{M})
$$

2. Let $F: \mathcal{M}^{o p} \rightarrow$ GrRing $\mathcal{S p}$ be a sheaf of graded ring spectra, $G: \mathcal{M}^{o p} \rightarrow \mathcal{S}_{\mathrm{ym}} \mathcal{M}_{\infty}$ be a sheaf of symmetric monoidal categories, and $\eta: G \rightarrow U \circ F$ be a natural transformation. Then the functor $\eta^{*} F: \mathcal{M}^{o p} \rightarrow \operatorname{Gr} \mathcal{R i n g} \mathcal{S} \mathrm{p}$ obtained by change of grading is also a sheaf of graded ring spectra.

3. The functor

$$
\operatorname{Sh}_{\text {GrRing } \mathcal{S} p}(\mathcal{M}) \rightarrow \operatorname{Sh}_{\mathcal{S y m}_{\mathcal{M}} \operatorname{Mon}_{\infty}}(\mathcal{M})
$$

induced by $U$ is a cartesian fibration. 
Proof. In order to show (1) let $M \in \mathcal{M}$ be an object and $\left\{U_{i} \rightarrow M\right\}_{i \in I}$ be a covering family. Forming the Čech nerve and applying the functor $\widehat{\mathrm{Sh}_{\mathcal{S p}}}$ we obtain the augmented cosimplicial object

$$
\widehat{\operatorname{Sh}_{\mathcal{S p}}}\left(U_{\bullet}\right): \quad \Delta^{\triangleleft} \rightarrow \operatorname{Gr} \mathcal{R} \text { ing } \mathcal{S} \text { p } .
$$

We have to show that this augmented cosimplicial object is a limit diagram. According to Proposition A.8 we have to check that

- The induced diagram $\operatorname{Sh}_{\mathcal{S}}\left(U_{\bullet}\right): \Delta^{\triangleleft} \rightarrow \mathcal{S y m}_{\mathcal{M}} \operatorname{Mon}_{\infty}$ is a limit diagram.

- For every object $c \in \operatorname{Sh}(M)$ the resulting diagram $c\left(U_{\bullet}\right): I^{\triangleleft} \rightarrow \mathcal{S}$ p is a limit diagram.

The first condition is precisely the sheaf condition for $\mathrm{Sh}_{\mathcal{S p}}: \mathcal{M}^{o p} \rightarrow \mathcal{S}_{\mathrm{ym}} \mathcal{M}_{\infty} n_{\infty}$ and therefore true as remarked before the Proposition. The second condition is precisely the sheaf condition for $c:(\mathcal{M} / M)^{o p} \rightarrow \mathcal{S}$ p and is also satisified since $c$ is a sheaf.

Now we want to show (2). The proof works similar to the proof of (1). One has to show that a diagram

$$
\eta^{*} F\left(U_{\bullet}\right): \quad \Delta^{\triangleleft} \rightarrow \operatorname{Gr} \mathcal{R} \operatorname{ing} \mathcal{S} \text { p. }
$$

is a limit diagram. In this case the two diagrams that have to be identified as limits according to Proposition A.8 are

$$
G\left(U_{\bullet}\right): \Delta^{\triangleleft} \rightarrow \mathcal{S y m}_{\operatorname{Mon}} \quad \text { and } \quad \tilde{F}^{d}\left(U_{\bullet}\right): \Delta^{\triangleleft} \rightarrow \mathcal{S} \mathrm{p}
$$

for $c \in G(M)$ and $d=\eta_{M}(c) \in U\left(F(M)\right.$ ) (see A.7 for the notation $\tilde{F}^{d}$ ). The first follows from the fact that $G$ is a sheaf and the second from the fact that $F$ was a sheaf.

Finally for (3) first note that the induced functor

$$
U_{*}: \operatorname{PSh}_{\text {GrRing } \mathcal{S}}(\mathcal{M}) \rightarrow \operatorname{PSh}_{\mathcal{S y m}_{\operatorname{Mon}}}(\mathcal{M})
$$

restricts to a functor of sheaves

$$
\hat{U}_{*}: \operatorname{Sh}_{\text {GrRing } \mathcal{S}}(\mathcal{M}) \rightarrow \operatorname{Sh}_{\mathcal{S}_{\text {ym } \mathcal{M}} o_{\infty}}(\mathcal{M})
$$

as stated since $U$ preserves limits, as shown in Proposition A.8. The next step is to observe that the inclusion $i: \operatorname{Sh}_{\mathrm{GrRing} \mathcal{S} \mathrm{p}}(\mathcal{M}) \rightarrow \operatorname{PSh}_{\mathrm{Gr} \mathcal{R} \text { ing } \mathcal{S}_{\mathrm{p}}}(\mathcal{M})$ has the following property:

Let $e: \Delta[1] \rightarrow \operatorname{Sh}_{\mathrm{GrRing} \mathcal{S}}(\mathcal{M})$ be an edge such that $i(e)$ is $U_{*}$-cartesian. Then $e$ is $\hat{U}_{*}$-cartesian.

This basically follows since the inclusion of sheaves into presheaves is full. To show that $\hat{U}_{*}$ is a cartesian fibration we have to show that $\hat{U}_{*}$ is an inner fibration and every edge in $\operatorname{Sh}_{\mathcal{S} y m \mathcal{M}{ }_{\infty}}(\mathcal{M})$ with a given lift of the target has a $\hat{U}_{*}$-cartesian lift to $\operatorname{Sh}_{\text {GrRingS }}(\mathcal{M})$. We observe that $\hat{U}_{*}$ is an inner fibration since the inlcusion of presheaves into sheaves is full and $U_{*}$ is an inner fibration. For the lifting property we use statement (2) of the Proposition to lift to an edge $e$ in $\operatorname{Sh}_{\mathrm{Gr} R \text { ing } \mathcal{S} \mathrm{p}}(\mathcal{M})$ whose image $i(e)$ is $U_{*}$ cartesian. Then we can conclude that $e$ is $\hat{U}_{*}$-cartesian as remarked above. 


\section{B Locally constant sheaves}

Let $\mathcal{C}$ be a presentable $\infty$-category and $M$ be a smooth manifold. Recall that a $\mathcal{C}$-valued sheaf on a manifold $M$ (more precisely the site $\mathcal{M f} / M$ ) is called constant when it is equivalent to the restriction $\left(\hat{C}_{X}\right)_{\mid M}$ of a constant sheaf $\hat{C}_{X} \in \mathrm{Sh}_{\mathcal{C}}$ for an object $X \in \mathcal{C}$. Also recall that we have an equivalence of $\hat{C}_{X}$ with the sheaf

$$
\underline{X} \in \mathrm{Sh}_{\mathcal{C}}, \quad M \mapsto X^{\Pi_{\infty}(M)}
$$

(see Remark B.3 for the notation $\Pi_{\infty}(M)$ ) as shown in [BG13, Lemma 6.7], see Proposition 3.3. A locally constant sheaf was defined in Section 3 as follows:

Definition B.1. A sheaf $\mathcal{F} \in \operatorname{Sh}_{\mathcal{C}}(M)$ is called locally constant if every point $m \in M$ has a neighbourhood $U \subseteq M$ such that $\mathcal{F}_{\mid U} \in \operatorname{Sh}_{\mathcal{C}}(U)$ is constant. We write

$$
\operatorname{Sh}_{\mathcal{C}}^{\text {loc }}(M) \subseteq \operatorname{Sh}_{\mathcal{C}}(M)
$$

for the full subcategory of locally constant sheaves.

We want to prove the following statement, which is a $\infty$-categorical generalization of the classification of local systems using covering theory:

Theorem B.2. There is an equivalence of $\infty$-categories $\operatorname{Sh}_{\mathcal{C}}^{\text {loc }}(M) \simeq \operatorname{Fun}\left(\Pi_{\infty}(M), \mathcal{C}\right)$ which is natural in $M$.

Remark B.3. - The $\infty$-groupoid $\Pi_{\infty}(M)$ is the simplicial version of the fundamental$\infty$-groupoid of the space $M$. Its homotopy category is the ordinary fundamental groupoid $\mathcal{H} \mathrm{o}\left(\Pi_{\infty}(M)\right) \simeq \Pi_{1}(M)$. Therefore if $\mathcal{C}$ is a 1-category, Theorem B.2 reduces to the well-known classification of ordinary local systems.

- If $\mathcal{C}$ is symmetric monoidal, then the equivalence is compatible with the induced monoidal structures.

We now want to prove Theorem B.2 in several steps.

Lemma B.4. - The presheaves

$$
\operatorname{Sh}_{\mathcal{C}}^{\text {loc }}(-): \mathcal{M f} f^{o p} \rightarrow \mathcal{C} \text { at }_{\infty} \quad \text { and } \operatorname{Fun}\left(\Pi_{\infty}(-), \mathcal{C}\right): \mathcal{M f} f^{o p} \rightarrow \mathcal{C a t}_{\infty}
$$

are sheaves of $\infty$-categories on the site of smooth manifolds.

- There are functors $F_{M}: \operatorname{Fun}\left(\Pi_{\infty}(M), \mathcal{C}\right) \rightarrow \operatorname{Sh}_{\mathcal{C}}^{\text {loc }}(M)$ which are natural in $M$ such that the diagram

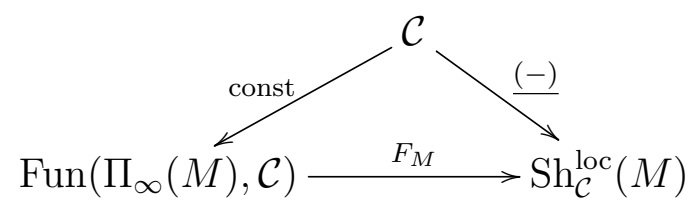

commutes for every $M$. 
Proof. To see that $\mathrm{Sh}_{\mathcal{C}}^{\text {loc }}(-)$ is a sheaf it suffices to observe that $\mathrm{Sh}_{\mathcal{C}}(-)$ is a sheaf and the condition of being locally constant is a local condition. For the second statement we consider the category $\mathcal{C} \in \mathcal{C}$ at $t_{\infty}$ as an object in the (very large) presentable category of (large) $\infty$-categories. Then $\mathcal{C}$ induces the constant sheaf $\hat{C}_{\mathcal{C}} \in \mathrm{Sh}_{\mathcal{C}^{+} t_{\infty}}$. As we have remarked above (for general target categories) it is equivalent to the sheaf $\operatorname{Fun}\left(\Pi_{\infty}(-), \mathcal{C}\right) \in \mathrm{Sh}_{\mathcal{C}^{+} \infty}$. In particular, $\operatorname{Fun}\left(\Pi_{\infty}(-), \mathcal{C}\right)$ is constant. In order to construct a transformation

$$
\operatorname{Fun}\left(\Pi_{\infty}(-), \mathcal{C}\right) \rightarrow \operatorname{Sh}_{\mathcal{C}}^{\text {loc }}(-)
$$

it therefore suffices to construct a transformation of $\infty$-category valued sheaves

$$
\hat{C}_{\mathcal{C}} \rightarrow \mathrm{Sh}_{\mathcal{C}}^{\text {loc }}
$$

Such a transformation is clearly given by the transformation of presheaves

$$
\mathcal{C} \cong C_{\mathcal{C}}(M) \rightarrow \operatorname{Sh}_{\mathcal{C}}^{\text {loc }}(M) \quad C \mapsto \underline{C} .
$$

for every $M$.

Remark B.5. The proof of Lemma B.4 shows that the sheaf $\operatorname{Fun}\left(\Pi_{\infty}(-), \mathcal{C}\right)$ is constant as a sheaf of categories. Thus the equivalence $\operatorname{Fun}\left(\Pi_{\infty}(M), \mathcal{C}\right) \cong \operatorname{Sh}_{\mathcal{C}}^{\text {loc }}(M)$ (which still has to be proven) shows that also $\operatorname{Sh}_{\mathcal{C}}^{\text {loc }}(-)$ is constant as a sheaf of categories. This underlines the general philosphy that locally constant objects are sections in a constant sheaf.

As a next step we need a technical lemma about limits in $\mathcal{C}_{a}$.

Lemma B.6. Assume we have two cosimplicial $\infty$-categories $C_{\bullet}$ and $D$. (i.e. functors $\left.\Delta \rightarrow \mathcal{C}_{a_{\infty}}\right)$ together with a cosimplicial functor $F_{\bullet}: C_{\bullet} \rightarrow D_{\bullet}$ such that $F_{i}$ is fully faithful for each $i \in \Delta$. Then we have the following assertions:

1. The functor $\lim _{\Delta} C_{\bullet} \rightarrow \lim _{\Delta} D_{\bullet}$ is fully faithful

2. The diagram

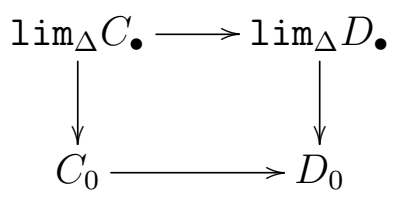

is a pullback in the $\infty$-category $\mathcal{C}_{\infty}$.

Proof. The first part is true for any limit, not just limits indexed by $\Delta$. This follows from the observation that a functor $F: C \rightarrow D$ is fully faithful precisely if the diagram

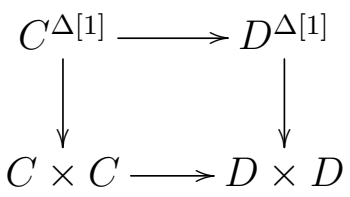


is a pullback. If $F$ is a limit of functors $F_{i}$ then this diagram is the limit of the corresponding diagrams for the functors $F_{i}$ since all operations commute with limits. Therefore if all the $F_{i}$-diagrams are pullbacks it follows that also the limit diagram is a pullback.

To show the pullback property we want to show that the natural map

$$
\varphi: \lim _{\Delta} C_{\bullet} \rightarrow C_{0} \times_{D_{0}} \lim _{\Delta} D \bullet
$$

is an equivalence. Therefore we first note that the functor $C_{0} \times_{D_{0}} \lim _{\Delta} D_{\mathbf{0}} \rightarrow \lim _{\Delta} D$. is fully faithful as the pullback of a fully faithful functor. Also from the first assertion we know that $\lim _{\Delta} C_{\bullet} \rightarrow \lim _{\Delta} D$ • is fully faithful. Thus we really compare two full subcategories of $\lim _{\Delta} D$. and it suffices to compare their essential images. This means that it suffices to check that the fibre of

$$
\lim _{\Delta} F_{\bullet}: \lim _{\Delta} C_{\bullet} \rightarrow \lim _{\Delta} D_{\bullet}
$$

at every object of $\lim _{\Delta} D$. is equivalent to the fibre of

$$
F_{0}: C_{0} \rightarrow D_{0}
$$

The fibre of $\lim _{\Delta} F_{\bullet}$ is given by the limit of the fibres of the $F_{i}^{\prime} s$, hence a limit of categories which are either a singleton or empty (since the $F_{i}$ are fully faithful). This limit is either empty or a singleton depending on whether the corresponding diagram consists entirely of empty categories or singletons. In particular it is empty if and only if the fibre of $F_{0}: C_{0} \rightarrow D_{0}$ is empty. This implies the second assertion of the lemma.

Corollary B.7. We assume $F$ and $G$ are sheaves of $\infty$-categories on some Grothendieck site $\mathcal{M}$ and $\eta: F \rightarrow G$ is a morphism of sheaves such that $\eta_{M}: F(M) \rightarrow G(M)$ is fully faithful for every element $M \in \mathcal{M}$. Then for every covering family $\left\{U_{i} \rightarrow M\right\}_{i \in I}$ the diagram

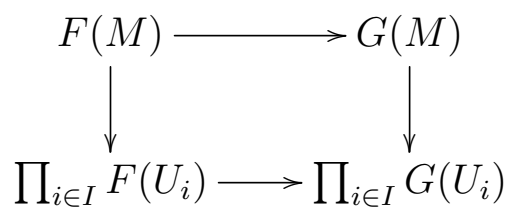

is a pullback diagram in $\mathcal{C} \mathrm{t}_{\infty}$. In particular an object $x \in G(M)$ lies in the essential image of $\eta_{M}: F(M) \rightarrow G(M)$ precisely if there exist a covering family $\left\{U_{i} \rightarrow M\right\}_{i \in I}$ such that for every $i \in I$ the restriction $\left.x\right|_{U_{i}} \in G\left(U_{i}\right)$ lies in the essential image of $\eta_{U_{i}}: F\left(U_{i}\right) \rightarrow G\left(U_{i}\right)$.

Proof of Theorem B.2. We want to show that the functors $F_{M}: \operatorname{Fun}\left(\Pi_{\infty}(M), \mathcal{C}\right) \rightarrow$ $\operatorname{Sh}_{\mathcal{C}}^{\text {loc }}(M)$ constructed in Lemma B.4 are equivalences for every $M$. We first assume that $M$ is contractible. Then using Diagram (44) we see that the functor $F_{M}$ is equivalent to the functor

$$
\mathcal{C} \rightarrow \operatorname{Sh}_{\mathcal{C}}^{\text {loc }}(M) \quad C \mapsto \underline{C} .
$$


This functor is clearly fully faithful since one easily computes

$$
\begin{aligned}
\operatorname{Map}_{\mathrm{Sh}_{\mathcal{C}}^{\text {loc }}(M)}(\underline{C}, \underline{D}) \simeq \operatorname{Map}_{\mathrm{Sh}_{\mathcal{C}}(M)}(\underline{C}, \underline{D}) & \simeq \operatorname{Map}_{\mathrm{PSh}_{\mathcal{C}}(M)}\left(\operatorname{const}_{C}, \underline{D}\right) \\
& \simeq \operatorname{Map}_{\mathcal{C}}\left(C, D_{\infty} \Pi_{\infty}\right) \\
& \simeq \operatorname{Map}_{\mathcal{C}}(C, D) .
\end{aligned}
$$

Thus we know that the functor $F_{M}$ is fully faithful for contractible $M$.

For arbitrary $M$ we can choose a good open cover $\left\{U_{i}\right\}$, i.e. an open cover that has the property that all finite intersections $U_{i_{1}, \ldots, i_{k}}:=U_{i_{1}} \cap \ldots \cap U_{i_{k}}$ are contractible. Then using the fact that $\operatorname{Fun}\left(\Pi_{\infty}(-), \mathcal{C}\right)$ and $\operatorname{Sh}_{\mathcal{C}}^{\text {loc }}(-)$ are sheaves as established in B.4 we can write the functor $F_{M}$ as the limit of functors

$$
F_{U_{i_{1}, \ldots, i_{k}}}: \quad \operatorname{Fun}\left(\Pi_{\infty}\left(U_{i_{1}, \ldots, i_{k}}\right), \mathcal{C}\right) \rightarrow \operatorname{Sh}_{\mathcal{C}}^{\text {loc }}\left(U_{i_{1}, \ldots, i_{k}}\right)
$$

Using that all these functors are fully faithful since $U_{i_{1}, \ldots, i_{k}}$ is contractible and the first claim of lemma B.6 we can conclude that $F_{M}$ is also fully faithful for any $M$.

Finally we want to show that $F_{M}: \operatorname{Fun}\left(\Pi_{\infty}(M), \mathcal{C}\right) \rightarrow \operatorname{Sh}_{\mathcal{C}}^{\text {loc }}(M)$ is essentially surjective. Therefore let $\mathcal{F} \in \operatorname{Sh}_{\mathcal{C}}^{\text {loc }}(M)$. Since $\mathcal{F}$ is locally constant we find an open cover $\left\{U_{i}\right\}$ of $M$ such that $\left.\mathcal{F}\right|_{U_{i}}$ is constant. But constant sheaves clearly lie in the essential image of the functors $F_{U_{i}}: \operatorname{Fun}\left(\Pi_{\infty}\left(U_{i}\right), \mathcal{C}\right) \rightarrow \operatorname{Sh}_{\mathcal{C}}^{\text {loc }}\left(U_{i}\right)$ which can be seen from Diagram (44). But then Corollary B.7 implies that $\mathcal{F}$ lies in the essential image of $F_{M}$.

Corollary B.8. Every locally constant sheaf $\mathcal{F} \in \operatorname{Sh}_{\mathcal{C}}^{\text {loc }}(M)$ is homotopy invariant, i.e. for a morphism $N \stackrel{f}{\rightarrow} N^{\prime} \rightarrow M$ of manifolds over $M$ with $f$ a homotopy equivalence the induced morphism $\mathcal{F}\left(N^{\prime}\right) \rightarrow \mathcal{F}(N)$ is an equivalence in $\mathcal{C}$.

Proof. It suffices to show that for a locally constant sheaf $\mathcal{F}$ on $M$ and a homotopy equivalence $f: N \rightarrow M$ the global sections $\mathcal{F}(M)$ and $f^{*} \mathcal{F}(N)$ are equivalent in $\mathcal{C}$. Thus we need to show that the diagram

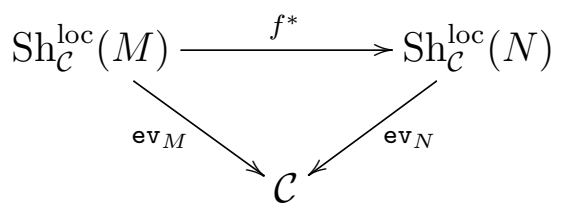

commutes. By the classification of locally constant sheaves as in Theorem B.2 we know that $f^{*}$ is an equivalence.

We now use that the global sections functors are right adjoint to the functors $\mathcal{C} \rightarrow$ $\operatorname{Sh}_{\mathcal{C}}^{\text {loc }}(M)$ and $\mathcal{C} \rightarrow \operatorname{Sh}_{\mathcal{C}}^{\text {loc }}(N)$ which are given by the inclusion of constant sheaves. Thus the commutativity of the diagram above is equivalent to the commutativity of the diagram of right adjoints. The right adjoint of $f^{*}$ is its inverse. But then we replace this inverse by $f^{*}$ we arrive at the point where we have to check that the diagram

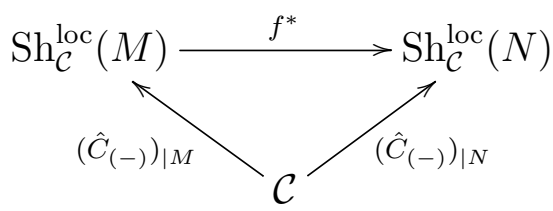


commutes, which is obvious.

\section{References}

[ABG10] M. Ando, A. J. Blumberg, and D. Gepner. Twists of $K$-theory and TMF. In Superstrings, geometry, topology, and $C^{*}$-algebras, volume 81 of Proc. Sympos. Pure Math., pages 27-63. Amer. Math. Soc., Providence, RI, 2010.

[ABG11] M. Ando, A. J. Blumberg, and D. Gepner. Parametrized spectra, multiplicative Thom spectra, and the twisted Umkehr map. ArXiv e-prints, December 2011.

[AG12] B. Antieau and D. Gepner. Brauer groups and étale cohomology in derived algebraic geometry. ArXiv e-prints, October 2012.

[AGG11] B. Antieau, D. Gepner, and J. M. Gómez. Actions of Eilenberg-MacLane spaces on K-theory spectra and uniqueness of twisted K-theory. ArXiv eprints, June 2011.

[AS04] M. Atiyah and G. Segal. Twisted K-theory. Ukr. Mat. Visn., 1(3):287-330, 2004.

[AS06] M. Atiyah and G. Segal. Twisted K-theory and cohomology. In Inspired by S. S. Chern, volume 11 of Nankai Tracts Math., pages 5-43. World Sci. Publ., Hackensack, NJ, 2006.

[BCM ${ }^{+}$02] P. Bouwknegt, A. L. Carey, V. Mathai, M. K. Murray, and D. Stevenson. Twisted $K$-theory and $K$-theory of bundle gerbes. Comm. Math. Phys., 228(1):17-45, 2002.

[BG13] U. Bunke and D. Gepner. Differential function spectra, the differential BeckerGottlieb transfer, and applications to differential algebraic K-theory. ArXiv e-prints, June 2013.

[BNV13] U. Bunke, T. Nikolaus, and M. Völkl. Differential cohomology theories as sheaves of spectra. ArXiv e-prints, December 2013.

[BS05] U. Bunke and T. Schick. On the topology of T-duality. Rev. Math. Phys., 17(1):77-112, 2005.

[BS09] U. Bunke and T. Schick. Smooth K-theory. Astérisque, (328):45-135 (2010), 2009 .

[Bun] U. Bunke. Differential cohomology. ArXiv e-prints, August 2012.

[BV13] N. Barcenas and M. Velasquez. Twisted equivariant K- Theory and KHomology of Sl3(Z). ArXiv e-prints, April 2013. 
[CMW09] A. L. Carey, J. Mickelsson, and B.-L. Wang. Differential twisted $K$-theory and applications. J. Geom. Phys., 59(5):632-653, 2009.

[DFM11] J. Distler, D.l S. Freed, and G. W. Moore. Orientifold précis. In Mathematical foundations of quantum field theory and perturbative string theory, volume 83 of Proc. Sympos. Pure Math., pages 159-172. Amer. Math. Soc., Providence, RI, 2011.

[DK70] P. Donovan and M. Karoubi. Graded Brauer groups and $K$-theory with local coefficients. Inst. Hautes Études Sci. Publ. Math., (38):5-25, 1970.

[FHT11a] D. S. Freed, M. J. Hopkins, and C. Teleman. Loop groups and twisted $K$ theory I. J. Topol., 4(4):737-798, 2011.

[FHT11b] D. S. Freed, M. J. Hopkins, and C. Teleman. Loop groups and twisted $K$ theory III. Ann. of Math. (2), 174(2):947-1007, 2011.

[FHT13] D. S. Freed, M. J. Hopkins, and C. Teleman. Loop groups and twisted $K$ theory II. J. Amer. Math. Soc., 26(3):595-644, 2013.

[Gom13] K. Gomi. Mickelsson's twisted K-theory invariant and its generalizations. ArXiv e-prints, March 2013.

[GT10] K. Gomi and Y. Terashima. Chern-Weil construction for twisted $K$-theory. Comm. Math. Phys., 299(1):225-254, 2010.

[Har12] A. J. Harju. Twisted K-theory constructions in the case of a decomposable Dixmier-Douady class II: Topological and equivariant models. ArXiv e-prints, November 2012.

[Hin97] V. Hinich. Homological algebra of homotopy algebras. Comm. Algebra, 25(10):3291-3323, 1997.

[HM12] A. J. Harju and J. Mickelsson. Twisted K-theory constructions in the case of a decomposable Dixmier-Douady class. ArXiv e-prints, August 2012.

[HMS94] M. J. Hopkins, M. Mahowald, and H. Sadofsky. Constructions of elements in Picard groups. In Topology and representation theory (Evanston, IL, 1992), volume 158 of Contemp. Math., pages 89-126. Amer. Math. Soc., Providence, RI, 1994.

[HS05] M. J. Hopkins and I. M. Singer. Quadratic functions in geometry, topology, and M-theory. J. Differential Geom., 70(3):329-452, 2005.

[Lur09] J. Lurie. Higher topos theory, volume 170 of Annals of Mathematics Studies. Princeton University Press, Princeton, NJ, 2009. 
[Lur11] J. Lurie. Higher algebra. Prepublication book draft. Available from http: //www.math.harvard.edu/ lurie/, 2011.

[MM65] J. W. Milnor and J. C. Moore. On the structure of Hopf algebras. Ann. of Math. (2), 81:211-264, 1965.

[MM13] A. Mathew and L. Meier. Affineness and chromatic homotopy theory. ArXiv e-prints, November 2013.

[MP07] J. Mickelsson and J.-P. Pellonpää. Families index theorem in supersymmetric WZW model and twisted $K$-theory: the $\mathrm{SU}(2)$ case. Comm. Math. Phys., 271(3):775-789, 2007.

[MS03] V. Mathai and D. Stevenson. Chern character in twisted $K$-theory: equivariant and holomorphic cases. Comm. Math. Phys., 236(1):161-186, 2003.

[MS06a] V. Mathai and D. Stevenson. On a generalized Connes-Hochschild-KostantRosenberg theorem. Adv. Math., 200(2):303-335, 2006.

[MS06b] J. P. May and J. Sigurdsson. Parametrized homotopy theory, volume 132 of Mathematical Surveys and Monographs. American Mathematical Society, Providence, RI, 2006.

[Sag11] S. Sagave. Spectra of units for periodic ring spectra and group completion of graded E-infinity spaces. ArXiv e-prints, November 2011.

[Shi07] B. Shipley. HZZ-algebra spectra are differential graded algebras. Amer. J. Math., 129(2):351-379, 2007.

[SS10] J. Simons and D. Sullivan. Structured vector bundles define differential $K$ theory. In Quanta of maths, volume 11 of Clay Math. Proc., pages 579-599. Amer. Math. Soc., Providence, RI, 2010.

[TX06] J.-L. Tu and P. Xu. Chern character for twisted $K$-theory of orbifolds. Adv. Math., 207(2):455-483, 2006.

[TXLG04] J.-L. Tu, P. Xu, and C. Laurent-Gengoux. Twisted $K$-theory of differentiable stacks. Ann. Sci. École Norm. Sup. (4), 37(6):841-910, 2004. 